\title{
1s2p RIXS Calculations for 3d Transition Metal Ions in Octahedral Symmetry
}

\author{
Patric Zimmermann $\mathbb{D}^{D}$, Myrtille O. J. Y. Hunault, and Frank M. F. de Groot $\mathbb{D}$
}

Debye Institute of Nanomaterial Science, Utrecht University, 3584 CA Utrecht, Netherlands

Correspondence should be addressed to Patric Zimmermann; p.zimmermann@uu.nl and Frank M. F. de Groot; F.M.F.deGroot@uu.nl

Received 11 November 2017; Accepted 27 December 2017; Published 24 June 2018

Academic Editor: Maria Carmen Yebra-Biurrun

Copyright ( 92018 Patric Zimmermann et al. This is an open access article distributed under the Creative Commons Attribution License, which permits unrestricted use, distribution, and reproduction in any medium, provided the original work is properly cited.

\begin{abstract}
We present a series of $1 \mathrm{~s} 2 \mathrm{p}$ resonant inelastic X-ray scattering (RIXS) calculations for $3 \mathrm{~d}$ transition metal ions in octahedral symmetry covering each ground state between $3 \mathrm{~d}^{0}$ and $3 \mathrm{~d}^{9}$. The calculations are performed in octahedral $\left(\mathrm{O}_{h}\right)$ symmetry using the crystal field multiplet theory. We discuss the crystal field effects and the selection rules with respect to the 1s2p RIXS pre-edge and compare their final state energies with the corresponding $2 \mathrm{p}$ X-ray absorption spectrum (XAS). The calculations provide a detailed understanding of 1s2p RIXS and serve as a basis for the future analysis of experimental spectra and also as a starting point for calculations that add additional channels including the nonlocal peaks.
\end{abstract}

\section{Introduction}

Throughout the past decades, X-ray absorption spectroscopy (XAS) as well as X-ray emission spectroscopy (XES) has played an important role towards the study of the electronic structure of transition metal complexes. The advent of synchrotron light sources has made high flux and highresolution measurements accessible to researchers worldwide. Especially, the second order resonant inelastic X-ray scattering (RIXS) process, where the X-ray emission spectra are measured as a function of the incident X-ray energy, has proven to be a valuable instrument with respect to the investigation of the electronic structure. Such resonant measurements are nowadays routinely performed at the synchrotrons around the world.

Here, we will focus on 1s2p RIXS measurements at the K preedge of the $3 \mathrm{~d}$ transition metals, where a $1 \mathrm{~s}$ core electron is promoted into the $3 \mathrm{~d}$ band (electric quadrupole 1s XAS) and the subsequent $\mathrm{K} \alpha_{1,2}$ decays (electric dipole $2 \mathrm{p}$ XES) are observed, which yields a two-dimensional RIXS map. In the following, such spectra will be referred to as $1 \mathrm{~s} 2 \mathrm{p}$ RIXS. In $2 \mathrm{p}$ XAS on the other hand, an electron is promoted from the $2 \mathrm{p}$ shell via a dipole transition into the $3 \mathrm{~d}$ level. Both types of spectra, the two-dimensional 1s2p RIXS (1s XAS, $2 p$ XES) and the one-dimensional $2 p$ XAS, are illustrated in Figure 1.

We limit our discussion of 1s2p RIXS to the pre-edge excitonic states, and we will not discuss the 1s2p RIXS plane related to excitations at the main edge and at higher energies. The 1s2p RIXS plane related to excitations at the edge can be explained from a convolution of the 1s XAS spectral shape and the $1 \mathrm{~s} 2 \mathrm{p}$ nonresonant XES spectral shape $[1,2]$.

Most transition metal compounds show a pre-edge structure in K-edge absorption (1s XAS) which relates to the local and nonlocal electronic structure as well as the symmetry of the system under study. Usually, the shape of the pre-edge is associated with the quadrupole transitions from the $1 \mathrm{~s}$ shell into the local $3 \mathrm{~d}$ orbitals.

In the case of inversion symmetry, local $4 \mathrm{p} 3 \mathrm{~d}$ mixing is forbidden by symmetry, but a nonlocal mixing of the local $4 \mathrm{p}$ orbitals with the $3 \mathrm{~d}$ orbitals of the neighbouring ligands $(\rightarrow$ nonlocal $)$ can alter the pre-edge structure and produce additionally the so-called nonlocal peaks [3-8] (e.g., $\mathrm{TiO}_{2}$ [3] and $\left.\mathrm{CrO}_{2}[9]\right)$. 


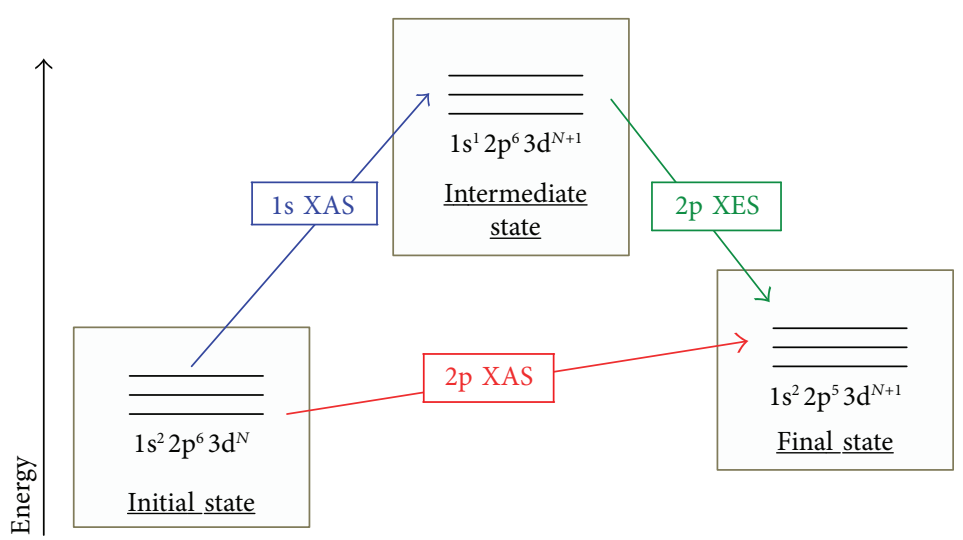

(a)

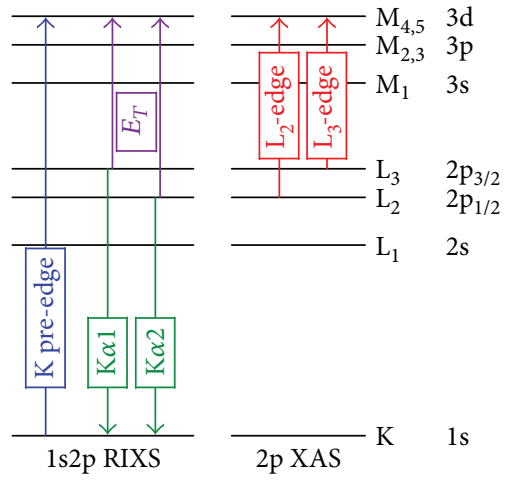

(b)

FIGURE 1: Scheme (a) illustrates the different pathways in the two-step 1s2p RIXS process, composed of the 1s XAS (K pre-edge) and 2p XES (K $\alpha$ decays) transitions and the direct $2 \mathrm{p}$ XAS $\left(\mathrm{L}_{2,3}\right.$-edge). The term scheme (b) illustrates the analogy between the energy transfer $E_{T}$ in 1 s2p RIXS and $2 \mathrm{p}$ XAS.

When there is no inversion symmetry, the quadrupole $(1 \mathrm{~s} \rightarrow 3 \mathrm{~d})$ and dipole $(1 \mathrm{~s} \rightarrow 4 \mathrm{p})$ peaks can mix locally in the pre-edge leading to an additional dipole contribution to the quadrupole peaks. For example, distortions or vibrational excitations can break the local inversion symmetry of $\mathrm{O}_{h}$ leading to some local dipole character in the pre-edge [10-12]. In addition, the tail of the much stronger dipole character of the main edge $(1 s \rightarrow 4 p)$ overlaps, but the X-ray emission due to this tail absorption can be subtracted from the 1s2p RIXS spectra. Due to this, the exact quadrupole and dipole contributions in the K pre-edge can be difficult to distinguish in experimental spectra. An exact analysis of such cases would go beyond the scope of the present paper, but it will be mentioned when relevant.

The motivation for 1s2p RIXS has its origin mainly in two aspects:

(1) High-resolution wavelength-dispersive detection of the $\mathrm{K} \alpha$ emission enables for the so-called high-energy resolution fluorescence-detected (HERFD) spectra. The advantage here is the effective suppression of the core-hole lifetime broadening due to the twostep $1 \mathrm{~s}$ XAS $\rightarrow 2 \mathrm{p}$ XES process.

(2) The final state configuration $1 \mathrm{~s}^{2} 2 \mathrm{p}^{5} 3 \mathrm{~d}^{N+1}$ is identical in $1 s 2 p$ RIXS and $2 p$ XAS, which enables to access " $\mathrm{L}_{2,3}$-edge information" with hard X-rays. However, it shall be noted that the different selection rules (quadrupole versus dipole) and the inclusion of an intermediate state in 1s2p RIXS can lead to significant differences in the spectra.

In this context, we are comparing the two-step 1s $2 \mathrm{p}$ RIXS process with the direct $2 \mathrm{p}$ XAS $\left(\mathrm{L}_{2,3}\right.$-edge) spectra for $3 \mathrm{~d}$ transition metal compounds. Based on the crystal field theory (CFT), we calculated at least one spectrum for each $3 \mathrm{~d}^{N}$ ground state, where $N \in\{0, \ldots, 9\}$. We aim to give an overview of the general structure of $1 \mathrm{~s} 2 \mathrm{p}$ RIXS for the $3 \mathrm{~d}^{N}$ transition metal series to illustrate their specific behaviour, but the calculated spectra shall also serve as a reference for future analysis.

In 1s2p RIXS, the 1s core hole created in 1s XAS interacts only weakly with the valence electrons. Since the 1 s shell has no orbital momentum $L$, there is no spin-orbit coupling with the other open shells. As we will see, this is important when comparing 1s2p RIXS with 2p XAS [13]. The consequences on the outcome of the spectra will be discussed throughout this paper.

The paper is organised as follows. In Section 2, we describe the theoretical background of the calculation of $1 s 2 p$ RIXS and the resulting selection rules. In Section 3, we give the computational details. In Section 4, we analyse the $1 s 2 p$ RIXS of three didactic cases in more detail, and in Section 5, we describe a series of the remaining $3 \mathrm{~d}^{N}$ cases including a short discussion of related experiments.

\section{Theory}

The necessary background being the theoretical framework and the computational tools used in this study are described in the following.

2.1. 1s2p RIXS with Kramers-Heisenberg. In 1s $2 p$ RIXS experiments, the incident energy is tuned around a resonance related to a $1 \mathrm{~s} \rightarrow 3 \mathrm{~d}$ transition (1s XAS). This excitation is followed by a subsequent radiative $2 p \rightarrow 1$ s decay ( $2 p$ XES). The emitted photons are detected with a wavelengthdispersive detector (crystal analyser) reaching in the hard $\mathrm{X}$-ray range sub-eV resolutions. Often this translates into a bandwidth of less than the lifetime broadening of the spectral features under study. This is often referred to as the RIXS sharpening effect overcoming the core-hole lifetime broadening which is related to the RIXS cross-section $[14,15]$.

The RIXS process is described as a two-step photonin photon-out scheme in which the incident photons undergo an inelastic scattering around a core resonance followed by a characteristic X-ray emission. The theoretical modelling of the 1s2p RIXS cross-section $\sigma$ is achieved with 
TABLE 1: Russell-Saunders term symbols for the $3 \mathrm{~d}^{N}$ free ion configurations giving the number of states, the corresponding number of energy levels, and the terms for the given configuration. Note that $d^{N}$ gives the same terms as $d^{10-N}$. A more detailed list can be found in Core Level Spectroscopy of Solids [13] (p. 103).

\begin{tabular}{lcccc}
\hline Config & States & Energy levels & Ground term & Excited terms \\
\hline $3 \mathrm{~d}^{1}, 3 \mathrm{~d}^{9}$ & 10 & 1 & ${ }^{2} \mathrm{D}$ & - \\
$3 \mathrm{~d}^{2}, 3 \mathrm{~d}^{8}$ & 45 & 5 & ${ }^{3} \mathrm{~F}$ & ${ }^{2} \mathrm{P},{ }^{1} \mathrm{G},{ }^{1} \mathrm{D},{ }^{1} \mathrm{~S}$ \\
$3 \mathrm{~d}^{3}, 3 \mathrm{~d}^{7}$ & 120 & 8 & ${ }^{4} \mathrm{~F}$ & ${ }^{4} \mathrm{P},{ }^{2} \mathrm{H},{ }^{2} \mathrm{G},{ }^{2} \mathrm{~F}, 2 \cdot{ }^{2} \mathrm{D},{ }^{2} \mathrm{P}$ \\
$3 \mathrm{~d}^{4}, 3 \mathrm{~d}^{6}$ & 210 & 16 & ${ }^{5} \mathrm{D}$ & ${ }^{6} \mathrm{H},{ }^{3} \mathrm{G}, 2 \cdot \cdot{ }^{3} \mathrm{~F},{ }^{3} \mathrm{D}, 2 \cdot{ }^{3} \mathrm{P},{ }^{1} \mathrm{I}, 2 \cdot{ }^{1} \mathrm{G},{ }^{1} \mathrm{~F}, 2 \cdot \cdot{ }^{1} \mathrm{D}, 2 \cdot \cdot{ }^{1} \mathrm{~S}$ \\
$3 \mathrm{~d}^{5}$ & 252 & 16 & ${ }^{4} \mathrm{G},{ }^{4} \mathrm{~F},{ }^{4} \mathrm{D},{ }^{4} \mathrm{P},{ }^{2} \mathrm{I},{ }^{2} \mathrm{H}, 2 \cdot{ }^{2} \mathrm{G}, 2 \cdot{ }^{2} \mathrm{~F}, 3 \cdot{ }^{2} \mathrm{D},{ }^{2} \mathrm{P},{ }^{2} \mathrm{~S}$ \\
\hline
\end{tabular}

the Kramers-Heisenberg formula for the second order quantum process which includes the 1 s XAS, $2 \mathrm{p}$ XES, and interference effects [14-19]:

$$
\begin{aligned}
\frac{\mathrm{d}^{2} \sigma}{\mathrm{d} \Omega \mathrm{d} \omega}= & \sum_{|f\rangle}\left|\sum_{|n\rangle} \frac{\left\langle f\left|\widehat{T}_{2}\right| n\right\rangle\left\langle n\left|\widehat{T}_{1}\right| i\right\rangle}{E_{i}-E_{n}+\hbar \Omega+i\left(\gamma_{n} / 2\right)}\right|^{2} \\
& \times \frac{\gamma_{f} / 2 \pi}{\left(E_{i}-E_{f}+\hbar \Omega-\hbar \omega\right)^{2}+\gamma_{f}^{2} / 4},
\end{aligned}
$$

with incident photons of energy $E_{\text {in }}=\hbar \Omega$, the inelastically scattered photons of energy $E_{\text {out }}=\hbar \omega$, and the energy transfer $E_{T}=\hbar \Omega-\hbar \omega$. The two-step process relates to the two transition operators, $\widehat{T}_{1}$ for the quadrupole $1 \mathrm{~s}$ XAS and $\widehat{T}_{2}$ for the dipole $2 p$ XES, describing the $1 \mathrm{~s} 2 \mathrm{p}$ RIXS cross-section $\sigma$ as the transition from the initial state $|i\rangle$ into the final states $|f\rangle$ via all possible intermediate states $|n\rangle$ of the system with energies $E_{i}, E_{f}$, and $E_{n}$, respectively. And finally, $\gamma_{n}$ and $\gamma_{f}$ are the natural line widths of the intermediate and final states. This means that the $1 \mathrm{~s}$ XAS is naturally broadened by the intermediate state lifetime $\gamma_{n}$ and $2 \mathrm{p}$ XES is naturally broadened by the final state lifetime $\gamma_{f}[15,20]$.

2.2. Crystal Field Theory: Local Model. We recapitulate briefly the aspects of the framework of the crystal field theory that are important for the present paper. Further details can be found in other references $[13,21]$. The initial, intermediate, and final states $|i\rangle,|n\rangle$, and $|f\rangle$, respectively, are described each by the corresponding Hamiltonian $\mathscr{H}$ :

$$
\mathscr{H}=\mathscr{H}_{\mathrm{kin}}+\mathscr{H}_{e / N}+\mathscr{H}_{\text {ele }}+\mathscr{H}_{\mathrm{SO}}+\mathscr{H}_{\mathrm{CF}} \text {, }
$$

where $\mathscr{H}_{\text {kin }}=\sum_{N} p_{i}^{2} / 2 m$ is the kinetic energy of the electrons with the momentum $p$ and mass $m, \mathscr{H}_{e / N}=\sum_{N}-Z e^{2} / r_{i}$ is the electrostatic interaction of the electron $i$ of charge $e$ with the nucleus at radius $r_{i}$ and charge $Z, \mathscr{H}_{e / e}=\sum_{\text {pairs }} e^{2} / r_{i j}$ is the electron-electron Coulomb interaction which is determined by the direct Coulomb repulsion and the Coulomb exchange interaction described by the Slater integrals $F^{k}$ and $G^{k}$, respectively, and $H_{\mathrm{SO}}=\sum_{N} \zeta\left(r_{i}\right) l_{i} \cdot s_{i}$ is the spin-orbit interaction on every open shell. These first four terms, together with a given ground state $|\psi\rangle$, describe the isolated atom in each state [21].

The electron-electron interactions of an electronic configuration give rise to multiple term symbols. The term symbol indicates a total orbital moment $\mathscr{L}$, total spin moment $\mathcal{S}$, and total angular moment $\mathcal{J}$, with $|\mathscr{L}-\mathcal{S}| \leq$ $\mathscr{J} \leq|\mathscr{L}+\mathcal{S}|$. This is the $\mathscr{L} . \mathcal{S}$ coupling scheme or RussellSaunders coupling, which will be used throughout the present paper (Table 1). In the absence of spin-orbit coupling, all terms with the same $\mathscr{L}$ and $\mathcal{S}$ have the same energy, giving an energy level that is $(2 \mathscr{L}+1)(2 \mathcal{S}+1)$-fold degenerate. When spin-orbit coupling is included, $\mathscr{L}$ and $\mathcal{S}$ lose their meaning and the terms are split in energy according to their $\mathscr{J}$ value, each with a degeneracy of $2 \mathscr{J}+1$. One can assign to these states a so-called Russel-Saunders term symbol ${ }^{2 \delta+1} X_{\mathscr{J}}$, where $X$ is representing the orbital momentum $\mathscr{L}$; for values of $0,1,2$, and 3 , one writes $\mathrm{S}, \mathrm{P}, \mathrm{D}$, and $\mathrm{F}$, respectively [21, 22]. For example, a single electron in an $s$ shell is given as ${ }^{2} S_{1 / 2}$, and a single electron in a $\mathrm{p}$ shell is represented as ${ }^{2} \mathrm{P}_{1 / 2}$ and ${ }^{2} \mathrm{P}_{3 / 2}$.

The term symbol describes the symmetry aspects, but it does not say anything about its relative energy [14]. The electron-electron repulsion and the spin-orbit coupling define the relative energy of the different terms within a configuration. Here, Hund's rules offer a convenient way to determine the state lowest in energy $(\max \mathcal{S}, \max \mathscr{L}$, and $\max \mathscr{J}$ if more than $1 / 2$ is filled, otherwise $\min \mathscr{J}$ ) [21].

The total number of states per configuration is calculated via the binomial coefficients [21]:

$$
\begin{aligned}
& 3 \mathrm{~d}^{N}: \quad\left(\begin{array}{l}
10 \\
N
\end{array}\right)=\frac{10 !}{N ! \cdot(10-N) !}, \\
& 1 \mathrm{~s}^{1} 3 \mathrm{~d}^{N+1}:\left(\begin{array}{l}
2 \\
1
\end{array}\right) \cdot\left(\begin{array}{c}
10 \\
N+1
\end{array}\right)=2 \cdot \frac{10 !}{(N+1) ! \cdot(10-(N+1)) !}, \\
& 2 \mathrm{p}^{5} 3 \mathrm{~d}^{N+1}:\left(\begin{array}{l}
6 \\
1
\end{array}\right) \cdot\left(\begin{array}{c}
10 \\
N+1
\end{array}\right)=6 \cdot \frac{10 !}{(N+1) ! \cdot(10-(N+1)) !} .
\end{aligned}
$$

The crystal field multiplet Hamiltonian $\mathscr{H}_{\mathrm{CF}}$ extends the atomic Hamiltonian with an electrostatic field created by the neighbouring atoms in the solid state. Because a large range of systems consist of a transition metal ion surrounded by six neighbouring atoms, where these neighbours are positioned at each corner of an octahedron centred around the transition metal atom, we will focus on the case of the cubic crystal field. The neighbours form a so-called octahedral field, which belongs to the $\mathrm{O}_{h}$ point group. Thus, we discuss all ions throughout this paper in 
TABle 2: Branching of the $\mathcal{S}, \mathscr{L}$, and $\mathscr{J}$ atomic terms in an octahedral crystal field $\left(\mathrm{O}_{3} \rightarrow \mathrm{O}_{h}\right)$ [28] (p.262). The first columns can be either the orbital momentum, the total spin $\mathcal{S}$, or the total angular momentum $\mathcal{F}$. The $g$ for gerade and the $u$ for ungerade must be added to indicate the parity.

\begin{tabular}{llc}
\hline $\mathrm{O}_{3}$, or $\mathcal{J}$ & $\rightarrow$ & $\mathrm{O}_{h}$ \\
$\mathcal{S}, \mathscr{L}$, & Term symbol \\
\hline $0(\mathrm{~S})$ & $\rightarrow$ & $\mathrm{A}_{1}$ \\
$1(\mathrm{P})$ & $\rightarrow$ & $\mathrm{T}_{1}$ \\
$2(\mathrm{D})$ & $\rightarrow$ & $\mathrm{E} \oplus \mathrm{T}_{2}$ \\
$3(\mathrm{~F})$ & $\rightarrow$ & $\mathrm{T}_{1} \oplus \mathrm{T}_{2} \oplus \mathrm{A}_{2}$ \\
$4(\mathrm{G})$ & $\rightarrow$ & $\mathrm{A}_{1} \oplus \mathrm{E} \oplus \mathrm{T}_{1} \oplus \mathrm{T}_{2}$ \\
$5(\mathrm{H})$ & $\rightarrow$ & $\mathrm{E} \oplus 2 \mathrm{~T}_{2} \oplus \mathrm{T}_{2}$ \\
$6(\mathrm{I})$ & $\rightarrow$ & $\mathrm{A}_{1} \oplus \mathrm{T}_{1} \oplus \mathrm{E} \oplus 2 \mathrm{~T}_{2} \oplus \mathrm{A}_{2}$ \\
7 & $\rightarrow$ & $2 \mathrm{~T}_{2} \oplus \mathrm{E} \oplus 2 \mathrm{~T}_{2} \oplus \mathrm{A}_{2}$ \\
8 & $\rightarrow$ & $\mathrm{A}_{1} \oplus 2 \mathrm{~T}_{1} \oplus 2 \mathrm{E} \oplus 2 \mathrm{~T}_{2}$ \\
$1 / 2$ & $\rightarrow$ & $\mathrm{E}_{1 / 2}$ \\
$3 / 4$ & $\rightarrow$ & $\mathrm{F}_{3 / 2}$ \\
$5 / 2$ & $\rightarrow$ & $\mathrm{F}_{3 / 2} \oplus \mathrm{E}_{5 / 2}$ \\
$7 / 2$ & $\rightarrow$ & $\mathrm{E}_{1 / 2} \oplus \mathrm{F}_{3 / 2} \oplus \mathrm{E}_{5 / 2}$ \\
$9 / 2$ & $\rightarrow$ & $\mathrm{E}_{1 / 2} \oplus 2 \mathrm{~F}_{3 / 2}$ \\
$11 / 2$ & $\rightarrow$ & $\mathrm{E}_{1 / 2} \oplus 2 \mathrm{~F}_{3 / 2} \oplus \mathrm{E}_{5 / 2}$ \\
$13 / 2$ & $\rightarrow$ & $\mathrm{E}_{1 / 2} \oplus 2 \mathrm{~F}_{3 / 2} \oplus 2 \mathrm{E}_{5 / 2}$ \\
$15 / 2$ & $\rightarrow$ & $\mathrm{E}_{1 / 2} \oplus 3 \mathrm{~F}_{3 / 2} \oplus \mathrm{E}_{5 / 2}$ \\
\hline
\end{tabular}

an octahedral symmetry $\left(\mathrm{O}_{h}\right)$. We are aware that this is not necessarily true for all $3 \mathrm{~d}$ transitions metal ions (e.g., $\mathrm{CrO}_{2}\left(\mathrm{Cr}^{4+}\right)$ and $\mathrm{TiO}_{2}\left(\mathrm{Ti}^{4+}\right)$ have tetragonal $\left(\mathrm{D}_{4 h}\right)$ symmetry; $\mathrm{Fe}_{2} \mathrm{O}_{3}\left(\mathrm{Fe}^{3+}\right)$ has $\mathrm{C}_{3}$ symmetry). However, we make this simplification since we are aiming to present and discuss the elementary differences for each case in an analogue and comparable way.

In a single-electron picture, in a spherical environment, the $3 \mathrm{~d}$ orbitals are degenerate. In $\mathrm{O}_{h}$, the cubic crystal field induces the splitting of the five $3 \mathrm{~d}$ orbitals into two symmetrically different groups: (1) the 3 -fold $t_{2 g}$ orbitals $(x y, x z$, and $y z)$, which point in between the six metal-ligand bonds and thus participate in $\pi$ bondings with the surrounding ligands, and (2) the 2-fold $e_{g}$ orbitals $\left(z^{2}\right.$ and $\left.x^{2}-y^{2}\right)$, which point along the metal-ligand bonds and therefore relate to $\sigma$ bondings. The splitting between the $e_{g}$ and $t_{2 g}$ orbitals is defined by the strength of the crystal field and is described by the parameter $10 \mathrm{D} q[13]$.

The symmetry of the multielectronic term changes from spherical symmetry $\left(\mathrm{O}_{3}\right)$ to octahedral symmetry $\left(\mathrm{O}_{h}\right)$ causing the $\mathscr{L}$ term (S, P, D, etc.) to branch to an $\mathrm{O}_{h}$ irreducible representation (IRREP): $\mathrm{S}\left(\mathrm{O}_{3}\right)$ branches into an $\mathrm{A}_{1}\left(\mathrm{O}_{h}\right)$ symmetry state, $\mathrm{P}\left(\mathrm{O}_{3}\right)$ branches into a $\mathrm{T}_{1}\left(\mathrm{O}_{h}\right)$ symmetry state, and $\mathrm{D}\left(\mathrm{O}_{3}\right)$ branches into $\mathrm{T}_{2}\left(\mathrm{O}_{h}\right)$ and $\mathrm{E}\left(\mathrm{O}_{h}\right)$ symmetry states. Because the point group $\mathrm{O}_{3}$ (resp., $\mathrm{O}_{h}$ ) contains the inversion, a parity information should be added for gerade or ungerade to the term, + or - for the atomic (spherical) terms, and $\mathrm{g}$ or $\mathrm{u}$ for the crystal field terms. The $3 \mathrm{~d}^{N}$ initial state and the intermediate state $1 \mathrm{~s}^{1} 3 \mathrm{~d}^{N+1}$ in $1 \mathrm{~s} 2 \mathrm{p}$ RIXS are gerade $(\mathrm{g})$, and the final state $2 \mathrm{p}^{5} 3 \mathrm{~d}^{N+1}$ is, due to the open $2 p$ shell, ungerade. In the following, the parity of the spherical term will be omitted for simplicity. All the branchings are given in Table 2, which we will use extensively throughout this paper.

The relative energies of the $\mathrm{O}_{h}$ IRREPs are calculated by adding the effect of the cubic crystal field $10 \mathrm{D} q$ to the atomic state energies. The diagrams of the relative energies with respect to the cubic crystal field, often without spin-orbit coupling, are known as Tanabe-Sugano diagrams. More generally, we will call them energy level diagram (ELD). They will appear in the context of the quadrupole 1s XAS because the ELD for any intermediate state in 1s2p RIXS with a $1 \mathrm{~s}^{1} 3 \mathrm{~d}^{N+1}$ electron configuration is the same as the diagram for a $1 \mathrm{~s}^{2} 3 \mathrm{~d}^{N+1}$ configuration times the ${ }^{2} \mathrm{~A}_{2 g}$ term to include the 1 s core hole.

When spin-orbit coupling (SOC) and crystal field are both to be included, there are in principle two ways to derive the term symbols: (1) by first deriving the branching of the uncoupled atomic terms (with $\mathscr{L}$ and $\mathcal{S}$ ) in $\mathrm{O}_{h}$ symmetry and then by deriving the spin-orbital coupling or (2) by first deriving the atomic $\mathscr{J}$ quantum number in the $\mathscr{L} . \mathcal{S}$ coupling scheme and then deriving the branching of the $\mathscr{J}$ value in the $\mathrm{O}_{h}$ point group. Both methods yield identical final results and the identical ground state for a given Hamiltonian (see, e.g., Figure 2).

The order in which the derivation should be done is related to the relative weights of the spin-orbit coupling and crystal field terms in the Hamiltonian. In the initial state of 1 s 2 p RIXS, the $3 \mathrm{~d}$ spin-orbit coupling $\zeta_{3 \mathrm{~d}}$ is small (a few tens of $\mathrm{meV}$ ) compared to the usual values of crystal field splitting $10 \mathrm{D} q$ (a few eV). As a result, in cases with neglectable spin-orbit interaction, the mixing between the spin and the orbital momenta is weak and thus $\mathscr{L}$ and $\mathcal{S}$ can still be used to describe the initial state. Similarly, for the intermediate state, the 1 s core hole with $\mathscr{L}=0$ does not have spin-orbit coupling. Therefore, in these cases, the $\mathscr{L} . \mathcal{S}$ coupling is achieved after the crystal field branching. (See Xu et al. [23] for details on $\mathscr{L} . \mathcal{S}$ coupling.)

In the present paper, we are focusing on the $3 \mathrm{~d}$ transition metal ions represented by a series of cases with a ground state electron configuration ranging from $3 \mathrm{~d}^{0}$ to $3 \mathrm{~d}^{9}$. Depending on the number of $3 \mathrm{~d}$ electrons, the value of the total spin $\mathcal{S}$ varies between 0 (minimum) and 5/2 (maximum). The branching for each value of $\mathcal{S}$ in $\mathrm{O}_{h}$ is given in Table 2. The $\mathscr{L}$.S -coupled term, also called $\mathscr{J}$ value IRREP, in $\mathrm{O}_{h}$ symmetry is obtained from the direct product of the $\mathscr{L}$ IRREP and the $\delta$ IRREP. For this calculation, the direct product tables Tables 3, 4, and 5 can be used. Note that the total orbital multiplicity is always maintained, for example,

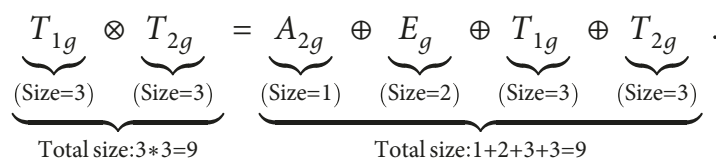

On the contrary, in the final state configuration, the strong spin-orbit coupling $\zeta_{2 \mathrm{p}}$ of the $2 \mathrm{~d}$ hole (a few $\mathrm{eV}$ ) 


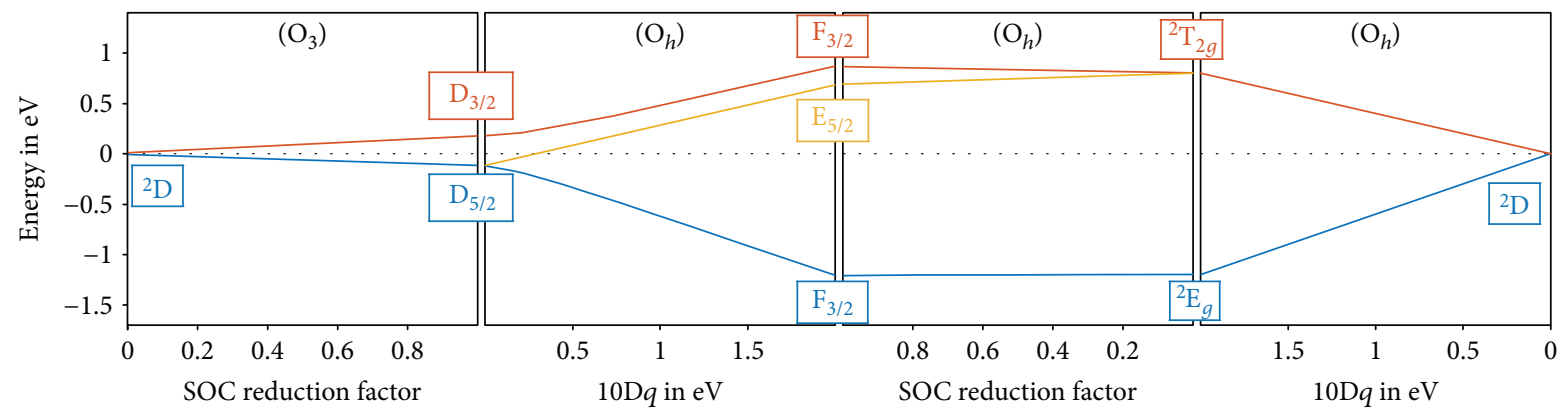

Figure 2: Initial state term schemes for $\mathrm{Cu}^{2+}\left(3 \mathrm{~d}^{9}\right)$ illustrating the effect of the $3 \mathrm{~d}$ SOC $\zeta_{3 \mathrm{~d}}$ and $\mathrm{O}_{h}$ crystal field $10 \mathrm{D} q$ and the resulting splittings of the atomic ground state term ${ }^{2} \mathrm{D}$.

TABLE 3: Direct products of representations (product table) in $\mathrm{O}_{h}$ symmetry.

\begin{tabular}{lccccc}
\hline $\mathrm{O}_{h}$ & $\mathrm{~A}_{1 g}$ & $\mathrm{~A}_{2 g}$ & $\mathrm{E}_{g}$ & $\mathrm{~T}_{1 g}$ & $\mathrm{~T}_{2 g}$ \\
\hline $\mathrm{A}_{1 g}$ & $\mathrm{~A}_{1 g}$ & $\mathrm{~A}_{2 g}$ & $\mathrm{E}_{g}$ & $\mathrm{~T}_{1 g}$ & $\mathrm{~T}_{2 g}$ \\
$\mathrm{~A}_{2 g}$ & & $\mathrm{~A}_{1 g}$ & $\mathrm{E}_{g}$ & $\mathrm{~T}_{2 g}$ & $\mathrm{~T}_{1 g}$ \\
$\mathrm{E}_{g}$ & & $\mathrm{~A}_{1 g} \oplus \mathrm{A}_{2 g} \oplus \mathrm{E}_{g}$ & $\mathrm{~T}_{1 g} \oplus \mathrm{T}_{2 g}$ & $\mathrm{~T}_{1 g} \oplus \mathrm{T}_{2 g}$ \\
$\mathrm{~T}_{1 g}$ & & & $\mathrm{~A}_{1 g} \oplus \mathrm{E}_{g} \oplus \mathrm{T}_{1 g} \oplus \mathrm{T}_{2 g}$ & $\mathrm{~A}_{2 g} \oplus \mathrm{E}_{g} \oplus \mathrm{T}_{1 g} \oplus \mathrm{T}_{2 g}$ \\
$\mathrm{~T}_{2 g}$ & & & & $\mathrm{~A}_{1 g} \oplus \mathrm{E}_{g} \oplus \mathrm{T}_{1 g} \oplus \mathrm{T}_{2 g}$ \\
\hline
\end{tabular}

TABLE 4: Direct products of representations (cont.) in $\mathrm{O}_{h}$ symmetry (dipole).

\begin{tabular}{lccccc}
\hline $\mathrm{O}_{h}$ & $\mathrm{~A}_{1 u}$ & $\mathrm{~A}_{2 u}$ & $\mathrm{E}_{u}$ & $\mathrm{~T}_{1 u}$ & $\mathrm{~T}_{2 u}$ \\
\hline $\mathrm{A}_{1 g}$ & $\mathrm{~A}_{1 u}$ & $\mathrm{~A}_{2 u}$ & $\mathrm{E}_{u}$ & $\mathrm{~T}_{1 u}$ & $\mathrm{~T}_{2 u}$ \\
$\mathrm{~A}_{2 g}$ & $\mathrm{~A}_{2 u}$ & $\mathrm{~A}_{1 u}$ & $\mathrm{E}_{u}$ & $\mathrm{~T}_{2 u}$ & $\mathrm{~T}_{1 u}$ \\
$\mathrm{E}_{g}$ & $\mathrm{E}_{u}$ & $\mathrm{E}_{u}$ & $\mathrm{~A}_{1 u} \oplus \mathrm{A}_{2 u} \oplus \mathrm{E}_{u}$ & $\mathrm{~T}_{1 u} \oplus \mathrm{T}_{2 u}$ & $\mathrm{~T}_{1 u} \oplus \mathrm{T}_{2 u}$ \\
$\mathrm{~T}_{1 g}$ & $\mathrm{~T}_{1 u}$ & $\mathrm{~T}_{2 u}$ & $\mathrm{~T}_{1 u} \oplus \mathrm{T}_{2 u}$ & $\mathrm{~A}_{1 u} \oplus \mathrm{E}_{u} \oplus \mathrm{T}_{1 u} \oplus \mathrm{T}_{2 u}$ & $\mathrm{~A}_{2 u} \oplus \mathrm{E}_{u} \oplus \mathrm{T}_{1 u} \oplus \mathrm{T}_{2 u}$ \\
$\mathrm{~T}_{2 g}$ & $\mathrm{~T}_{2 u}$ & $\mathrm{~T}_{1 u}$ & $\mathrm{~T}_{1 u} \oplus \mathrm{T}_{2 u}$ & $\mathrm{~A}_{2 u} \oplus \mathrm{E}_{u} \oplus \mathrm{T}_{1 u} \oplus \mathrm{T}_{2 u}$ & $\mathrm{~A}_{1 u} \oplus \mathrm{E}_{u} \oplus \mathrm{T}_{1 u} \oplus \mathrm{T}_{2 u}$ \\
\hline $\mathrm{A}_{1 u}$ & $\mathrm{~A}_{1 g}$ & $\mathrm{~A}_{2 g}$ & $\mathrm{E}_{g}$ & $\mathrm{~T}_{1 g}$ & $\mathrm{~T}_{2 g}$ \\
$\mathrm{~A}_{2 u}$ & & $\mathrm{~A}_{1 g}$ & $\mathrm{E}_{g}$ & $\mathrm{~T}_{1 g} \oplus \mathrm{T}_{2 g}$ & $\mathrm{~T}_{1 g} \oplus \mathrm{T}_{2 g}$ \\
$\mathrm{E}_{u}$ & & & $\mathrm{~A}_{1 g} \oplus \mathrm{E}_{g} \oplus \mathrm{T}_{1 g} \oplus \mathrm{T}_{2 g}$ & $\mathrm{~A}_{2 g} \oplus \mathrm{E}_{g} \oplus \mathrm{T}_{1 g} \oplus \mathrm{T}_{2 g}$ \\
$\mathrm{~T}_{1 u}$ & & & & $\mathrm{~A}_{1 g} \oplus \mathrm{E}_{g} \oplus \mathrm{T}_{1 g} \oplus \mathrm{T}_{2 g}$ \\
$\mathrm{~T}_{2 u}$ & & & &
\end{tabular}

TABLE 5: Direct products of representations (cont.) in $\mathrm{O}_{h}$ symmetry (SOC). Note that in this table, the parity (g: gerade; $u$ : ungerade) was omitted here as the table can be used for either case. For accurate labelling, the parity has to be added to the term symbol, where gerade times gerade equals gerade and gerade times ungerade equals an ungerade term symbol.

\begin{tabular}{lccc}
\hline $\mathrm{O}_{h}$ & $\mathrm{E}_{1 / 2}$ & $\mathrm{E}_{5 / 2}$ & $\mathrm{~F}_{3 / 2}$ \\
\hline $\mathrm{A}_{1}$ & $\mathrm{E}_{1 / 2}$ & $\mathrm{E}_{5 / 2}$ & $\mathrm{~F}_{3 / 2}$ \\
$\mathrm{~A}_{2}$ & $\mathrm{E}_{5 / 2}$ & $\mathrm{E}_{1 / 2}$ & $\mathrm{~F}_{3 / 2}$ \\
$\mathrm{E}$ & $\mathrm{F}_{3 / 2}$ & $\mathrm{~F}_{3 / 2}$ & $\mathrm{E}_{1 / 2} \oplus \mathrm{E}_{5 / 2} \oplus \mathrm{F}_{3 / 2}$ \\
$\mathrm{~T}_{1}$ & $\mathrm{E}_{1 / 2} \oplus \mathrm{F}_{3 / 2}$ & $\mathrm{E}_{1 / 2} \oplus \mathrm{F}_{3 / 2}$ & $\mathrm{E}_{1 / 2} \oplus \mathrm{E}_{5 / 2} \oplus 2 \mathrm{~F}_{3 / 2}$ \\
$\mathrm{~T}_{2}$ & $\mathrm{E}_{5 / 2} \oplus \mathrm{F}_{3 / 2}$ & $\mathrm{E}_{1 / 2} \oplus \mathrm{F}_{3 / 2}$ & $\mathrm{E}_{1 / 2} \oplus \mathrm{E}_{5 / 2} \oplus 2 \mathrm{~F}_{3 / 2}$ \\
\hline $\mathrm{E}_{1 / 2}$ & $\mathrm{~A}_{1} \oplus \mathrm{T}_{1}$ & $\mathrm{~A}_{2} \oplus \mathrm{T}_{2}$ & $\mathrm{E} \oplus \mathrm{T}_{1} \oplus \mathrm{T}_{2}$ \\
$\mathrm{E}_{5 / 2}$ & & $\mathrm{~A}_{1} \oplus \mathrm{T}_{1}$ & $\mathrm{E} \oplus \mathrm{T}_{1} \oplus \mathrm{T}_{2}$ \\
$\mathrm{~F}_{3 / 2}$ & & & $\mathrm{~A}_{1} \oplus \mathrm{A}_{2} \oplus \mathrm{E} \oplus 2 \mathrm{~T}_{1} \oplus \mathrm{T}_{2} \oplus \mathrm{T}_{2}$ \\
\hline
\end{tabular}

is dominant. This induces a strong mixing between the spin and orbital momenta and resulting in $\mathscr{L}$ and $\mathcal{S}$ not being good quantum numbers anymore. Instead, the total angular momentum $\mathcal{J}$ must be used to describe the final states. The effect of the $\mathrm{O}_{h}$ crystal field is added as the corresponding branching of the atomic $\mathscr{J}$ value IRREP. The branching for each value of $\mathcal{J}$ is also given in Table 2. The obtained IRREPs are either $\mathrm{A}_{1 u}, \mathrm{~T}_{1 u}, \mathrm{E}_{u}, \mathrm{~T}_{2 u}$, or $\mathrm{A}_{2 u}$ for integer $\mathcal{S}$ or $\mathscr{J}$. And they are $\mathrm{E}_{1 / 2 u}, \mathrm{~F}_{3 / 2 u}$, and $\mathrm{E}_{3 / 2 u}$ for half-integer values of $\mathcal{S}$ or $\mathcal{J}$.

As we will see across the $3 \mathrm{~d}^{N}$ series, the information of $\mathscr{L}$, $\mathcal{S}$, and $\mathscr{J}$ is important for the description of the ground state of the initial state electron configuration when comparing the one-step 2p XAS with the two-step 1s2p RIXS selection rules (see Section 2.3). Therefore, in an $\mathrm{O}_{h}$ crystal field, we will indicate the uncoupled atomic term (with $\mathscr{L}$ and $\delta$ ) along with the total angular momentum $\mathscr{J}$ value IRREP using the following notation: ${ }^{2 \delta+1} \mathscr{L}[\mathscr{J}]$. 
We illustrate this here with an example for $\mathrm{Ni}^{2+}\left(3 \mathrm{~d}^{8}\right)$ with the atomic ground state term ${ }^{3} \mathrm{~F}\left(\mathrm{O}_{3}\right)$.

$$
{ }^{3} \mathrm{~F} \stackrel{\mathrm{O}_{h}}{\longrightarrow}{ }^{3} \mathrm{~A}_{2 g}+{ }^{3} \mathrm{~T}_{1 g}+{ }^{3} \mathrm{~T}_{2 g} .
$$

Note that the spin multiplicity should be kept and is part of the term symbol because spin-orbit coupling is not yet included. The ${ }^{3} \mathrm{~A}_{2 g}$ symmetry represents in this case the state being the lowest in energy, and it is therefore the ground state term. In the next step, we derive the total angular momentum $\mathcal{J}$ IRREPs in the $\mathscr{L} \cdot \mathcal{S}$ coupling scheme $\left(\mathcal{S}=1 \rightarrow \otimes \mathrm{T}_{1 g}\right)$ for the three terms derived in (5):

${ }^{3} \mathrm{~A}_{2 g} \otimes \mathrm{T}_{1 g}=\mathrm{T}_{2 g} \quad$ (splitting of the $\mathrm{A}_{2 g}$ term due to $3 \mathrm{~d}$ SOC),

$$
\begin{array}{r}
{ }^{3} \mathrm{~T}_{1 g} \otimes \mathrm{T}_{1 g}=\mathrm{A}_{1 g}+\mathrm{E}_{g}+\mathrm{T}_{1 g}+\mathrm{T}_{2 g} \\
\text { (splitting of the } \left.\mathrm{T}_{1 g}^{3} \text { term due to } 3 \mathrm{~d} \mathrm{SOC}\right),
\end{array}
$$

$$
\begin{array}{r}
{ }^{3} \mathrm{~T}_{2 g} \otimes \mathrm{T}_{1 g}=\mathrm{A}_{2 g}+\mathrm{E}_{g}+\mathrm{T}_{1 g}+\mathrm{T}_{2 g} \\
\text { (splitting of the } \mathrm{e}_{2 g}^{3} \text { term due to 3d SOC). }
\end{array}
$$

In (6), we find the $\mathrm{T}_{2 g}$ total angular momentum IRREP as the ground state for the example $3 \mathrm{~d}^{8}$ in an octahedral crystal field. To summarise all this information in a condensed form, we write

$$
{ }^{3} \mathrm{~A}_{2 g}\left[\mathrm{~T}_{2 g}\right]
$$

This translates as a term without SOC with $\mathcal{S}$ and $\mathscr{L}$ [term with SOC using $\mathscr{J}$ ].

In other words, in (9), the term ${ }^{3} \mathrm{~A}_{2 g}$ is before the inclusion of SOC, where the spin multiplicity is 3 and the orbital momentum is given as $\mathrm{A}_{2 g}$. And the $\mathrm{T}_{2 g}$ term is the symmetry after spin-orbit interaction has been included (also identified as $\mathscr{J}$ value IRREP).

Finally, we note that charge transfer effects (e.g., the interaction with a $3 \mathrm{~d}^{N-1} \underline{L}$ configuration) are neglected but their relative importance will be discussed where appropriate throughout the $3 \mathrm{~d}^{N}$ series.

2.3. Selection Rules. The selection rules are the constrains that define the possibility of a transition between two states with a given transition operator. In other words, for 1s $2 p$ RIXS, the selection rules are the conditions for which the matrix elements $\left\langle f\left|\widehat{T}_{2}\right| n\right\rangle$ and $\left\langle n\left|\widehat{T}_{1}\right| i\right\rangle$ in the Kramers-Heisenberg equation (1) are nonzero. The operators $\widehat{T}_{1}$ and $\widehat{T}_{2}$ describe the interaction of the photon with matter. They are defined by the interaction Hamiltonian $\mathbf{p} \cdot \mathbf{A}$, where $\mathbf{p}$ is the momentum of the photon and $\mathbf{A}$ is the vector potential (e.g., the amplitude) of the electromagnetic field of the light. The electromagnetic interaction term is $(\varepsilon \cdot \mathbf{p}) e^{i \mathbf{k r}}$, where $\varepsilon$ is the polarisation of the photon and $\mathbf{k}$ is the propagation vector of the photon. In a multipole expansion, the $e^{i \mathbf{k r}}$ term can be decomposed in a Taylor series (the selection rules can be derived from the decomposition of the electron dipole/quadrupole transition matrix element into angular and radial parts using the Wigner-Eckart theorem $[13,24]$, where the triangular relations of the symbol determine the selection rules) which leads to the electric dipole and the electric quadrupole terms [25]. Further details can be found in Core Level Spectroscopy of Solids [13] and elsewhere [16, 24, 25].

Due to the electric dipole operator being the first-order term of this decomposition, the result is that the $\mathscr{J}$ quantum number can only change by a value of 0 or 1 . Thus, the electric dipole selection rule translates to $\Delta \mathscr{J}=+1,0$, or -1 . Because the electric quadrupole operator is the secondorder term of this decomposition, the resulting selection rule translates to $\Delta \mathscr{J}=-2,-1,0,+1$, or +2 .

However, when the spin-orbit coupling is neglected (or weak), the orbital momentum $\mathscr{L}$ and the spin momentum $\mathcal{S}$ can still be considered good quantum numbers. In this case, the transitions are spin-conserving $(\Delta \mathcal{S}=0)$ and only $\mathscr{L}$ changes. Due to the fact that light carries an orbital momentum of $|\mathscr{L}|=1$, this implies for electric dipole transitions $\Delta \mathscr{L}=+1$ or -1 . For the second-order term, the electric quadrupole transitions, this implies $\Delta \mathscr{L}=+2,0$, or -2 [21].

As a result, the transition operator $\widehat{T}_{1}$ of the $1 \mathrm{~s}$ XAS absorption step promoting an electron from 1 s to $3 \mathrm{~d}$ $(\Delta \mathscr{L}=+2)$ is an electric quadrupole term, and this step is forbidden as an electric dipole transition. On the contrary, the transition operator $\widehat{T}_{2}$ for the $2 \mathrm{p}$ XES decay from $2 \mathrm{p}$ to 1s $(\Delta \mathscr{L}=-1)$ is an electric dipole term. Similarly, the transition operator $\widehat{T}$ of the $2 \mathrm{p}$ XAS absorption step from $2 \mathrm{p}$ to 3d $(\Delta \mathscr{L}=+1)$ is also an electric dipole term.

These selection rules, defined in spherical symmetry, translate into the point group symmetry of the absorbing ion. In cubic symmetry $\left(\mathrm{O}_{h}\right)$, the selection rules of each operator depend on its symmetry properties. The electric dipole operator (first-rank tensor) behaves as the $\mathscr{J}=1 \operatorname{IRREP}\left(\mathrm{O}_{3}\right)$ and branches into $\mathrm{T}_{1 u}$ in $\mathrm{O}_{h}$. The electric quadrupole operator (second-rank tensor) behaves as a $\mathscr{J}=2$ IRREP $\left(\mathrm{O}_{3}\right)$ and branches in $\mathrm{O}_{h}$ into $\mathrm{T}_{2 g} \oplus \mathrm{E}_{g}$ (see Table 2). The transition matrix element between two states $|i\rangle,|f\rangle$ with the IRREPs $\Gamma_{i}$ and $\Gamma_{f}$ and the transition operator $\widehat{T}$ is nonzero if the direct product $\Gamma_{i} \otimes \Gamma_{\hat{T}}$ contains $\Gamma_{f}$, where $\Gamma_{\hat{T}}$ is the IRREP of the transition operator. In other words, the final state IRREPs $\Gamma_{f}$ accessible via the transition operator $\widehat{T}$ are given by the direct product $\Gamma_{i} \otimes \Gamma_{\hat{T}}$. The selection rules are derived from the direct product tables for the $\mathrm{O}_{h}$ point group (see Tables 3, 4, and 5) and give the symmetry of the accessible states.

The selection rules will be commented in more detail for each case of the $3 \mathrm{~d}^{N}$ series throughout this paper. Magnetic and natural dichroisms, which are a property from the crystal and not from the point group of the absorbing transition metal ion [26], will not be discussed here.

\section{Calculations and Computational Details}

All calculations are done using the framework of the crystal field multiplet theory, which is a multielectronic, semiempirical approach initially developed by Thole et al. [27] and further established by Butler and Cowan $[28,29]$. It takes into 
TABLE 6: Summary of the different notations in $\mathrm{O}_{h}$ symmetry. T/S/K: Tanabe/Sugano/Kamimura, though Sugano also uses a variation of this notation as shown. The CTM4XAS program uses mostly the Butler notation, though the ora-file translation is listed for ease of use.

\begin{tabular}{lcccccc}
\hline Altmann & Mulliken & $\mathrm{T} / \mathrm{S} / \mathrm{K}$ & Sugano & Koster/Bethe & Butler & CTM4XAS \\
\hline $\mathrm{A}_{1}$ & $\mathrm{~A}_{1}$ & $\mathrm{~A}_{1}$ & $\mathrm{~A}_{1}$ & $\Gamma_{1}$ & 0 & 0 \\
$\mathrm{~A}_{2}$ & $\mathrm{~A}_{2}$ & $\mathrm{~A}_{2}$ & $\mathrm{~A}_{2}$ & $\Gamma_{2}$ & $\tilde{0}$ & $\wedge$ \\
$\mathrm{E}$ & $\mathrm{E}$ & $\mathrm{E}$ & $\mathrm{E}$ & $\Gamma_{3}$ & 2 & 2 \\
$\mathrm{~T}_{1}$ & $\mathrm{~T}_{1}$ & $\mathrm{~T}_{1}$ & $\mathrm{~T}_{1}$ & $\Gamma_{4}$ & 1 & 1 \\
$\mathrm{~T}_{2}$ & $\mathrm{~T}_{2}$ & $\mathrm{~T}_{2}$ & $\mathrm{~T}_{2}$ & $\Gamma_{5}$ & $\tilde{1}$ & $\wedge 1$ \\
\hline $\mathrm{E}_{1 / 2}$ & $\mathrm{E}^{\prime}$ & $\mathrm{E}$ or $\mathrm{E}_{1}$ & $\mathrm{E}_{1 / 2}$ & $\Gamma_{6}$ & $1 / 2$ & $\mathrm{SO}$ \\
$\mathrm{F}_{3 / 2}$ & $\mathrm{U}$ & $\mathrm{G}$ & $\mathrm{G}_{3 / 2}$ & $\Gamma_{8}$ & $3 / 2$ & $\mathrm{~S} 1$ \\
$\mathrm{E}_{5 / 2}$ & $\mathrm{E}^{\prime \prime}$ & $\mathrm{E}^{\prime}$ or $\mathrm{E}_{2}{ }^{\prime \prime}$ & $\mathrm{E}_{5 / 2}$ & $\Gamma_{7}$ & $1 / 2$ & $\wedge \mathrm{S} 0$ \\
\hline
\end{tabular}

TABLE 7: Summary of the ground state terms, number of microstates, and crystal field values (10Dq) for all ions used in our calculations.

\begin{tabular}{|c|c|c|c|c|c|c|c|c|c|}
\hline \multirow{3}{*}{$\begin{array}{l}\text { GS } \\
3 \mathrm{~d}^{N}\end{array}$} & \multirow[t]{3}{*}{ Ion } & \multicolumn{3}{|c|}{ Ground state terms } & \multicolumn{2}{|c|}{$\begin{array}{c}\text { Number } \\
\text { of MS }\end{array}$} & \multirow{3}{*}{$\begin{array}{c}\text { Total GS } \\
(10)\end{array}$} & \multicolumn{2}{|c|}{$10 \mathrm{D} q$} \\
\hline & & $\mathrm{O}_{3}$ & \multicolumn{2}{|c|}{$\mathrm{O}_{h}$} & & & & & \\
\hline & & HS & HS & LS & HS & LS & & HS & LS \\
\hline $3 d^{0}$ & $\mathrm{Ti}^{4+}$ & & ${ }^{1} \mathrm{~A}_{1 g}\left[\mathrm{~A}_{1 g}\right]$ & & & & & & \\
\hline $3 \mathrm{~d}^{1}$ & $\begin{array}{ll}11 \\
\mathrm{Ti}^{3+}\end{array}$ & ${ }^{1} \mathrm{~S}$ & ${ }^{2} \mathrm{~T}_{2 g}\left[\mathrm{~F}_{3 / 2 g}\right]$ & & 1 & & 1 & $0 \mathrm{eV} \& 2.1 \mathrm{eV}$ & \\
\hline $3 d^{2}$ & $\mathrm{Cr}^{4+}$ & $\begin{array}{l}{ }^{2} \mathrm{D} \\
{ }^{3} \mathrm{~F}\end{array}$ & ${ }^{3} \mathrm{~T}_{1 q}\left[\mathrm{E}_{q}\right]$ & & 6 & & 10 & $1.5 \mathrm{eV}$ & \\
\hline $3 d^{3}$ & $\mathrm{Cr}^{3+}$ & ${ }^{\mathrm{F}}$ & ${ }^{4} \mathrm{~A}_{20}\left[\mathrm{~F}_{3 / 2}\right]$ & & 9 & & 45 & $2.1 \mathrm{eV}$ & \\
\hline $3 d^{4}$ & $\mathrm{Cr}^{3+}$ & ${ }^{2} \mathrm{~F}$ & $\mathrm{~A}_{2 g}\left[\mathrm{I}_{3 / 2 g}\right]$ & ${ }^{1}{ }_{1 g}\left[A_{1 g}\right]$ & 4 & 9 & 120 & $1.5 \mathrm{eV}$ & $3.9 \mathrm{eV}$ \\
\hline $3 d^{2}$ & $\mathrm{Mn}^{3+}$ & ${ }^{5} \mathrm{D}$ & ${ }^{5} \mathrm{E}_{g}\left[\mathrm{~A}_{1 g}\right]$ & ${ }^{2} \mathrm{~T}_{2 g}\left[\mathrm{E}_{5 / 2 g}\right]$ & 10 & 6 & 210 & $1.5 \mathrm{eV}$ & $3.9 \mathrm{eV}$ \\
\hline $3 d^{5}$ & $\mathrm{Fe}^{3+}$ & ${ }^{6} \mathrm{~S}$ & ${ }^{6} \mathrm{~A}_{1 g}\left[\mathrm{~F}_{3 / 2 g}\right]$ & ${ }^{1} \mathrm{~A}_{1 g}\left[\mathrm{~A}_{1 g}\right]$ & 6 & 1 & 252 & $1.5 \mathrm{eV}$ & $3.0 \mathrm{eV}$ \\
\hline $3 d^{6}$ & $\mathrm{Fe}^{2+}$ & ${ }^{5} \mathrm{D}$ & ${ }^{5} \mathrm{~T}_{2 q}\left[\mathrm{~T}_{2 q}\right]$ & ${ }^{2} \mathrm{E}_{q}\left[\mathrm{~F}_{3 / 2 q}\right]$ & 15 & 4 & 210 & $0.9 \mathrm{eV}$ & $3.0 \mathrm{eV}$ \\
\hline $3 d^{7}$ & $\mathrm{Co}^{2+}$ & ${ }^{4} \mathrm{~F}$ & ${ }^{4} \mathrm{~T}_{10}\left[\mathrm{E}_{1 / 2]}\right.$ & & 12 & & 120 & $0.9 \mathrm{eV}$ & \\
\hline $3 d^{8}$ & $\mathrm{Ni}^{2+}$ & ${ }^{3} \mathrm{~F}$ & ${ }^{1} 1 g\left[\mathrm{~L}_{1 / 2 g}\right]$ & & 3 & & 45 & $0.9 \mathrm{eV}$ & \\
\hline $3 d^{9}$ & $\mathrm{Cu}^{2+}$ & ${ }^{2} \mathrm{D}$ & $\begin{array}{c}\mathrm{A}_{2 g}\left[\mathrm{I}_{2 g}\right] \\
{ }^{2} \mathrm{E}_{g}\left[\mathrm{~F}_{3 / 2 g}\right]\end{array}$ & & 4 & & 10 & $0 \mathrm{eV} \& 3.0 \mathrm{eV}$ & \\
\hline
\end{tabular}

account all the $3 \mathrm{~d}-3 \mathrm{~d}, 1 \mathrm{~s}-3 \mathrm{~d}$, and $2 \mathrm{p}-3 \mathrm{~d}$ electronic Coulomb interactions, as well as the spin-orbit coupling $\zeta$ on every open shell of the absorbing atom (e.g., $2 \mathrm{p} \operatorname{SOC} \zeta_{2 \mathrm{p}}$ and $3 \mathrm{~d}$ SOC $\zeta_{3 \mathrm{~d}}$ in $1 \mathrm{~s} 2 \mathrm{p}$ RIXS).

The $2 p$ XAS and 1s $2 p$ RIXS spectra were calculated using Quanty which uses second quantisation and the Lanczos recursion method for the exact diagonalisation and Green functions to calculate the spectra [30-32]. This method enables to avoid the explicit calculation of the intermediate and final states, which are only defined with their respective Hamiltonian. Each of these many-body states is described by a linear combination of Slater determinants [31]. The atomic electronic interactions are parametrised by the Slater integrals, which are reduced to $80 \%$ of the Hartree-Fock values. The latter has empirically proven to correspond to the actual atomic values. For the spin-orbit coupling parameters $\zeta_{2 \mathrm{p}}, \zeta_{3 \mathrm{~d}}$, the Hartree-Fock calculated values are used. All calculations are done in octahedral symmetry $\left(\mathrm{O}_{h}\right)$, and the crystal field is defined by the crystal field parameter $10 \mathrm{D} q$. The same value is used for the initial, intermediate, and final states. The influence of the crystal field splitting is investigated by varying the parameter $10 \mathrm{D} q$ from $0 \mathrm{eV}$ up to a few $\mathrm{eV}$. The symmetry IRREPs of the labels of the final states in the $\mathrm{O}_{h}$ point group are obtained using the CTM4XAS program [33].
(Table 6 shows a general list of different notations to translate between them.) The natural broadenings $\gamma_{n}=0.5 \mathrm{eV}$ and $\gamma_{f}=0.25 \mathrm{eV}$ for the intermediate state and final state, respectively, were applied.

All RIXS intensities are given as calculated by Quanty. They reflect the total absorption as the sum over the five quadrupole basis components and the three dipole emission polarisations and thus imply an isotropic spectrum. When needed, one can in fact also compute the scattered photons as the percentage of the incident beam in dependency of a given experimental setup. The $2 \mathrm{p}$ XAS spectra are scaled for the best comparison for each case. The energies $E_{\text {in }}$ and $E_{\text {out }}$ are given by Quanty relative to the centre of gravity for each of the three states in 1s $2 p$ RIXS, being the $3 \mathrm{~d}^{N}, 1 \mathrm{~s}^{1} 3 \mathrm{~d}^{N+1}$, and $2 \mathrm{p}^{5} 3 \mathrm{~d}^{N+1}$ configurations, respectively. Thus, also the $E_{T}=0 \mathrm{eV}$ position of the energy transfer $E_{T}=E_{\text {in }}-E_{\text {out }}$ is based on the centre of gravity and $E_{T}$ does not reflect the real energy transfer.

More details on the method can be found elsewhere $[13,30-32,34]$ and the references therein. The calculations are performed for a temperature of $T=300 \mathrm{~K}$ as described in the following.

3.1. $T=300 \mathrm{~K}$ Approximation: Boltzmann Distribution. The multielectronic ground state at ambient condition is 


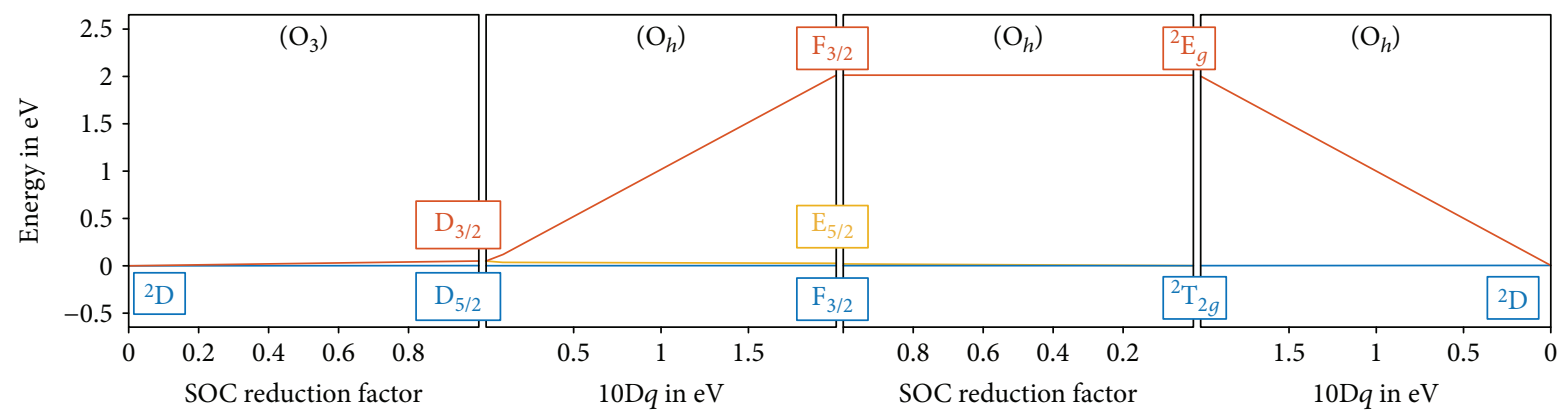

FIgURE 3: Initial state term schemes of $\mathrm{Ti}^{3+}\left(3 \mathrm{~d}^{1}\right)$ illustrating the effect of the $3 \mathrm{~d}$ SOC and an $\mathrm{O}_{h}$ crystal field 10D $q$ on the splittings of the atomic ground state term ${ }^{2} \mathrm{D}$.

a linear combination of an ensemble of $\mathscr{N}$ microstates $\psi_{i}$ with $i \in\{0, \ldots, \mathcal{N}-1\}$. In general, the Coulomb and spinorbit interactions as well as crystal field effects can induce a mixing of different states, leading to complex multielectronic states involving many microstates. For example, a $3 \mathrm{~d}^{5}$ configuration has in total 252 microstates, but only $\mathcal{N}=6$ microstates form in high spin the atomic multielectronic ground state ${ }^{6} \mathrm{~S}\left(\mathrm{O}_{3}\right)$ (Table 7 ).

However, at absolute zero $(T=0 \mathrm{~K})$, formally, only the state $\psi_{0}$ lowest in energy is populated. Thus, the calculated spectra result from a linear combination of each contributing state, weighted according to their respective population, because for temperatures $T>0 \mathrm{~K}$ higher states are also partially occupied. The population is described with the Boltzmann distribution.

It gives the population probability $p_{i}$ for each state $\psi_{i}$ in dependency of its relative energy $E_{i}-E_{0}$, with $E_{0}=E\left(\psi_{0}\right)$ being the energy of the lowest microstate $\psi_{0}$, and absolute temperature $T$. It is given (with Boltzmann constant $k_{B}$ ) as

$$
p_{i}=\frac{1}{a} \exp \left[-\frac{E_{i}-E_{0}}{k_{B} T}\right],
$$

with

$$
a=\sum_{l=0}^{\mathcal{N}} \exp \left[-\frac{E_{l}-E_{0}}{k_{B} T}\right]
$$

In the present case, the eigenvalues calculated with Quanty from $\mathscr{H}|\psi\rangle=E|\psi\rangle$ give the energies $E_{i}$ for each microstate $\psi_{i}$, which are used in (10) and (11) to compute the coefficients $p_{i}$ for the Boltzmann linear combination of each multielectronic ground state.

For example, $\mathrm{Ti}^{3+}\left(3 \mathrm{~d}^{1}\right)$ has the atomic ground state ${ }^{2} \mathrm{D}$ with $\mathcal{N}=10$ microstates. In other words, the single $3 \mathrm{~d}$ electron can be in 10 different states. In an $\mathrm{O}_{h}$ crystal field, this ground state branches without $3 \mathrm{~d}$ spin-orbit interaction into two crystal field terms: ${ }^{2} \mathrm{D} \stackrel{\mathrm{O}_{h}}{\rightarrow}{ }^{2} \mathrm{~T}_{2 g} \oplus^{2} \mathrm{E}_{g}$.

The term ${ }^{2} \mathrm{~T}_{2 g}$ represents the multielectronic ground state in an $\mathrm{O}_{h}$ crystal field, formed as a linear combination of $\mathcal{N}=6$ microstates. For $3 \mathrm{~d}^{1}$, the ${ }^{2} \mathrm{~T}_{2 g}$ term is the lowest in energy as can be seen in the single electronic picture: $\left|t_{2 g}^{1} e_{g}^{0}\right\rangle$, as opposed to the ${ }^{2} \mathrm{E}_{g}$ with $\left|t_{2 g}^{0} e_{g}^{1}\right\rangle$ in 4 possible microstates.
Furthermore, it should be noted that the $\mathcal{N}$ microstates forming the multielectronic ground state are a priori not degenerate. For $\mathrm{Ti}^{3+}$, the lowest 10 microstates are in fact partially degenerate. With $3 \mathrm{~d}$ spin-orbit interaction included, the microstates are spread in a $4: 2: 4$ ratio, corresponding to the terms $\mathrm{F}_{3 / 2}, \mathrm{E}_{5 / 2}$, and $\mathrm{F}_{3 / 2}$, respectively. In other words, the lowest four microstates $\psi_{0}, \psi_{1}, \psi_{2}$, and $\psi_{3}$ are degenerate in energy $\left(E_{0}=E_{1}=E_{2}=E_{3}\right)$, and the two states $\psi_{4}$ and $\psi_{5}$ are degenerate $\left(E_{4}=E_{5}\right)$, as well as the remaining 4 microstates $\psi_{6}, \psi_{7}, \psi_{8}$, and $\psi_{9}\left(E_{6}=E_{7}=E_{8}=E_{9}\right)$. Hence, the 10 microstates, which are related to the atomic multielectronic ground state ${ }^{2} \mathrm{D}\left(\mathrm{O}_{3}\right)$ and are split by the crystal field and spin-orbit interaction, are spread over three different energy levels. However, the six microstates forming the ${ }^{2} \mathrm{~T}_{2 g}\left(\mathrm{O}_{h}\right)$ term are only spread over two different energies (see Figure 3 ).

Note, if not stated otherwise, we only use the lowest crystal field ground state for the Boltzmann linear combination (e.g., ${ }^{2} \mathrm{~T}_{2 g}$ for $\mathrm{Ti}^{3+}$ ); the next multielectronic term higher in energy (e.g., ${ }^{2} \mathrm{E}_{g}$ for $\mathrm{Ti}^{3+}$ ) is neglected. This approximation relates to the fact that in general the second multielectronic term has a relatively small contribution in most cases.

\section{Didactic Examples}

In this section, we discuss three model systems as didactic examples in detail before the results for the remaining $3 \mathrm{~d}^{N}$ series (with $N \in\{1, \ldots, 7\}$ ) which are discussed in a more summarising manner (Section 5). Here, we will emphasise the elementary differences between the atomic $\left(\mathrm{O}_{3}\right)$ and the crystal field $\left(\mathrm{O}_{h}\right)$ cases to illustrate the effects with respect to the selection rules. Furthermore, we compare the results due to the different possible pathways in the direct one-step 2p XAS and the two-step 1s2p RIXS and the corresponding spectra.

As didactical examples, we use the following three cases:

(i) $3 \mathrm{~d}^{9} \mathrm{Cu}^{2+}$ : single open shell with only a single hole in each state

(ii) $3 \mathrm{~d}^{0} \mathrm{Ti}^{4+}$ : two peaks in the 1 XAS split by the crystal field $10 \mathrm{D} q$

(iii) $3 \mathrm{~d}^{8} \mathrm{Ni}^{2+}$ : a common model system often used in education 


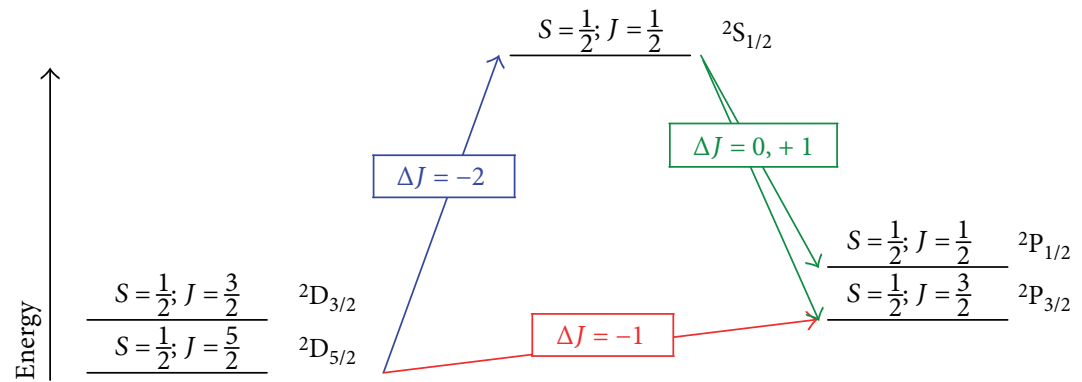

FIGURE 4: Atomic case: term scheme (with SOC; no crystal field) comparing the two-step (1s XAS, 2p XES) 1s2p RIXS and the one-step 2p XAS pathways for $\mathrm{Cu}^{2+}$. The sum over all $\mathscr{J}$ multiplicities $(2 \mathscr{F}+1)$ yields the total number of microstates per configuration.
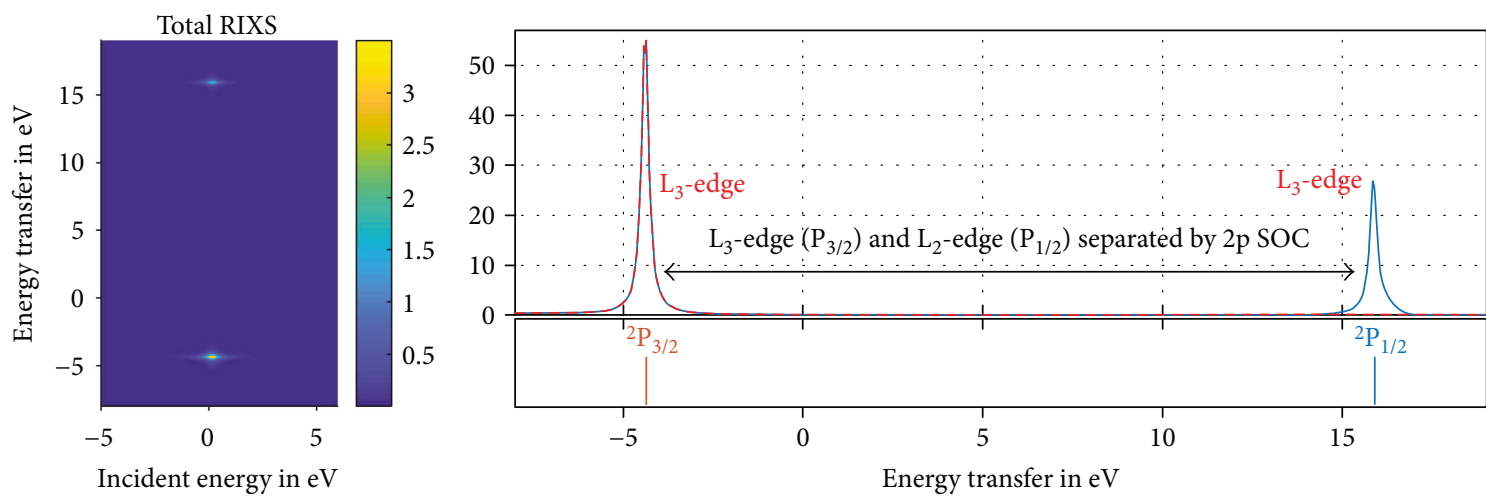

$$
\begin{aligned}
& \text { - IIE-RIXS } \\
& ---\mathrm{L}_{2,3} \text {-edge }
\end{aligned}
$$

FIGURE 5: Calculated atomic 1s2p RIXS map with $2 p$ XES projection for an isolated $\mathrm{Cu}^{2+}\left(3 \mathrm{~d}^{9}\right)$. Notice the $2 \mathrm{p}$ XAS $\left(\mathrm{L}_{2,3}\right.$-edge) (dashed red) has only one peak, because only the final state with $\mathcal{J}=3 / 2$ can be populated. See also Figure 7.3 in Core Level Spectroscopy of Solids [13].

We aim to assign the symmetry labels to the relevant peaks in the 1s2p RIXS spectra and compare the $2 p$ XES to the well-understood $\mathrm{L}_{2,3}$-edge (2p XAS) spectra [35].

4.1. $3 d^{9}$ : Divalent Copper $\mathrm{Cu}^{2+}$-Crystal Field Effects Altering the Selection Rules. In this part, we investigate the elementary differences between 1s 2 p RIXS and $2 p$ XAS for a divalent copper ion $\mathrm{Cu}^{2+}$ with a $3 \mathrm{~d}^{9}$ ground state. Each successive step of the 1s2p RIXS process has an electronic configuration with only one open shell and a single hole in the $3 \mathrm{~d}, 1 \mathrm{~s}$, and $2 \mathrm{p}$ shells, respectively.

$$
\begin{aligned}
\text { 1s2p RIXS : } 1 \mathrm{~s}^{2} 2 \mathrm{p}^{6} 3 \mathrm{~d}^{9} \frac{\Delta \mathscr{J}= \pm 2}{1 \mathrm{XXA}} 1 \mathrm{~s}^{1} 2 \mathrm{p}^{6} 3 \mathrm{~d}^{10} \stackrel{\Delta \mathscr{J}=0, \pm 1}{2 \mathrm{p} \mathrm{XES}} 1 \mathrm{~s}^{2} 2 \mathrm{p}^{5} 3 \mathrm{~d}^{10}, \\
\text { 2p XAS : } 1 \mathrm{~s}^{2} 2 \mathrm{p}^{6} 3 \mathrm{~d}^{9} \underset{\Delta \mathscr{J}=0, \pm 1}{\stackrel{2 \mathrm{pXAS}}{\longrightarrow}} 1 \mathrm{~s}^{2} 2 \mathrm{p}^{5} 3 \mathrm{~d}^{10} .
\end{aligned}
$$

We first describe the atomic case of an isolated ion without any crystal field. The electronic configuration of the initial state of $\mathrm{Cu}^{2+}$ is $1 \mathrm{~s}^{2} 2 \mathrm{p}^{6} 3 \mathrm{~d}^{9}$. In spherical symmetry $\left(\mathrm{O}_{3}\right)$, the $3 \mathrm{~d}$ spin-orbit coupling (SOC) $\zeta_{3 \mathrm{~d}}$ induces the splitting of the 10 -fold degenerate ground state ${ }^{2} \mathrm{D}$ (10 microstates) into two multielectronic states ${ }^{2} \mathrm{D}_{5 / 2}\left(\mathrm{O}_{3}\right)$ and ${ }^{2} \mathrm{D}_{3 / 2}\left(\mathrm{O}_{3}\right)$, defined by $\mathscr{J}=5 / 2$ (ground state) and $\mathcal{J}=3 / 2$, respectively. These two states are separated in energy by $\Delta E=5 / 2 \zeta_{3 \mathrm{~d}}$.
The electronic configuration of the $1 \mathrm{~s} 2 \mathrm{p}$ RIXS intermediate state of $\mathrm{Cu}^{2+}$ is $1 \mathrm{~s}^{1} 2 \mathrm{p}^{6} 3 \mathrm{~d}^{10}$, which corresponds to the 2fold degenerate state ${ }^{2} \mathrm{~S}_{1 / 2}\left(\mathrm{O}_{3}\right)$ with $\mathscr{J}=1 / 2$. The electronic configuration of the final state in $2 \mathrm{p}$ XAS and $1 s 2 \mathrm{p}$ RIXS of $\mathrm{Cu}^{2+}$ is $1 \mathrm{~s}^{2} 2 \mathrm{p}^{5} 3 \mathrm{~d}^{10}$, which splits due to the 2p spin-orbit coupling $\zeta_{2 p}$ into $\mathscr{J}=3 / 2$ (lowest energy state) and $\mathscr{J}=1 / 2$ separated by $3 / 2 \zeta_{2 \mathrm{p}}$. This is summarised in the scheme in Figure 4.

Since the ground state of the initial state is $\mathscr{J}=5 / 2$, the electric dipole absorption operator (2p XAS) enables only to reach the $\mathscr{J}=3 / 2$ final state $(\Delta J=-1)$. In 1 s2p RIXS, the selection rules of the electric quadrupole absorption for 1s XAS $(\Delta J=-2)$ enable to reach the intermediate state $\mathscr{J}=1 / 2$. The subsequent electric dipole emission (2p XES) enables to reach both $\mathscr{J}=1 / 2$ and $\mathscr{J}=3 / 2$ final states. As a result, for an isolated $\mathrm{Cu}^{2+}$ ion in spherical symmetry, the 2p XAS shows only one peak, while the 1s2p RIXS shows two peaks. The resulting spectra are shown in Figure 5.

In the vertical direction, the $\mathrm{K} \alpha_{1}$ and $\mathrm{K} \alpha_{2}$ decays (2p XES) appear as two separate peaks. These two peaks are separated by the $2 \mathrm{p}$ SOC $\zeta_{2 \mathrm{p}} \approx 13.5 \mathrm{eV}$, corresponding to the two resonant emission transitions from the intermediate state with $\mathscr{J}=1 / 2$ to the final states with $\mathscr{J}=1 / 2,3 / 2$.

4.1.1. Considering a Cubic (Octahedral) Crystal Field. When the absorbing ion is embedded in a solid state, one has to take 


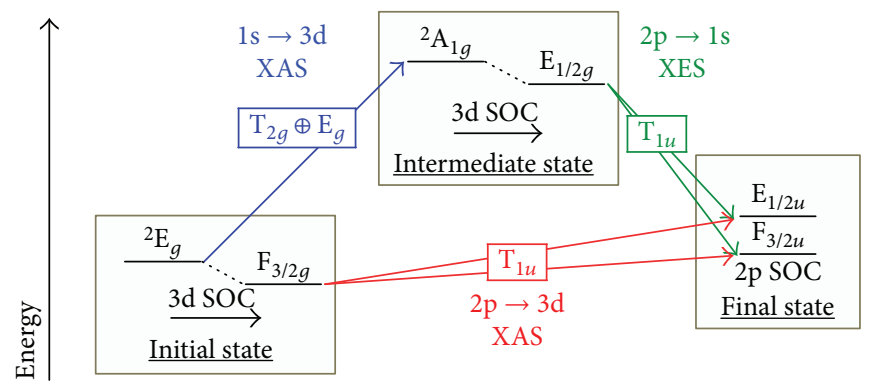

FIGURE 6: Multielectronic term scheme for $3 \mathrm{~d}^{9} \mathrm{Cu}^{2+}$ comparing the $1 \mathrm{~s} 2 \mathrm{p}$ RIXS and direct $2 \mathrm{p}$ XAS pathways. Where $\mathrm{SOC}(\mathcal{S}=1 / 2) \rightarrow \otimes \mathrm{E}_{1 / 2}$ is included, the spin multiplicity is omitted.

into account the crystal field potential created by the surrounding atoms. In the following, we will assume an octahedral $\left(\mathrm{O}_{h}\right)$ crystal field described by the parameter $10 \mathrm{D} q$. In a single electron picture, this crystal field induces the splitting of the $3 \mathrm{~d}$ orbitals into $t_{2 g}$ and $e_{g}$. In the multielectronic formalism (neglecting the $3 \mathrm{~d}$ spin-orbit coupling in first approximation), the ${ }^{2} \mathrm{D}$ term of the initial state branches in the $\mathrm{O}_{h}$ point group into the ${ }^{2} \mathrm{E}_{g}\left(\left|t_{2 g}^{6} e_{g}^{3}\right\rangle\right)$ and ${ }^{2} \mathrm{~T}_{2 g}\left(\left|t_{2 g}^{5} e_{g}^{4}\right\rangle\right)$ terms. Thus, the multielectronic ground state (at $T=0 \mathrm{~K}$ ) is ${ }^{2} \mathrm{E}_{g}\left(\mathrm{O}_{h}\right)$. The intermediate state term ${ }^{2} \mathrm{~S}\left(\mathrm{O}_{3}\right)$ branches in an octahedral crystal field into ${ }^{2} \mathrm{~A}_{1 g}\left(\mathrm{O}_{h}\right)$ symmetry.

In the $\mathrm{O}_{h}$ point group, the electric quadrupole 1s XAS operator has $\mathrm{T}_{2 g}$ and $\mathrm{E}_{g}$ symmetries. We derive the selection rules from the product table (Table 3 ) for the first step of the $1 \mathrm{~s} 2 \mathrm{p}$ RIXS process.

$$
\begin{aligned}
{ }^{2} \mathrm{E}_{g} \otimes \mathrm{T}_{2 g} & ={ }^{2} \mathrm{~T}_{1 g} \oplus^{2} \mathrm{~T}_{2 g}, \\
\underbrace{2}_{1 \mathrm{~s}^{2} 3 \mathrm{~d}^{9}} \mathrm{E}_{g} & \otimes \underbrace{\mathrm{E}_{g}}_{1 \mathrm{XXS}}=\underbrace{{ }^{2} \mathrm{~A}_{1 g} \oplus^{2} \mathrm{~A}_{2 g} \oplus{ }^{2} \mathrm{E}_{g}}_{\text {Accessible states }} .
\end{aligned}
$$

From (13), it becomes obvious that many symmetries are in principle accessible via 1s XAS from the multielectronic ground state symmetry ${ }^{2} \mathrm{E}_{g}\left(\mathrm{O}_{h}\right)$. In spite of the fact that many symmetries are in principle reachable via a quadrupole 1s XAS transition starting from the ground state symmetry ${ }^{2} \mathrm{E}_{g}\left(\mathrm{O}_{h}\right)$, the electronic structure of a $\mathrm{Cu}^{2+}$ ion only offers ${ }^{2} \mathrm{~A}_{1 g}\left(\mathrm{O}_{h}\right)$ symmetry leading to a single peak in the 1s XAS projection.

While spin-orbit coupling was neglected in this last step, it must be included in the description of the final state with the $2 p$ core hole to understand the complete $1 \mathrm{~s} 2 \mathrm{p}$ RIXS process and to be able to compare it with the 2 p XAS. Using the $\mathscr{L} . \mathcal{S}$-coupled terms obtained above in the atomic case, we derive their respective branchings in the octahedral crystal field $\left(\mathrm{O}_{h}\right)$.

The initial ground state ${ }^{2} \mathrm{D}_{5 / 2}\left(\mathrm{O}_{3}\right)$ branches in octahedral symmetry $\left(\mathrm{O}_{h}\right)$ into $\mathrm{E}_{5 / 2 g} \oplus \mathrm{F}_{3 / 2 g}\left(\mathrm{O}_{h}\right)$. The ground state at $T=0 \mathrm{~K}$ is ${ }^{2} \mathrm{E}_{g}\left[\mathrm{~F}_{3 / 2 g}\right]\left(\mathrm{O}_{h}\right)$. The effects of the $3 \mathrm{~d}$ spin-orbit coupling $\zeta_{3 \mathrm{~d}}$ and the $\mathrm{O}_{h}$ crystal field parameter $10 \mathrm{D} q$ are illustrated in Figure 2.

The intermediate state term ${ }^{2} \mathrm{~S}_{1 / 2}\left(\mathrm{O}_{3}\right)$ branches in an octahedral crystal field $\left(\mathrm{O}_{h}\right)$ into $\mathrm{E}_{1 / 2 g}\left(\mathrm{O}_{h}\right)$. The final states
${ }^{2} \mathrm{P}_{3 / 2}\left(\mathrm{O}_{3}\right)$ and ${ }^{2} \mathrm{P}_{1 / 2}\left(\mathrm{O}_{3}\right)$ branch into $\mathrm{F}_{3 / 2 u}\left(\mathrm{O}_{h}\right)$ and $\mathrm{E}_{1 / 2 u}$ $\left(\mathrm{O}_{h}\right)$, respectively. In octahedral symmetry, the electric dipole operator has $\mathrm{T}_{1 u}$ symmetry and thus, using the product table (Table 5), we find the reachable final states in the subsequent electric dipole emission ( $2 p$ XES):

$$
\mathrm{E}_{1 / 2 g} \otimes \mathrm{T}_{1 u}=\mathrm{E}_{1 / 2 u} \oplus \mathrm{F}_{3 / 2 u} .
$$

The direct $2 \mathrm{p}$ XAS starts from the $\mathrm{F}_{3 / 2 \mathrm{~g}}$ ground state yielding with the $\mathrm{T}_{1 u}$ IRREP the reachable final state terms:

$$
\mathrm{F}_{3 / 2 g} \otimes \mathrm{T}_{1 u}=\mathrm{E}_{1 / 2 u} \oplus \mathrm{E}_{5 / 2 u} \oplus 2 \mathrm{~F}_{3 / 2 u} .
$$

Altogether, this demonstrates that the crystal field enables both final states to be probed by $2 \mathrm{p}$ XAS and 1s2p RIXS. This is summarised in the scheme in Figure 6 and the corresponding calculated spectrum is shown in Figure 7.

The RIXS projections for various values of $10 \mathrm{D} q$ show almost no difference in both directions (XAS and XES). It is noteworthy that the $2 \mathrm{p}$ XAS $\left(\mathrm{L}_{2,3}\right.$-edge) approaches the two peaks in the $2 \mathrm{p}$ XES projections for large $10 \mathrm{D} q$ values. This is due to the crystal field $(10 \mathrm{D} q)$ affecting the spin-orbit interaction altering the $2 \mathrm{p}$ XAS selection rules.

In other words, the $\mathrm{L}_{2}$-edge probes in $2 \mathrm{p}$ XAS the amount of $\mathcal{J}=3 / 2$ character in the ground state. In the atomic case $(10 \mathrm{D} q=0.0 \mathrm{eV})$ with spherical symmetry $\left(\mathrm{O}_{3}\right)$ and at absolute zero $T=0 \mathrm{~K}$, the ion is pure $\mathscr{J}=5 / 2\left(\mathrm{D}_{5 / 2}\right)$. In octahedral $\mathrm{O}_{h}$ symmetry, on the other hand, an increasing crystal field (scaled via $10 \mathrm{D} q$ ) mixes more and more $\mathcal{J}=3 / 2$ character from the $\mathrm{D}_{3 / 2}$ into the ground state, resulting in a continuous visible increase of the $\mathrm{L}_{2}$ peak in 2p XAS.

And finally, it can be seen that the single-particle limit with two peaks is reached in both cases, 1s2p RIXS and $2 p$ XAS, respectively. The intensity ratio of the two peaks of $2: 1$ is given by the degeneracy of the ${ }^{2} \mathrm{P}_{1 / 2}\left(\mathrm{O}_{3}\right)$ and ${ }^{2} \mathrm{P}_{3 / 2}$ $\left(\mathrm{O}_{3}\right)$ final states.

4.1.2. $1 s 2 p$ RIXS Experiments of $3 d^{9}$ Systems. Experimental $1 s 2 p$ RIXS spectra of $\mathrm{CuO}$ have been published by Hayashi et al. [2]. They show the 1s2p X-ray emission spectra from excitation energies before the K-edge, through the edge to the continuum. As such, they observe the transition from resonances in the Lorentzian tails to nonresonant $1 \mathrm{~s} 2 \mathrm{p}$ XES. At the excitation energy at the pre-edge, the two-peaked $1 \mathrm{~s} 2 \mathrm{p}$ 


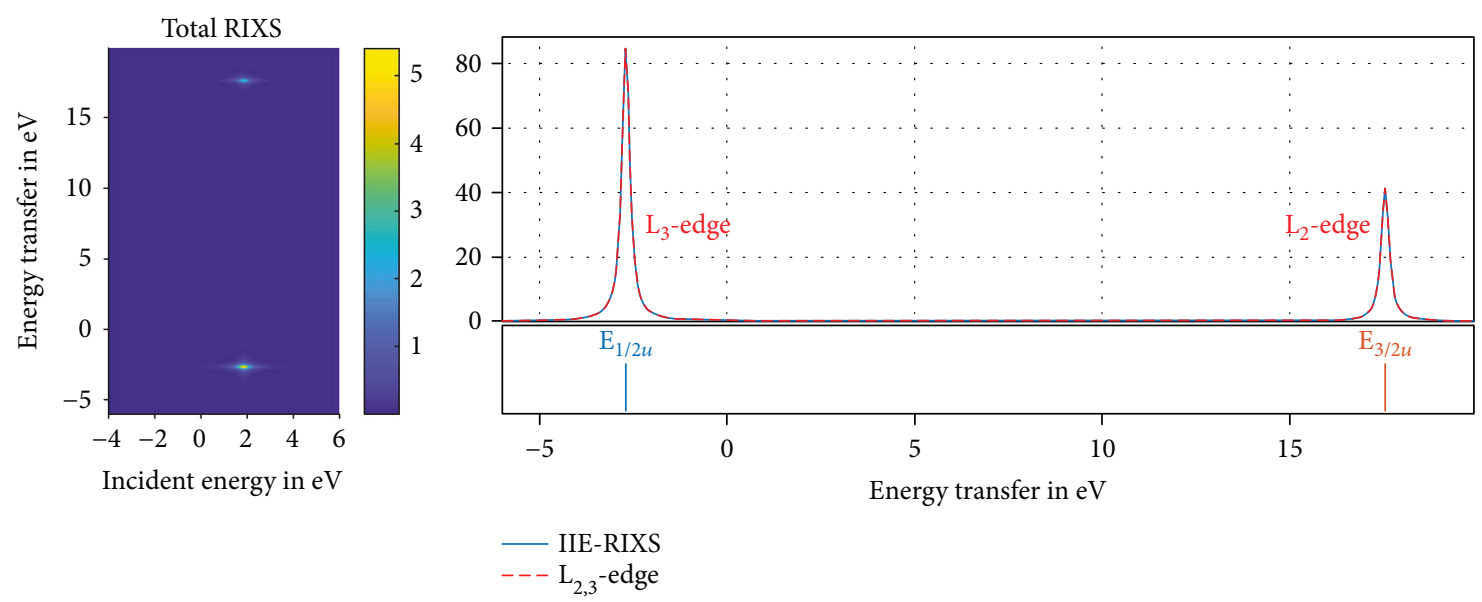

Figure 7: Calculated $1 \mathrm{~s} 2 \mathrm{p}$ RIXS map for $10 \mathrm{D} q=3.0 \mathrm{eV}$ with $2 \mathrm{p}$ XES projection for $\mathrm{Cu}^{2+}\left(3 \mathrm{~d}^{9}\right)$ for $10 \mathrm{D} q=3.0 \mathrm{eV}$. The $2 \mathrm{p}$ XES appears identical to the $2 \mathrm{p}$ XAS. The mixing induced by the crystal field symmetry breakdown enables to populate the $J=1 / 2$ and $J=3 / 2$ final states in direct $2 \mathrm{p}$ XAS.

RIXS spectrum is visible, in addition to the background from the edge [2].

4.2. $3 d^{0}$ : Tetravalent Titanium $\mathrm{Ti}^{4+}-1$ S XAS Peaks Split by $10 \mathrm{Dq}$. In the following, we discuss the differences between $2 \mathrm{p}$ XAS and $1 \mathrm{~s} 2 \mathrm{p}$ RIXS for the case of tetravalent titanium $\left(\mathrm{Ti}^{4+}\right)$. Here, the initial state configuration $1 \mathrm{~s}^{2} 2 \mathrm{p}^{6} 3 \mathrm{~d}^{0}$ has no partially filled shells. This is interesting because the resulting selection rules are straightforward: the nature of the probed final states reflects the nature and symmetry of the transition operators. The transition into the intermediate state promotes a 1 s electron into the $3 \mathrm{~d}$ band yielding a $1 \mathrm{~s}^{1} 2 \mathrm{p}^{6} 3 \mathrm{~d}^{1}$ electron configuration. The final state in $1 \mathrm{~s} 2 \mathrm{p}$ RIXS and $2 p$ XAS is in this case $1 s^{2} 2 p^{5} 3 d^{1}$.

$$
\begin{array}{r}
\text { 1s2p RIXS : } 1 \mathrm{~s}^{2} 2 \mathrm{p}^{6} 3 \mathrm{~d}^{0} \frac{\Delta \mathscr{J}= \pm 2}{1 \mathrm{sXAS}} 1 \mathrm{~s}^{1} 2 \mathrm{p}^{6} 3 \mathrm{~d}^{1} \frac{\Delta \mathscr{J}=0, \pm 1}{2 \mathrm{pXES}} 1 \mathrm{~s}^{2} 2 \mathrm{p}^{5} 3 \mathrm{~d}^{1}, \\
\text { 2p XAS : } 1 \mathrm{~s}^{2} 2 \mathrm{p}^{6} 3 \mathrm{~d}^{0} \stackrel{\Delta \mathscr{J}=0, \pm 1}{2 \mathrm{pXAS}} 1 \mathrm{~s}^{2} 2 \mathrm{p}^{5} 3 \mathrm{~d}^{1} .
\end{array}
$$

The $3 \mathrm{~d}^{0}$ case enables to describe the effect of the crystal field on the intermediate state, the interferences in the $1 \mathrm{~s} 2 \mathrm{p}$ RIXS process, and the more complex multielectronic effects in the final state. It provides an extent to $\mathrm{L}_{2,3}$-edge considerations of $3 \mathrm{~d}^{0}$ ions previously described in [35].

4.2.1. Atomic Case with Spin-Orbit Coupling. We start again with the case of an isolated $\mathrm{Ti}^{4+}$ ion $\left(3 \mathrm{~d}^{0}\right)$ where the effect of the solid state, that is, the crystal field, is neglected. The initial state configuration of $\mathrm{Ti}^{4+}$ is $1 \mathrm{~s}^{2} 2 \mathrm{p}^{6} 3 \mathrm{~d}^{0}$. Since all shells are full, $\mathcal{S}=0$ and $\mathscr{L}=0$. The $\mathscr{L} \cdot \mathcal{S}$-coupled total angular momentum is thus $\mathscr{J}=0$, and the initial state symmetry is the totally symmetric term ${ }^{1} \mathrm{~S}_{0}$.

The electronic configuration of the intermediate state in 1s 2 p RIXS of $\mathrm{Ti}^{4+}$ is $1 \mathrm{~s}^{1} 2 \mathrm{p}^{6} 3 \mathrm{~d}^{1}$, corresponding to the total orbital angular momentum $\mathscr{L}=2$ and total spin angular

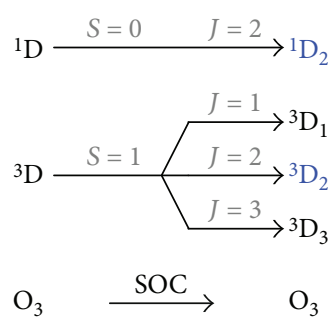

FIGURE 8: Splitting of the intermediate state spin singlet and triplet terms ${ }^{1,3} \mathrm{D}$ due to $3 \mathrm{~d}$ SOC $\zeta_{3 \mathrm{~d}}$ for a $\mathrm{Ti}^{4+}$ ion into $\mathrm{D}_{1}, \mathrm{D}_{2}$, and $\mathrm{D}_{3}$. States accessible in 1 s XAS $(\mathscr{J}=2)$ are shown in blue. However, the existing terms with $\mathscr{J}=1$ or 3 cannot be populated and will therefore not contribute to the spectrum.

momentum $\mathcal{S}=0$ or $\mathcal{S}=1$ giving the Russel-Saunders terms ${ }^{1} \mathrm{D}$ and ${ }^{3} \mathrm{D}(2 \cdot 10=20$ microstates $)$. Considering the spinorbit interaction in the $\mathscr{L} \cdot \mathcal{S}$ coupling scheme, one obtains $\mathscr{J}=2$ for the ${ }^{1} \mathrm{D}$ term and $\mathscr{J}=1,2$, and 3 for the ${ }^{3} \mathrm{D}$ term (Figure 8). The splitting of these four terms $\left({ }^{1} \mathrm{D}_{2},{ }^{3} \mathrm{D}_{1},{ }^{3} \mathrm{D}_{2}\right.$, and $\left.{ }^{3} \mathrm{D}_{3}\right)$ is defined by the $3 \mathrm{~d} \mathrm{SOC} \zeta_{3 \mathrm{~d}}$ and exchange interaction $\mathrm{G}_{\mathrm{sd}}^{2}$, which are both small ( $32 \mathrm{meV}$ and $46 \mathrm{meV}$, resp.). The calculated energy splitting is $\Delta E=92 \mathrm{meV}$ and is beyond the reach of current experimental resolution and not resolved in our calculations discussed here.

The electric quadrupole transition of the 1s XAS enables to reach the intermediate states with $\mathscr{J}=2$, though here $3 \mathrm{~d}$ SOC $\zeta_{3 \mathrm{~d}}$ is small and transitions to the spin triplet term ${ }^{3} \mathrm{D}_{2}$ will be weak, such that the 1s XAS is dominated by the transition in the spin singlet term ${ }^{1} \mathrm{D}_{2}$.

The final state electronic configuration is $1 s^{2} 2 p^{5} 3 d^{1}$ with two partially filled shells that have to be accounted for: the $2 \mathrm{p}$ and the $3 \mathrm{~d}$ shell. The total orbital angular moment $\mathscr{L}$ of the final state electronic configuration is $\mathscr{L}=1,2$, and 3 . The total spin angular moment $\mathcal{S}$ of the final state electronic configuration is $\mathcal{S}=0,1$. This gives in the atomic case the spin singlet and triplet terms ${ }^{1,3} \mathrm{P},{ }^{1,3} \mathrm{D}$, and ${ }^{1,3} \mathrm{~F}$. 


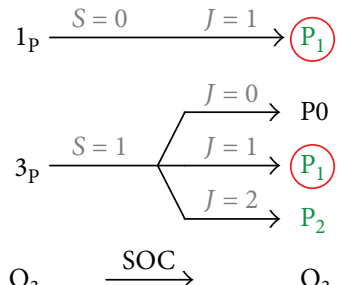

$\mathrm{O}_{3} \stackrel{\mathrm{SOC}}{\longrightarrow} \quad \mathrm{O}_{3}$

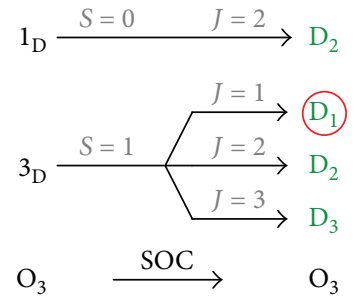

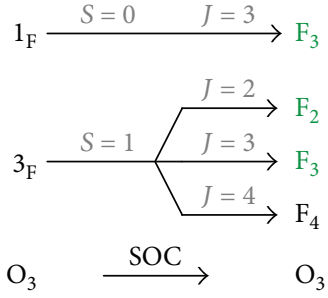

$\mathrm{O}_{3} \longrightarrow \mathrm{O}_{3}$

FIGURE 9: Splitting of the final state terms ${ }^{1,3} \mathrm{P},{ }^{1,3} \mathrm{D}$, and ${ }^{1,3} \mathrm{~F}$ due to spin-orbit coupling for $\mathrm{Ti}^{4+}$.
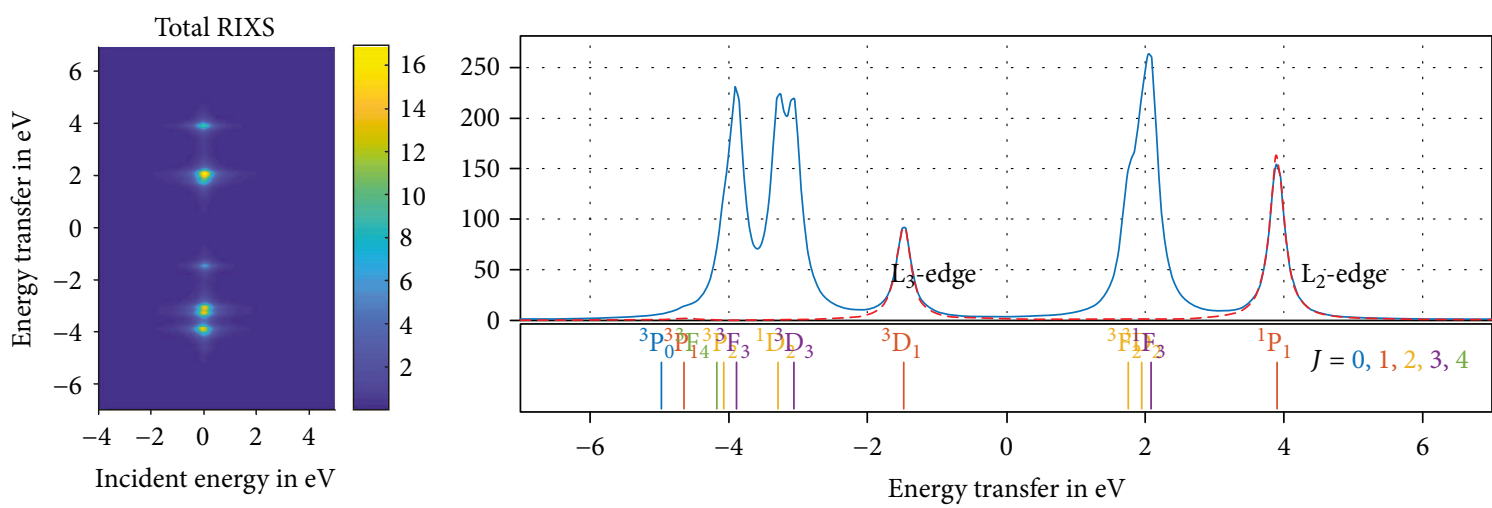

- IIE-RIXS
$---\mathrm{L}_{2,3}$-edge

Figure 10: Calculated 1s2p RIXS map with $2 \mathrm{p}$ XES projection and the $2 \mathrm{p}$ XAS $\left(\mathrm{L}_{2,3}\right.$-edge) for an isolated $\mathrm{Ti}^{4+}\left(3 \mathrm{~d}^{0}\right)$ ion in $\mathrm{O}_{3}$ symmetry.

As previously described by de Groot et al. [35], when spin-orbit interaction is neglected, the selection rules for the electric dipole transition in $2 \mathrm{p}$ XAS with $\Delta \mathcal{S}=0$ and $\Delta \mathscr{L}= \pm 1$ allow only to reach the singlet ${ }^{1} \mathrm{P}$ term from the ${ }^{1} \mathrm{~S}$ initial state, leading to one peak only (the $\mathrm{L}_{2}$-edge). The $\mathrm{L}_{3}$-edge, corresponding to transitions into the spin triplet term ${ }^{3} \mathrm{P}$, would have zero intensity. But in fact the strong 2 p spin-orbit coupling $\zeta_{2 p}$ in the final state ( $\mathscr{J}$ is used) leads to three peaks, two of them forming the $\mathrm{L}_{2}$ - and $\mathrm{L}_{3}$-edges, and a third weak peak related to triplet transitions.

The derivation of the total angular momentum $\mathscr{J}$ of the final state electronic configuration in the $\mathscr{L} . \mathcal{S}$ coupling scheme yields the term symbols illustrated in Figure 9.

The direct electric dipole transition in 2p XAS with $\Delta \mathscr{J}=0, \pm 1(\mathscr{J}=0 \rightarrow \mathscr{J}=0$ forbidden $)$ starts for $\mathrm{Ti}^{4+}$ from the total symmetric term ${ }^{1} \mathrm{~S}_{0}$, which allows to reach all final states with $\mathcal{J}=1\left(\mathrm{P}_{1}\right.$ and $\mathrm{D}_{1}$, marked with a red circle in Figure 9). The $\mathrm{L}_{2}{ }^{-}$and $\mathrm{L}_{3}$-edges are separated by the $2 \mathrm{p}$ spin-orbit coupling $\zeta_{2 \mathrm{p}}$ corresponding to transitions into the ${ }^{1} \mathrm{P}_{1}\left(\mathrm{~L}_{2}\right.$-edge $)$ and ${ }^{3} \mathrm{D}_{1}\left(\mathrm{~L}_{3}\right.$-edge $)$ terms. More precisely, from the squared matrix elements, we find that the second peak is dominated by ${ }^{3} \mathrm{D}_{1}$ contribution of $60 \%$, adding $36 \%$ of ${ }^{1} \mathrm{P}_{1}$ and $4 \%$ of ${ }^{3} \mathrm{P}_{1}$ character. The small third peak in the $2 \mathrm{p}$ XAS, at $E_{T} \approx-5 \mathrm{eV}$ in Figure 10, is related to transitions into the ${ }^{3} \mathrm{P}_{1}$ terms. Though the resolution chosen in our calculations does not reveal them as individual peaks, the direct Coulomb and exchange terms are not negligible. As discussed by de Groot et al. [35], they lead to a splitting of the $\mathrm{L}_{2,3^{-}}$ edge into three absorption lines including a redistribution of the intensities.

In the $1 \mathrm{~s} 2 \mathrm{p}$ RIXS process, the $2 \mathrm{p}$ XES decays with $\Delta \mathscr{J}=0, \pm 1$ starting from the intermediate states with $\mathscr{J}=$ $2\left(\mathrm{D}_{2}\right)$ and enables to reach the final states $\mathrm{P}_{1,2}, \mathrm{D}_{1,2,3}$, and $\mathrm{F}_{2,3}$ with $\mathcal{J}=1,2$, and 3 (terms shown in green Figure 9). Therefore, all $\mathscr{J}$ values except 0 and 4 are reachable.

With this, one can draw for $\mathrm{Ti}^{4+}$ the atomic term scheme with spin-orbit coupling for the two-step 1s2p RIXS and the one-step 2p XAS process as shown in Figure 11.

The intermediate state is the key element when comparing the direct $2 \mathrm{p}$ XAS and $1 \mathrm{~s} 2 \mathrm{p}$ RIXS. It enables to access additional terms in the $1 \mathrm{~s} 2 \mathrm{p}$ RIXS final state, adding multiple visible peaks in the energy transfer direction as shown in Figure 10. But in both cases, $\mathscr{J}=0$ and 4 are not possible to reach.

In summary, so far, we have discussed the effect of the spin-orbit coupling in the atomic case in spherical symmetry $\left(\mathrm{O}_{3}\right)$, but neglecting any crystal field. With the above, one can describe the 1s XAS with only one visible peak due to transitions into the ${ }^{1} \mathrm{D}_{2}$ intermediate state term. The $3 \mathrm{~d}$ spin-orbit coupling $\zeta_{3 \mathrm{~d}}$ and exchange interaction $\mathrm{G}_{\mathrm{sd}}$ lead to small splittings which are not visible in this plot. Furthermore, the small $3 \mathrm{~d}$ SOC may induce some mixing adding weak transitions into the ${ }^{3} \mathrm{D}_{2}$ term.

The splitting of the $2 \mathrm{p}$ XAS is dominated by the large $2 \mathrm{p}$ SOC $\zeta_{2 p}$ separating the $\mathrm{L}_{2}$-edge from the $\mathrm{L}_{3}$-edge, for example, the spin singlet and the triplet states. The $2 \mathrm{p}$ XES on the other hand consists of transitions from the intermediate 


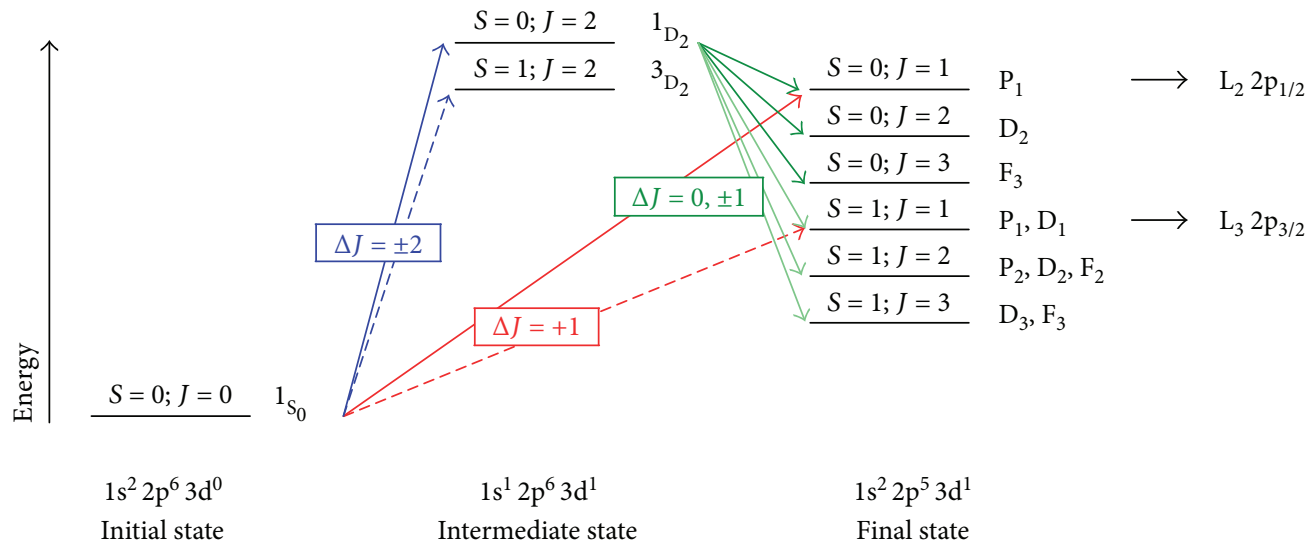

FIGURE 11: Atomic term scheme with spin-orbit coupling for $\mathrm{Ti}^{4+}\left(3 \mathrm{~d}^{0}\right)$ comparing the two-step (1s XAS, $2 p$ XES) 1 s2p RIXS and the one-step 2p XAS pathways. The two 1s XAS transitions are separated by the small 3d SOC, Coulomb, and exchange interactions; hence, the splitting is small. The direct dipole transitions in 2 p XAS can only populate the final states with $\mathscr{J}=1$, while the dipole decays in 2 p XES can reach final states with $\mathscr{J}=1,2$, and 3 . However, terms with $\mathscr{J}=0$ or 4 cannot be populated in either case and thus do not contribute to the spectrum. Nonaccessible terms are omitted for clarity.

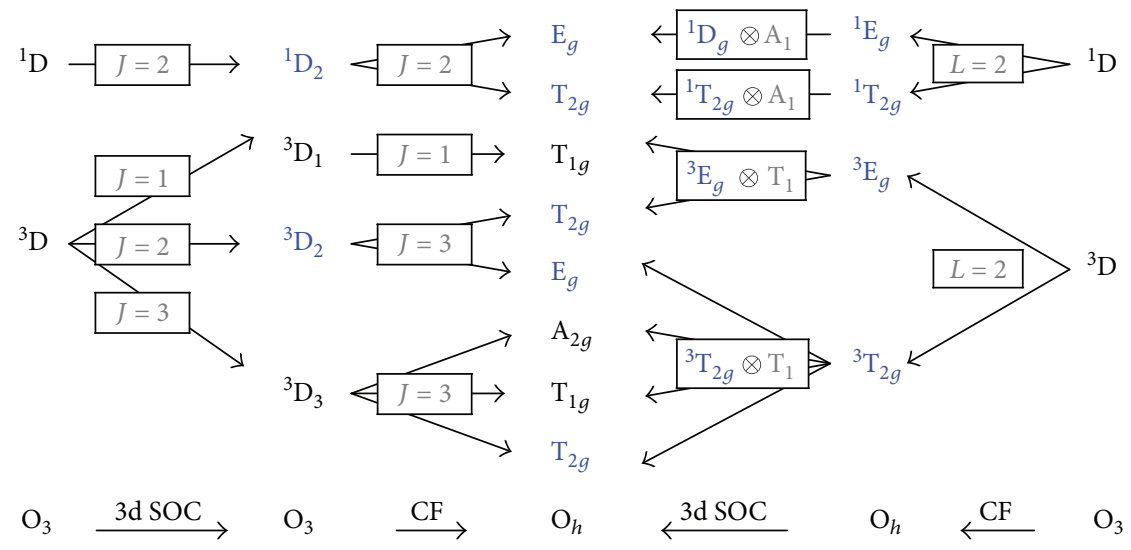

Figure 12: Branching of the intermediate state terms ${ }^{1} \mathrm{D}$ and ${ }^{3} \mathrm{D}$ of $\mathrm{Ti}^{4+}\left(3 \mathrm{~d}^{0}\right)$ due to $3 \mathrm{~d}$ SOC and an $\mathrm{O}_{h}$ CF. Symmetries accessible in the quadrupole 1s XAS step (GS $\rightarrow$ IS) are shown in blue.

state with $\mathscr{J}=2 \operatorname{IRREP}\left(\right.$ mostly ${ }^{1} \mathrm{D}_{2}$ ) into the final states with $\mathscr{J}=1,2$, and 3 IRREPs resulting multiple visible peaks in the energy transfer direction. All final state terms are also split by the exchange and direct Coulomb interactions, $G_{p d}$ and $\mathrm{F}_{\mathrm{pd}}$, respectively.

4.2.2. Considering an $\mathrm{O}_{h}$ Crystal Field. We now consider the effect of an octahedral $\mathrm{O}_{h}$ crystal field. The totally symmetric initial state term ${ }^{1} \mathrm{~S}_{0}$ branches in $\mathrm{O}_{h}$ into ${ }^{1} \mathrm{~A}_{1 g}\left[\mathrm{~A}_{1 g}\right]$. Formal consideration of an $\mathrm{O}_{h}$ crystal field and $3 \mathrm{~d}$ spin-orbit interaction leads to the branching of the intermediate state terms ${ }^{1} \mathrm{D}$ and ${ }^{3} \mathrm{D}$ into eight terms. The scheme in Figure 12 illustrates these branchings of the terms due to an $\mathrm{O}_{h}$ crystal field and the $3 \mathrm{~d}$ spin-orbit interaction $\zeta_{3 \mathrm{~d}}\left(\mathcal{S}=0 \rightarrow \otimes \mathrm{A}_{1 \mathrm{~g}}\right.$ and $\left.\mathcal{S}=1 \rightarrow \otimes \mathrm{T}_{1 g}\right)$.

In spite of several available intermediate state terms, starting from the initial state ${ }^{1} \mathrm{~A}_{1 g}$ and using the $\mathrm{T}_{2 g}$ and $\mathrm{E}_{g}$ IRREPs, representing the quadrupole 1s XAS operator, and the product table (Table 3) reflecting the orbital momentum selection rules, one finds the accessible intermediate state symmetries.

$$
\begin{aligned}
& { }^{1} \mathrm{~A}_{1 g} \otimes \mathrm{T}_{2 g}={ }^{1} \mathrm{~T}_{2 g}, \\
& \underbrace{{ }^{1} \mathrm{~A}_{1 g}}_{1 \mathrm{~s}^{2} 3 \mathrm{~d}^{0}} \otimes \underbrace{\mathrm{E}_{g}}_{\text {As XAS }}=\underbrace{{ }^{1} \mathrm{E}_{g}}_{\text {Accessible terms }} .
\end{aligned}
$$

In other words, from the totally symmetric ground state $\mathrm{A}_{1 g}\left(\mathrm{O}_{h}\right)$, only the $\mathrm{E}_{g}\left(\mathrm{O}_{h}\right)$ and $\mathrm{T}_{2 g}\left(\mathrm{O}_{h}\right)$ symmetries can be reached in the intermediate state via a quadrupole 1s XAS transition. Hence, for sufficiently large values of $10 \mathrm{D} q$, relative to the broadenings used in a calculation (or the experimental resolution in a measurement), two distinct peaks can be observed in the 1s XAS projection (Figure 13). Because the $3 \mathrm{~d}$ spin-orbit coupling $\zeta_{3 \mathrm{~d}}$ is small, the transitions to the spin singlet intermediate states are dominating (i.e., matrix elements are the largest), while the transitions via the $J=2$ IRREP from triplet ${ }^{3} \mathrm{D}$ should be weak. The $1 \mathrm{~s}$ 


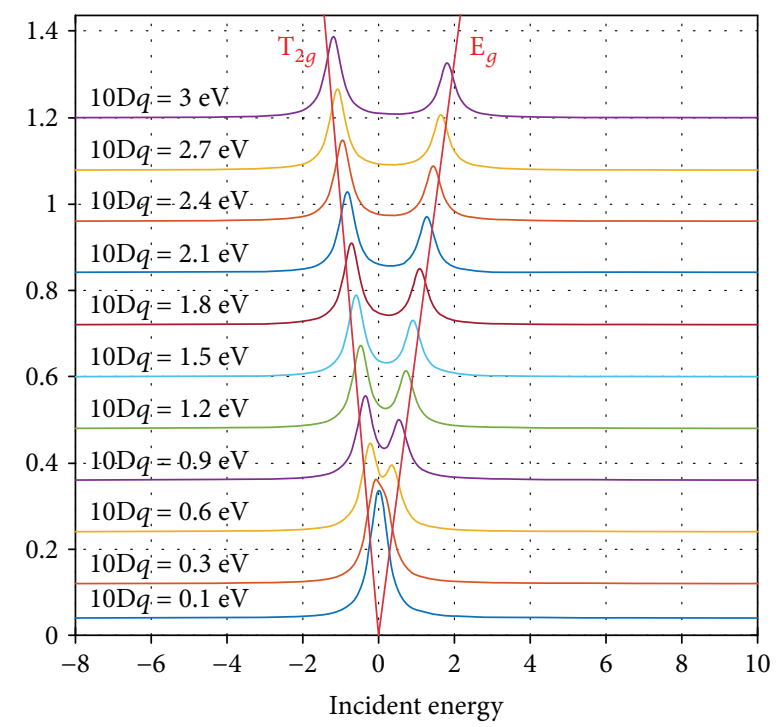

FIgURE 13: Calculated 1s XAS projections of a 1s2p RIXS for $\mathrm{Ti}^{4+}$ $\left(3 \mathrm{~d}^{0}\right)$ scaling the octahedral crystal field parameter $10 \mathrm{Dq}$ from $0.1 \mathrm{eV}$ to $3.0 \mathrm{eV}$ with an energy level diagram as overlay. The splitting of the $3 \mathrm{~d}$ level into the two peaks $\mathrm{T}_{2 \mathrm{~g}}$ and $\mathrm{E}_{g}$ due to the crystal field is visible.

XAS is dominated by transitions to the $\mathrm{E}_{g}$ and $\mathrm{T}_{2 g}$ terms from the ${ }^{1} \mathrm{D}_{2}$.

In an $\mathrm{O}_{h}$ crystal field, the final state IRREPs with $\mathscr{J}=$ $0,1,2,3$ and 4 (see Figure 11) branch, respectively, into the $\mathscr{L}$.S -coupled terms: $\mathrm{A}_{1 u}, \mathrm{~T}_{1 u}, \mathrm{E}_{u} \oplus \mathrm{T}_{2 u}, \mathrm{~A}_{2 u} \oplus \mathrm{T}_{1 u} \oplus \mathrm{T}_{2 u}$, and $\mathrm{A}_{1 u} \oplus \mathrm{E}_{u} \oplus \mathrm{T}_{1 u} \oplus \mathrm{T}_{2 u}$. The $2 \mathrm{p}$ XES in 1s2p RIXS from the two intermediate state terms $\mathrm{T}_{2 g}$ and $\mathrm{E}_{g}$ via the electric dipole operator, described by the $\mathrm{T}_{1 u}$ IRREP, leads to

$$
\begin{aligned}
& \mathrm{T}_{2 g} \otimes \mathrm{T}_{1 u}=\mathrm{A}_{2 u} \oplus \mathrm{E}_{u} \oplus \mathrm{T}_{1 u} \oplus \mathrm{T}_{2 u}, \\
& \underbrace{\mathrm{E}_{g}}_{1 s^{1} 3 \mathrm{~d}^{1}} \otimes \underbrace{\mathrm{T}_{1 u}}_{2 \mathrm{p} \text { XES }}=\underbrace{\mathrm{T}_{1 u} \oplus \mathrm{T}_{2 u}}_{\text {Accessible final states }} .
\end{aligned}
$$

It is noteworthy here that only the intermediate state with $\mathrm{T}_{2 g}$ symmetry can access in the $2 \mathrm{p}$ XES decay the $\mathrm{A}_{2 u}$ and $\mathrm{E}_{u}$ final state symmetries. The $\mathrm{T}_{1 u}$ and $\mathrm{T}_{2 u}$ final state terms are however accessible from both $\mathrm{T}_{2 g}$ and $\mathrm{E}_{g}$ intermediate state symmetries. Furthermore, we note that the intermediate state symmetry $A_{1 u}$ is not reachable as the final state symmetry and will therefore not contribute to the $2 p$ XES spectrum.

On the other hand, in 2p XAS, the possible final states must have $\mathrm{T}_{1 u}$ symmetry; otherwise, the transition matrix element equals zero. As can be seen from the branchings of $\mathscr{J}\left(\mathrm{O}_{3}\right)$ in and octahedral crystal field (Table 2), the $\mathrm{T}_{1 u}$ IRREP can be reached not only from the $\mathscr{F}=1$ IRREP but also from the $\mathscr{J}=3$ and $\mathscr{J}=4$ IRREPs.

This results in only seven possible $\mathrm{T}_{1 u}$ IRREPs in $\mathrm{O}_{h}$ symmetry, or in other words, only seven final states are reachable among the 60 available. The $2 p$ XAS spectrum consists, in principle, of seven lines, as can be seen in Figure 14, where the calculated spectra for $\mathrm{Ti}^{4+}\left(3 \mathrm{~d}^{0}\right)$ for a crystal-field splitting of $10 \mathrm{D} q=2.1 \mathrm{eV}$ are shown. While only spin singlet states would be reached in the absence of $2 p$ SOC, the consideration of the strong spin-orbit coupling in the final state induces a mixing of the spin singlet and triplet states. This weakens the spin selection rule $\Delta \mathcal{S}=0$ allowing also transitions into former spin triplet states (e.g., ${ }^{3} \mathrm{~T}_{2 u},{ }^{3} \mathrm{~T}_{1 u},{ }^{3} \mathrm{E}_{u}$, and ${ }^{3} \mathrm{~A}_{2 u}$ ).

This enables to draw the electronic term scheme in Figure 15 illustrating the different pathways in 1s2p RIXS and $2 \mathrm{p}$ XAS for $3 \mathrm{~d}^{0} \mathrm{Ti}^{4+}$ in $\mathrm{O}_{h}$ symmetry. The accessible $1 s 2 p$ RIXS final state terms are given in (18), while, as discussed above, the $2 \mathrm{p}$ XAS can only reach final states with $\mathrm{T}_{1 u}$ symmetry $\left(\mathrm{A}_{1 g} \otimes \mathrm{T}_{1 u}=\mathrm{T}_{1 u}\right)$.

Similarly to the $3 \mathrm{~d}^{9}$ case $\left(\mathrm{Cu}^{2+}\right)$ discussed in Section 4.1, comparing the final states, it becomes clear once again that the key elements are the intermediate states from which the $2 \mathrm{p}$ XES decays and thus enables to reach more final states when compared to the direct $2 \mathrm{p}$ XAS.

This overall results in the 1s2p RIXS maps shown in Figure 14. The total RIXS map is decomposed into the $\mathrm{E}_{g}$ and $\mathrm{T}_{2 g}$ quadrupole absorption components. The selective absorption towards each intermediate state is obtained. Experimentally, this dichroic behaviour has been observed for $\mathrm{Ti}^{4+}$ in cubic oxides such as the prototypal $\mathrm{SrTiO}_{3}$ [36]. The 1s2p RIXS spectra calculated for each of the two absorption peaks are shown (Figure 14(a)), and the 60 final states are labelled with their total angular IRREP (Figure 14(b)). This enables to see that both, the 1s2p RIXS and 2p XAS, probe the $\mathrm{T}_{1 u}$ final state symmetry. We further observe that within the four main peaks in 2p XAS, the first peaks of the $\mathrm{L}_{3}\left(\right.$ at $\left.E_{T} \approx-3.5 \mathrm{eV}\right)$ and of the $\mathrm{L}_{2}\left(\right.$ at $\left.E_{T} \approx 2 \mathrm{eV}\right)$ are stronger via the $\mathrm{T}_{2 g}$ absorption channel of the 1s2p RIXS than the $\mathrm{E}_{g}$ and vice-versa: the second peaks of the $\mathrm{L}_{3}\left(E_{T} \approx-1 \mathrm{eV}\right)$ and of the $\mathrm{L}_{2}\left(\right.$ at $\left.E_{T} \approx 4.5 \mathrm{eV}\right)$ are stronger via the $\mathrm{E}_{g}$ absorption channel of the $1 \mathrm{~s} 2 \mathrm{p}$ RIXS than via the $\mathrm{T}_{2 g}$. This can be understood in a single electron picture with the crystal field splitting of the final state: the lower states of the $\mathrm{L}_{3}$-edge contain the $3 \mathrm{~d}$ single electron in the $t_{2 g}$ orbitals while the higher energy states have the $3 \mathrm{~d}$ electron in the $e_{g}$ orbitals and similarly for the $\mathrm{L}_{2}$-edge [37].

4.2.3. $1 s 2 p$ RIXS Experiments of $3 d^{0}$ Systems. Experimental 1s XAS spectra (e.g., HERFD) of titanium oxides in the solid state often show more than two peaks in the pre-edge, which contradicts the result shown in Figure 13. The reason is that there are additional dipole transitions at energies overlapping with the quadrupole prepeaks. These dipole peaks are related to so-called nonlocal peaks, where the $4 \mathrm{p}$ states from the absorbing ion mix with the $3 \mathrm{~d}$ states of the neighbouring ions [3]. In addition, we note that for high valent ions such as $\mathrm{Ti}^{4+}$, charge transfer is important for the detailed description of the 2p XAS and 1s2p RIXS spectral shapes [37].

Experimental 1s2p RIXS experiments have been published on $\mathrm{TiO}_{2}$ by Glatzel et al. [38] and Kas et al. [39]. The $1 s 2 p$ RIXS planes show the quadrupole pre-edges and the nonlocal peaks. The 1s2p RIXS cross-section at the first quadrupole peak shows three peaks in qualitative agreement 

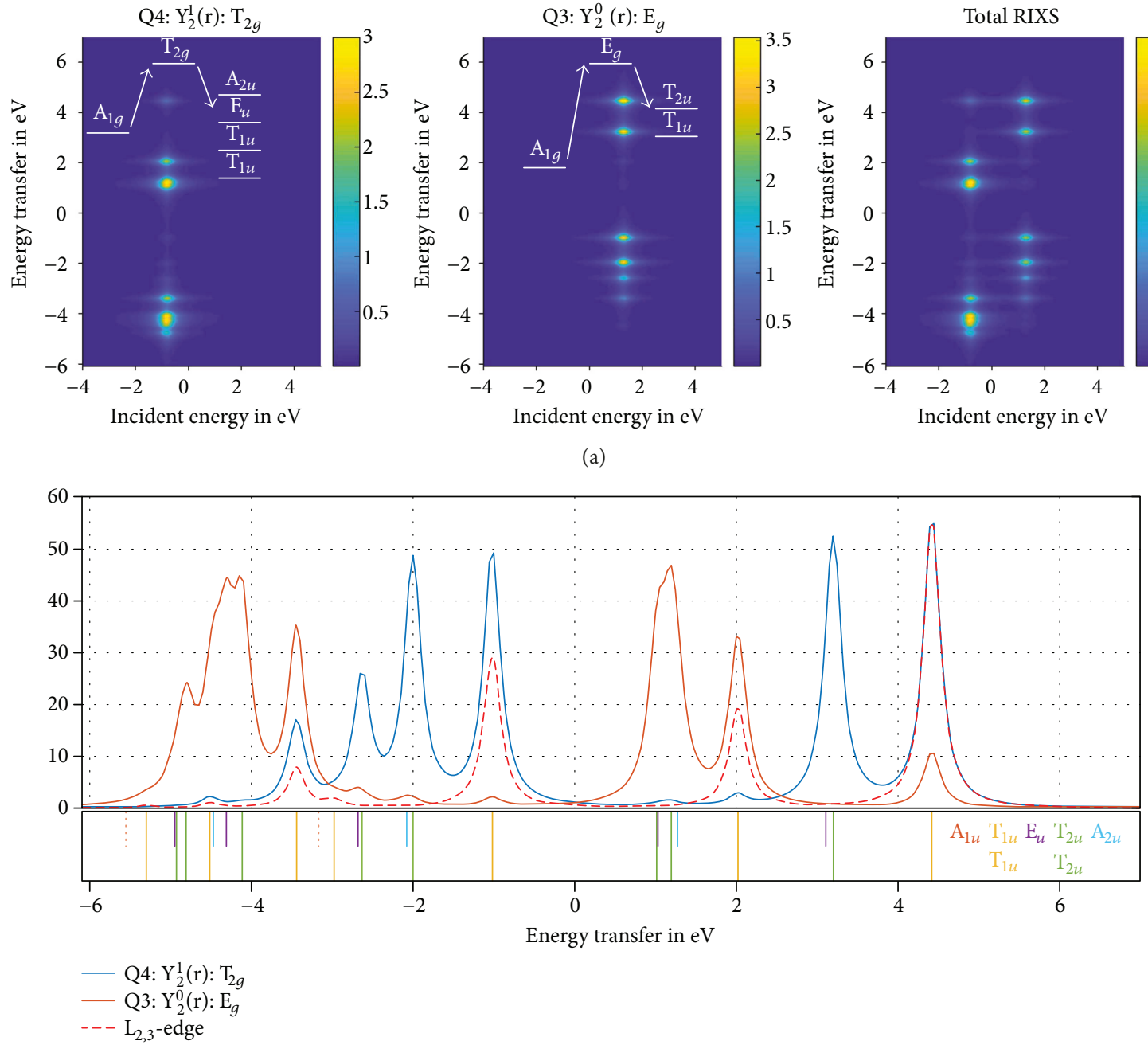

(b)

FIgURE 14: (a) Calculation of the $1 \mathrm{~s} 2 \mathrm{p}$ RIXS spectra for $\mathrm{Ti}^{4+}\left(3 \mathrm{~d}^{0}\right)$ with $10 \mathrm{D} q=2.1 \mathrm{eV}$ separated into the quadrupole components $Y_{l}^{m}$ (with $m=0,1$ and $l=2$ ) in spherical symmetry. They translate into the $\mathrm{T}_{2 g}$ and $\mathrm{E}_{g}$ IRREPs describing the quadrupole 1 s XAS transitions. The term scheme insets illustrate the corresponding pathways. (b) The corresponding $2 p$ XES projections onto the energy transfer axis for the two quadrupole components and the calculated 2 p XAS $\left(\mathrm{L}_{2,3}\right.$-edge).

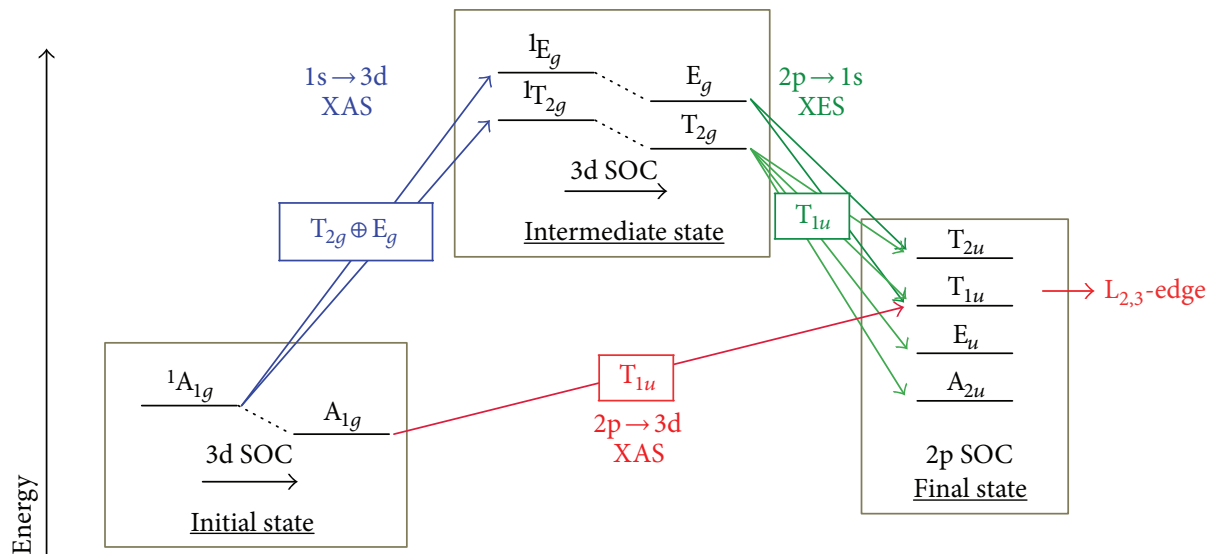

FIGURE 15: Multielectronic term scheme comparing the 2p XAS and the 1s2p RIXS pathways. The two quadrupole 1s XAS transitions with $\mathrm{T}_{2 g}$ and $\mathrm{E}_{g}$ symmetry lead to two different intermediate state symmetries. The subsequent dipole decays (2p XES) with $\mathrm{T}_{1 u}$ symmetry yield multiple 1s2p RIXS final state symmetries. The direct $2 \mathrm{p}$ XAS $\left(\mathrm{L}_{2,3}\right.$-edge) transition with $\mathrm{T}_{1 u}$ symmetry can on the other hand only access the $\mathrm{T}_{1 u}$ symmetry in the final state. Noncontributing states are omitted for clarity. 


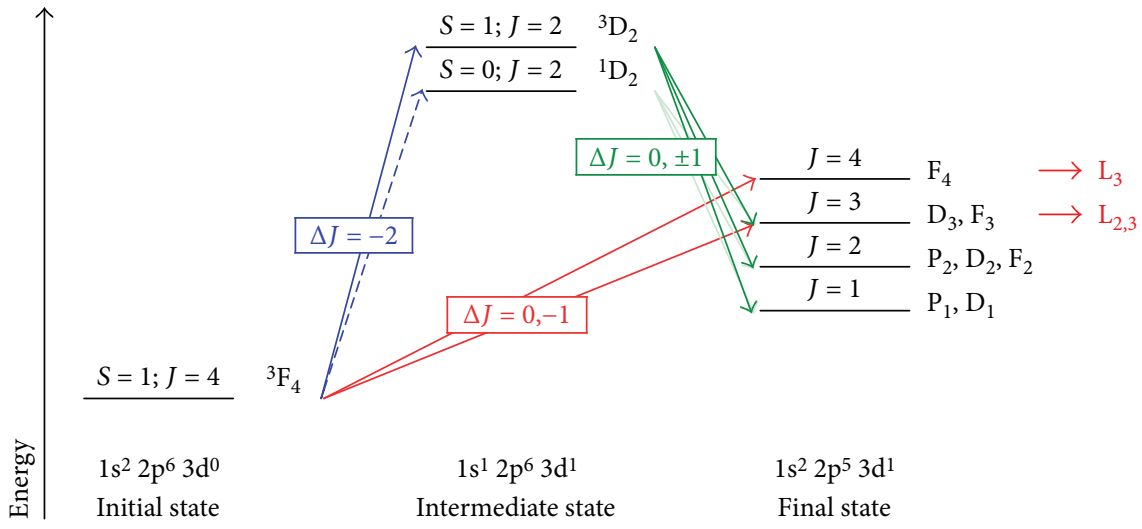

FIGURE 16: Atomic term scheme with spin-orbit coupling for $\mathrm{Ni}^{2+}$ comparing the two-step (1s XAS, $2 \mathrm{p}$ XES) $1 \mathrm{~s} 2 \mathrm{p}$ RIXS and the one-step $2 \mathrm{p}$ XAS pathways. Here, it should be noted that the two IS terms are only separated by the small $3 \mathrm{~d} S O C \zeta_{3 \mathrm{~d}}$. Note that the final state is similar to $\mathrm{Ti}^{4+}$. Nonaccessible terms (e.g., final states with $J=0$ ) are omitted for clarity.

with the $t_{2 g}$ calculation in Figure 14. Bagger et al. analysed the quadrupole and dipole RIXS separately [1]. We note that the ionic limit as calculated in Figure 14 is likely to be reached by 1 s2p RIXS experiments at the K-edge on divalent calcium systems $\left(\mathrm{Ca}^{2+}\right)$, including octahedral $\mathrm{CaO}$ and cubic $\mathrm{CaF}_{2}$.

4.3. $3 d^{8}$ : Divalent Nickel $\mathrm{Ni}^{2+}$-Single Peak in $1 \mathrm{~s}$ XAS. In this part, we investigate the elementary differences between $2 \mathrm{p}$ XAS and 1s2p RIXS for a divalent nickel ion $\left(\mathrm{Ni}^{2+}, 3 \mathrm{~d}^{8}\right)$. As we will show, the electronic configurations of the different steps of 1s2p RIXS present strong analogies with the case of $\mathrm{Ti}^{4+}$; the main difference being the nature of the initial state. We start again with the atomic case by deriving the $\mathscr{J}$ values as IRREPs.

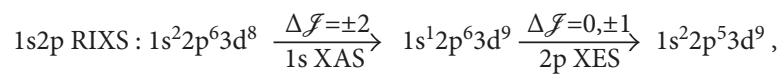

$$
\begin{aligned}
& 2 \mathrm{p} \mathrm{XAS}: 1 \mathrm{~s}^{2} 2 \mathrm{p}^{6} 3 \mathrm{~d}^{8} \longrightarrow \frac{\Delta \mathscr{J}=0, \pm 1}{2 \mathrm{pXAS}} \longrightarrow 1 \mathrm{~s}^{2} 2 \mathrm{p}^{5} 3 \mathrm{~d}^{9} .
\end{aligned}
$$

The electronic configuration of the initial state of $\mathrm{Ni}^{2+}$ is $1 \mathrm{~s}^{2} 2 \mathrm{p}^{6} 3 \mathrm{~d}^{8}$ with two holes in the $3 \mathrm{~d}$ shell. The multielectronic interactions lead to 45 states spread across the terms ${ }^{3} \mathrm{~F},{ }^{3} \mathrm{P},{ }^{1} \mathrm{G},{ }^{1} \mathrm{D}$, and ${ }^{1} \mathrm{~S}$ [13]. In spherical symmetry $\left(\mathrm{O}_{3}\right)$, the atomic ground state is ${ }^{3} \mathrm{~F}(\mathscr{L}=3$ and $\mathcal{S}=1)$. The $3 \mathrm{~d}$ spin-orbit coupling $\zeta_{3 \mathrm{~d}} \approx 0.1 \mathrm{eV}$ induces the splitting of the ${ }^{3} \mathrm{~F}$ term into the three terms:

$$
{ }^{3} \mathrm{~F} \stackrel{3 \mathrm{dSOC}}{\longrightarrow}{ }^{3} \mathrm{~F}_{2},{ }^{3} \mathrm{~F}_{3},{ }^{3} \mathrm{~F}_{4} \text {. }
$$

From those three IRREPs with $\mathcal{E}=2,3$, and 4 , the ${ }^{3} \mathrm{~F}_{4}$ term with $\mathscr{J}=4$ is the ground state term symbol.

The electronic configuration of the intermediate state in 1s2p RIXS of $\mathrm{Ni}^{2+}$ is $1 s^{1} 2 \mathrm{p}^{6} 3 \mathrm{~d}^{9}$ (one hole in the 1s shell and one hole in the $3 \mathrm{~d}$ shell) which translates into the two terms ${ }^{1} \mathrm{D}$ and ${ }^{3} \mathrm{D}(2 \cdot 10=20$ microstates $)$. It is noteworthy that this is identical to the intermediate state of $\mathrm{Ti}^{4+}$ described above (Section 4.2) due to the electron-hole equivalency. The $3 \mathrm{~d}$ spin-orbit interaction splits the two terms into the ${ }^{1} \mathrm{D}_{2}$ (for $\mathcal{S}=0$ ) and ${ }^{3} \mathrm{D}_{1},{ }^{3} \mathrm{D}_{2}$, and ${ }^{3} \mathrm{D}_{3}$ (for $\mathcal{S}=1$ ) Russel-Saunders terms. The resulting splittings distribute the four terms in energy over $\Delta E \approx 0.25 \mathrm{eV}$ for which the 1s XAS will appear only as a single peak due to the resolution chosen in our calculations, analogue to the $\mathrm{Ti}^{4+}$ atomic case.

The difference in the 1s XAS step in 1s2p RIXS between the $3 \mathrm{~d}^{8} \mathrm{Ni}^{2+}$ and $3 \mathrm{~d}^{0} \mathrm{Ti}^{4+}$ cases arises from the spin multiplicity of the initial state. In both cases, the electric quadrupole absorption leads to a $\mathscr{J}=2$ intermediate state. However, because of the small $3 \mathrm{~d}$ spin-orbit coupling $\left(\zeta_{3 \mathrm{~d}}=0.1 \mathrm{eV}\right)$, the spin multiplicity is mostly conserved in the transition and the absorption from the spin triplet initial state of $\mathrm{Ni}^{2+}$ leads to the ${ }^{3} \mathrm{D}_{2}$ state. For $\mathrm{Ti}^{4+}$ on the other hand, mainly, the ${ }^{1} \mathrm{D}_{2}$ term is reached.

The electronic configuration of the final state in 2p XAS and $1 s 2 p$ RIXS of $\mathrm{Ni}^{2+}$ is $1 \mathrm{~s}^{2} 2 \mathrm{p}^{5} 3 \mathrm{~d}^{9}$. This is again strictly analogue to the final state of $\mathrm{Ti}^{4+}$. The final state configuration corresponds to several atomic terms $\left({ }^{1,3} \mathrm{P},{ }^{1,3} \mathrm{D}\right.$, and $\left.{ }^{1,3} \mathrm{~F}\right)$ which are split further due to the strong $2 \mathrm{p}$ spin-orbit coupling $\zeta_{2 \mathrm{p}}=11.5 \mathrm{eV}$. The total symmetry final state terms are represented with integer $\mathcal{J}$ values ranging from $\mathcal{J}=0$ to $\mathscr{J}=4$. The detailed splittings are given above in Figure 9 in Section 4.2.

Only the final state terms with $\mathscr{J}=1,2$, and 3 are formally accessible in the 1s2p RIXS from the intermediate state with $\mathscr{J}=2$ via $2 \mathrm{p}$ XES decays $(\Delta \mathscr{F}=0, \pm 1)$. The direct $2 \mathrm{p}$ XAS (with $\Delta \mathscr{J}=0, \pm 1$ ) on the other hand can only reach the final state terms with $\mathscr{J}=3$ or $\mathscr{J}=4$ from the ground state with $\mathscr{J}=4$. Altogether, the selection rules for the atomic case can be summarised as

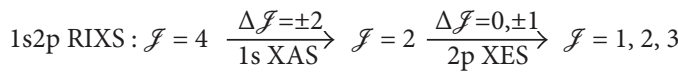

$$
\begin{aligned}
& \text { 2p XAS : } \mathscr{J}=4 \underset{\Delta \mathscr{J}=0, \pm 1}{2 \text { XAS }} \mathscr{J}=3,4 .
\end{aligned}
$$

This is also illustrated in the combined term scheme shown in Figure 16.

The calculated 1s2p RIXS of an isolated $\mathrm{Ni}^{2+}$ ion $\left(\mathrm{O}_{3}\right)$ is compared to the $2 \mathrm{p}$ XAS in Figure 17. The transitions are labelled with the dominant contribution of the 

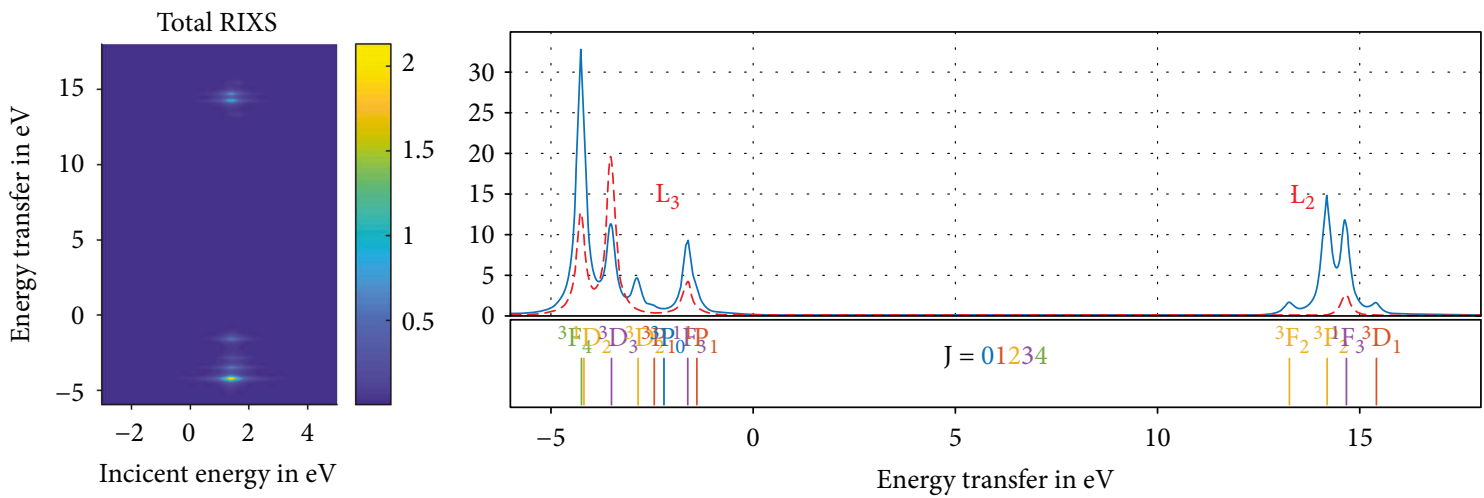

$\begin{array}{ll}- & \text { IIE-RIXS } \\ --- & L_{2,3} \text {-edge }\end{array}$

FIGURE 17: Calculated 1s2p RIXS for an isolated $\mathrm{Ni}^{2+}$ ion $\left(3 \mathrm{~d}^{8}\right)$ representing the atomic case. The projection of the total $1 \mathrm{~s} 2 \mathrm{p}$ RIXS map onto the energy transfer axis is shown together with the $2 \mathrm{p}$ XAS $\left(\mathrm{L}_{2,3}\right.$-edge) in dashed red and the corresponding stick spectrum.

corresponding final state terms. The four peaks in the $2 p$ XAS (three in the $\mathrm{L}_{3}$ and one in the $\mathrm{L}_{2}$ ) correspond to the $\mathscr{J}=3$ and $\mathcal{F}=4$ IRREPs. It is noteworthy here that the ${ }^{3} \mathrm{~F}_{4}$ final state term is special in the sense, that it can be accessed only in $2 \mathrm{p}$ XAS, but its population via $1 \mathrm{~s} 2 \mathrm{p}$ RIXS is forbidden. Hence, in $2 \mathrm{p}$ XAS, the peak at $E_{T} \approx-4 \mathrm{eV}$ is related to the $\mathscr{J}=4$ IRREP (dominated by ${ }^{3} \mathrm{~F}_{4}$ ), while in 1 s2p RIXS the peak at $E_{T} \approx-4 \mathrm{eV}$ in the $2 \mathrm{p}$ XES decay relates to transitions into the final state IRREP $\mathscr{J}=2\left(\operatorname{mostly}{ }^{1} \mathrm{D}_{2}\right)$. In other words, though it seems that the same peak at $E_{T} \approx-4 \mathrm{eV}$ appears in the $2 \mathrm{p}$ XAS as well as in the 2 p XES decay in 1 s 2 p RIXS, it is in each case related to a different final state IRREP.

Closer inspection of the sticks reveals that the ${ }^{3} \mathrm{~F}_{3}$ term does not appear as a dominant contribution, but instead, the ${ }^{1} \mathrm{~F}_{3}$ term appears twice. This is due to the fact that the atomic terms lose their meaning when spin-orbit interaction is included. Then $\mathscr{J}=3$ becomes the identifying IRREP which is formally a linear combination of ${ }^{1} \mathrm{~F}_{3},{ }^{3} \mathrm{D}_{3}$, and ${ }^{3} \mathrm{~F}_{3}$. Here, it happens to be the case that the ${ }^{1} \mathrm{~F}_{3}$ contribution is dominant in two $\mathscr{J}=3$ IRREPs.

As expected, the $\mathscr{F}=0$ IRREP (dominated by the ${ }^{3} \mathrm{P}_{0}$ term), as well as the $\mathscr{J}=1 \operatorname{IRREP}\left({ }^{3} \mathrm{P}_{1}\right.$ and ${ }^{3} \mathrm{D}_{1}$ terms), has no contribution to the $2 \mathrm{p}$ XAS spectrum. Furthermore, also, transitions into the $\mathscr{J}=2 \operatorname{IRREPs}\left(\mathrm{P}_{2}, \mathrm{~F}_{2}\right.$, and $\mathrm{F}_{2}$ terms $)$ are not appearing in $2 \mathrm{p}$ XAS.

In the 2 p XES decay, on the other hand, the $\mathscr{J}=2$ IRREPs have a much stronger contribution to the spectrum. Those peaks, related to the final state IRREPs $\mathscr{J}=2$, show in this example the largest difference between the 2p XAS and $2 \mathrm{p}$ XES as can be seen in Figure 17.

4.3.1. Considering an $\mathrm{O}_{h}$ Crystal Field. The crystal field splitting in $\mathrm{O}_{h}$ symmetry of the atomic terms of the initial state configuration of $\mathrm{Ni}^{2+}\left(1 \mathrm{~s}^{2} 2 \mathrm{p}^{6} 3 \mathrm{~d}^{8}\right)$ is well-known and described with the Tanabe-Sugano diagram. The ground state atomic term symbol ${ }^{3} \mathrm{~F}$ splits into ${ }^{3} \mathrm{~A}_{2 g},{ }^{3} \mathrm{~T}_{2 g}$, and ${ }^{3} \mathrm{~T}_{1 g}$ and gives ${ }^{3} \mathrm{~A}_{2 g}$ as the octahedral crystal field ground state. The inclusion of the $3 \mathrm{~d}$ spin-orbit coupling $\left(\mathcal{S}=1 \rightarrow \otimes \mathrm{T}_{1 g}\right)$ translates the ground state term as ${ }^{3} \mathrm{~A}_{2 g} \otimes \mathrm{T}_{1 g}=\mathrm{T}_{2 g}$. Thus,

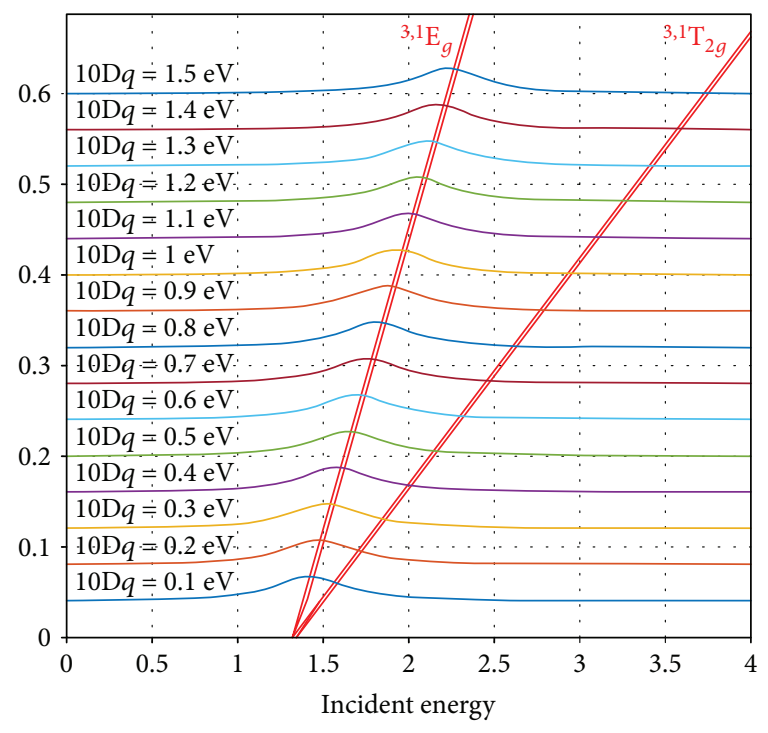

FIGURE 18: 1s XAS projections of the calculated 1s2p RIXS map for $\mathrm{Ni}^{2+}\left(3 \mathrm{~d}^{8}\right)$ showing only one peak as the $\mathrm{T}_{2 g}$ term is not reachable. The red lines indicate the energy levels of the intermediate state.

the crystal field ground state of $3 \mathrm{~d}^{8} \mathrm{Ni}^{2+}$ including $3 \mathrm{~d}$ spinorbit interaction is ${ }^{3} \mathrm{~A}_{2 g}\left[\mathrm{~T}_{2 g}\right]\left(\mathrm{O}_{h}\right)$.

The crystal field splitting of the 1s2p RIXS intermediate state of $\mathrm{Ni}^{2+}$ is analogue to $\mathrm{Ti}^{4+}$ as the ${ }^{1,3} \mathrm{D}$ terms split into ${ }^{1,3} \mathrm{E}_{g} \oplus{ }^{1,3} \mathrm{~T}_{2 g}$. From the ground state symmetry ${ }^{3} \mathrm{~A}_{2 g}$ (neglecting $3 \mathrm{~d}$ SOC), the $1 \mathrm{~s}$ XAS IRREPs $\mathrm{T}_{2 g}$ and $\mathrm{E}_{g}$ enable to reach only ${ }^{3} \mathrm{~T}_{1 g}$ and ${ }^{3} \mathrm{E}_{g}$ intermediate state symmetries:

$$
\begin{aligned}
& { }^{3} \mathrm{~A}_{2 g} \stackrel{\otimes \mathrm{T}_{2 g}}{\longrightarrow}{ }^{3} \mathrm{~T}_{1 g}, \\
& { }^{3} \mathrm{~A}_{2 g} \stackrel{\stackrel{\otimes \mathrm{E}_{g}}{\longrightarrow}}{\longrightarrow}{ }^{3} \mathrm{E}_{g} .
\end{aligned}
$$

In other words, the intermediate state term ${ }^{3} \mathrm{~T}_{2 g}$ cannot be reached by the electric quadrupole 1 s XAS absorption. 


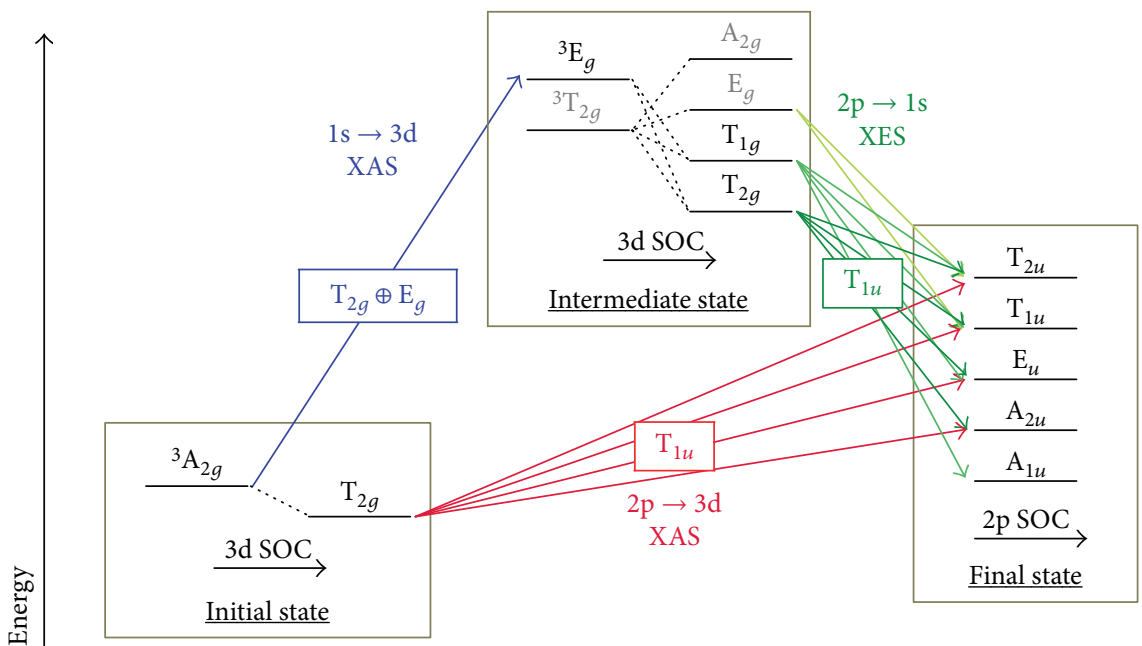

FIgure 19: Multielectronic term scheme for $\mathrm{Ni}^{2+}\left(3 \mathrm{~d}^{8}\right)$ comparing the $1 \mathrm{~s} 2 \mathrm{p}$ RIXS and direct $2 \mathrm{p}$ XAS pathways. Populating the $\mathrm{T}_{2 g}$ intermediate state would require a two-electron transition in $3 \mathrm{~d}^{8}$.

Hence, only the 1 s XAS IRREP $\mathrm{E}_{g}$ leads to an existing intermediate state symmetry: ${ }^{3} \mathrm{E}_{g}$. This is illustrated in Figure 18. As a result, the 1s XAS projection of the total 1s $2 p$ RIXS map will consist only of transitions into the ${ }^{3} \mathrm{E}_{g}$ intermediate state symmetry, because the quadrupole 1s XAS transition into the ${ }^{3} \mathrm{~T}_{2 g}$ intermediate state term is forbidden. We note that the spin singlet and triplet intermediate states are only separated by a few meV by the $3 \mathrm{~d}$ spin-orbit, Coulomb, and exchange interactions (e.g., $\zeta_{3 \mathrm{~d}} \approx 0.1 \mathrm{eV}$ ). Therefore, the $1 \mathrm{~s}$ XAS transitions appear as a single peak due to the resolution chosen in our calculations.

The single peak observed in the 1 s XAS step for $\mathrm{Ni}^{2+}$ $\left(3 d^{8}\right)$ is the first important difference with $\mathrm{Ti}^{4+}\left(3 \mathrm{~d}^{0}\right)$, which arises from the different nature of the initial state term. This can be further understood in a single electron picture: since the two holes of the initial state of $\mathrm{Ni}^{2+}$ are in the $e_{g}$ orbitals, the excited 1 s electron can only go to the empty $e_{g}$ orbitals $\left({ }^{3} \mathrm{E}_{g}\right.$ intermediate state $)$. On the contrary, in the case of $\mathrm{Ti}^{4+}$ (Section 4.2), both $e_{g}$ and $t_{2 g}$ orbitals are empty leaving both $\mathrm{E}_{g}$ and $\mathrm{T}_{2 g}$ intermediate state symmetries accessible.

When the crystal field $(10 \mathrm{D} q)$ is large with respect to the other electronic interactions, such as the $3 \mathrm{~d}$ spinorbit interaction $\zeta_{3 \mathrm{~d}}$, the 1s XAS step of the 1s $2 \mathrm{p}$ RIXS can be described well with the noncoupled IRREPs ( $\mathscr{L}$ and $\delta$ are good quantum numbers). However, when the crystal field is weak or when the electronic interactions are stronger, such as the $\zeta_{2 \mathrm{~d}}$ spin-orbit, it is necessary to use the $\mathscr{L} \cdot \mathcal{S}$-coupled terms. The spin-orbit coupling translates the intermediate state terms into the same total angular symmetry terms as for $\mathrm{Ti}^{4+}$ (see Figure 12). From this, it is evident that the intermediate state offers four different symmetries $\mathrm{A}_{2 g}, \mathrm{E}_{g}, \mathrm{~T}_{1 g}$, and $\mathrm{T}_{2 g}$ from which the $2 \mathrm{p}$ XES decays can occur.

The branching in the octahedral symmetry of the final state of $\mathrm{Ni}^{2+}$ is again analogue as $\mathrm{Ti}^{4+}$ (see Section 4.2). Those terms translate in an $\mathrm{O}_{h}$ crystal field to the ungerade symmetries: $\mathrm{A}_{1 u}, \mathrm{~A}_{2 u}, \mathrm{E}_{u}, \mathrm{~T}_{1 u}$, and $\mathrm{T}_{2 u}$. The dipole
$2 \mathrm{p}$ XES decays in $1 \mathrm{~s} 2 \mathrm{p}$ RIXS from the intermediate state terms give

$$
\begin{aligned}
& \mathrm{E}_{g} \stackrel{\otimes \mathrm{T}_{1 u}}{\longrightarrow} \mathrm{T}_{1 u} \oplus \mathrm{T}_{2 u} \\
& \mathrm{~T}_{1 g} \stackrel{\otimes \mathrm{T}_{1 u}}{\longrightarrow} \mathrm{A}_{1 u} \oplus \mathrm{E}_{u} \oplus \mathrm{T}_{1 u} \oplus \mathrm{T}_{2 u}, \\
& \mathrm{~T}_{2 g} \underset{2 \mathrm{p} \mathrm{XES}}{\stackrel{\otimes \mathrm{T}_{1 u}}{\longrightarrow}} \underbrace{\mathrm{A}_{2 u} \oplus \mathrm{E}_{u} \oplus \mathrm{T}_{1 u} \oplus \mathrm{T}_{2 u}}_{\text {Accessible FS terms }} .
\end{aligned}
$$

From that, we find that in $2 \mathrm{p}$ XES all final state symmetries can be reached. However, for $2 \mathrm{p}$ XAS, the dipole transition $\left(\mathrm{T}_{1 u}\right)$ starts from the $\mathrm{T}_{2 g}$ ground state.

$$
\mathrm{T}_{2 g} \underset{2 \mathrm{pXAS}}{\stackrel{\otimes \mathrm{T}_{1 u}}{\longrightarrow}} \underbrace{\mathrm{A}_{2 u} \oplus \mathrm{E}_{u} \oplus \mathrm{T}_{1 u} \oplus \mathrm{T}_{2 u}}_{\text {Accessible FS terms }} .
$$

From that, we find that the reachable $2 \mathrm{p}$ XAS final states can have $\mathrm{A}_{2 u}, \mathrm{E}_{u}, \mathrm{~T}_{1 u}$, or $\mathrm{T}_{2 u}$ symmetry. In other words, all intermediate terms, except those with $\mathrm{A}_{1 u}$, can be reached in $2 \mathrm{p}$ XAS. This is identical to the third case of the RIXS as described above in (26). However, the difference between the transition matrix elements of XAS and RIXS can result in different intensities.

The comparison between the 2p XAS and 1s $2 p$ RIXS selection rules is summarised in the scheme in Figure 19.

Comparing this case with the case of $\mathrm{Ti}^{4+}$ in an $\mathrm{O}_{h}$ crystal field enables to highlight the crucial influence of the ground state symmetry.

We conclude with the calculated 1s 2 p RIXS maps and a comparison with the corresponding $2 \mathrm{p}$ XAS as shown in Figure 20.

The calculations reveal two aspects: first, we notice the direct $2 \mathrm{p}$ XAS and the $2 \mathrm{p}$ XES final state spectra appear to have a similar appearance. This can be explained with the term scheme in Figure 19 illustrating that the 2p XES and the direct $2 \mathrm{p}$ XAS probe similar final state terms. Second, 

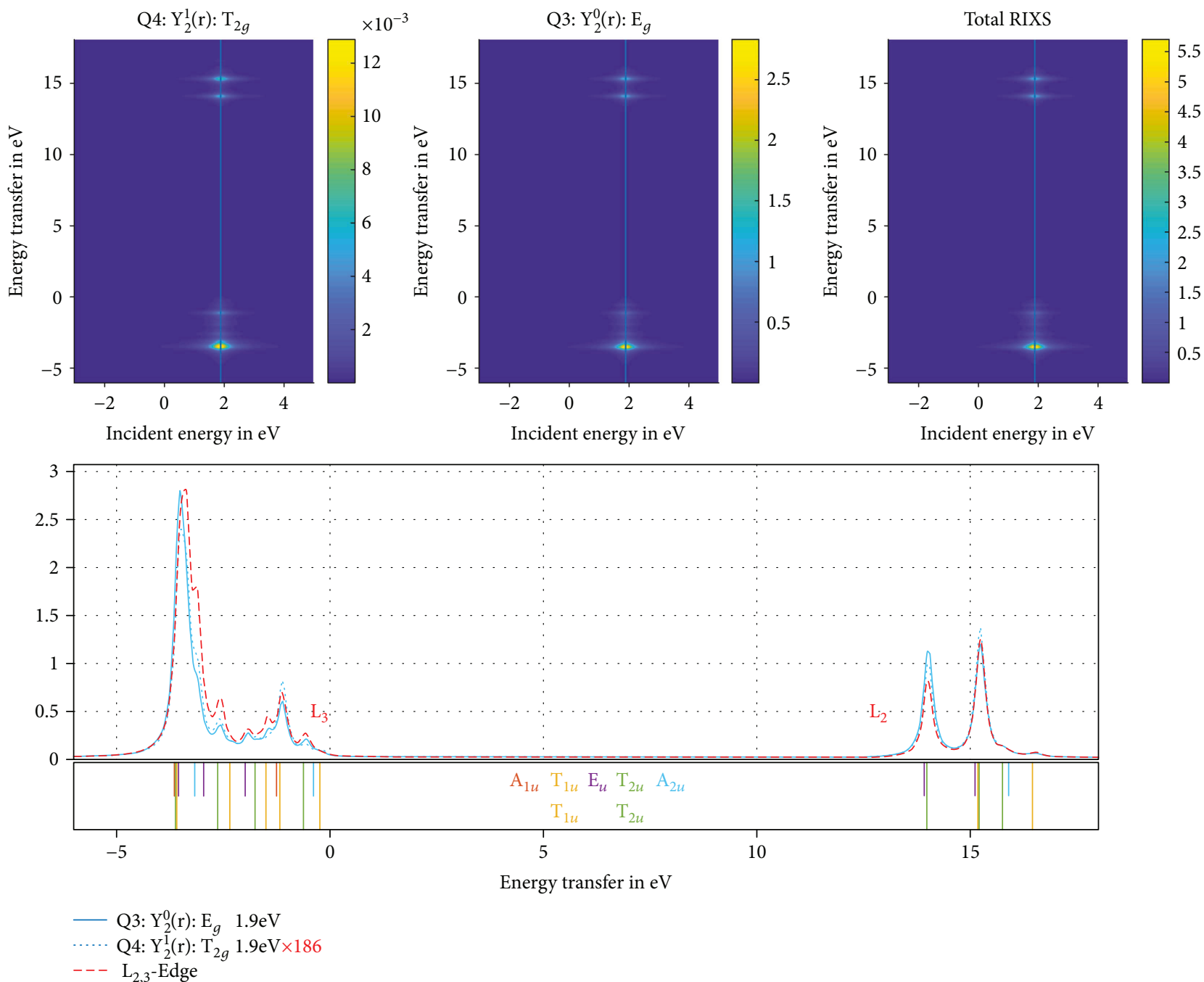

FIgURE 20: Calculated $1 \mathrm{~s} 2 \mathrm{p}$ RIXS for the ion $\mathrm{Ni}^{2+}\left(3 \mathrm{~d}^{8}\right)$ for $10 \mathrm{D} q=0.9 \mathrm{eV}$. As can be seen in the map reflecting the quadrupole IRREP T $2 g$ (top row, left), the contribution to the spectrum is nonzero because of the small $3 \mathrm{~d}$ spin-orbit interaction leading to a weak mixing of the states. However, its contribution is about 186 times weaker and thus neglectable in first approximation.

we note that the intensity of the contribution to the $1 \mathrm{~s} 2 \mathrm{p}$ RIXS via the quadrupole $\mathrm{T}_{2 g}$ IRREP is two orders of magnitude weaker than the contribution via $\mathrm{E}_{g} 1 \mathrm{~s}$ XAS IRREP. According to the strict $\mathscr{L}= \pm 2$ selection rule (e.g., when neglecting 3d spin-orbit coupling), no transitions arise from the $\mathrm{T}_{2 g}$ absorption. However, the $3 \mathrm{~d}$ spin-orbit interaction $\zeta_{3 \mathrm{~d}}$ is in fact nonzero, though small. Hence, when looking at the total angular momentum IRREPs of the intermediate states (e.g., the terms including SOC) and the 1s XAS selection rules shown in (22), it appears that some intermediate states can be probed by the $\mathrm{T}_{2 g}$ operator. In other words, the small but nonzero $3 \mathrm{~d}$ spin-orbit coupling $\zeta_{3 \mathrm{~d}}$ induces some mixing such that the contributions to the spectrum via the $1 \mathrm{~s}$ XAS IRREP $\mathrm{T}_{2 g}$ will be nonzero but weak. A direct comparison of the two 1s XAS transitions with $\mathrm{T}_{2 g}$ and $\mathrm{E}_{g}$ symmetries shows that the $\mathrm{T}_{2 g}$ spectrum multiplied by 186 is almost identical to the $\mathrm{E}_{g}$ spectrum (Figure 20).
4.3.2. $1 s 2 p$ RIXS Experiments of $3 d^{8}$ Systems. Experimental $1 s 2 \mathrm{p}$ RIXS spectra of $3 \mathrm{~d}^{8} \mathrm{NiF}_{2}$ and molecular $\mathrm{Ni}^{2+}$ complexes have been published by Glatzel et al. [14, 40]. Within the resolution of the measurement, the $\mathrm{NiF}_{2}$ spectrum is exactly reproduced by the crystal field calculation [14].

\section{1s2p RIXS for Other $3 d^{N}$ Configurations}

In this section, the remaining $3 \mathrm{~d}^{N}$ configurations $(N \in$ $\{1, \ldots, 7\})$ are discussed in a more condensed manner. Furthermore, for the systems with a $3 d^{4}, 3 d^{5}, 3 d^{6}$, and $3 d^{7}$ ground state, a high spin (HS) and a low spin (LS) case is presented. This is due to the fact that the crystal field splitting energy $(10 \mathrm{D} q)$ and the pairing energy in those cases are competing. In other words, for sufficiently large values of $10 \mathrm{D} q$, the ground state changes from high spin to low spin. Subsequently, this affects the possible transitions within those systems and hence the resulting spectra will have a different appearance [13]. 
For each ion in high spin, we are choosing a $10 \mathrm{D} q$ value matching roughly the empirically found " $0.5-0.6 \mathrm{eV}$ per valency" approximation for transition metal oxides. To illustrate the differences, we additionally select for $3 d^{4}$ to $3 d^{7}$ a sufficiently large value of $10 \mathrm{D} q$ to obtain the corresponding spectra for a low spin configuration. The used crystal field values $(10 \mathrm{D} q)$ are summarised in Table 7.

As we have seen already throughout the didactic cases in Section 4, the $2 p$ XES decays are always described with the dipole IRREP $\mathrm{T}_{1 u}$. As this will be used in the following sections in a rather repetitive manner, in (28), (29), (30), (31), (32), (33), (34), and (35), we are summarising all the reachable final state symmetries for any given intermediate state symmetry (see direct product Table 4 ).

$$
\begin{aligned}
& \mathrm{A}_{1 g} \otimes \mathrm{T}_{1 u}=\mathrm{T}_{1 u}, \\
& \mathrm{~A}_{2 g} \otimes \mathrm{T}_{1 u}=\mathrm{T}_{2 u}, \\
& \mathrm{E}_{g} \otimes \mathrm{T}_{1 u}=\mathrm{T}_{1 u} \oplus \mathrm{T}_{2 u}, \\
& \mathrm{~T}_{1 g} \otimes \mathrm{T}_{1 u}=\mathrm{A}_{1 u} \oplus \mathrm{E}_{u} \oplus \mathrm{T}_{1 u} \oplus \mathrm{T}_{2 u}, \\
& \mathrm{~T}_{2 g} \otimes \mathrm{T}_{1 u}=\mathrm{A}_{2 u} \oplus \mathrm{E}_{u} \oplus \mathrm{T}_{1 u} \oplus \mathrm{T}_{2 u}, \\
& \mathrm{E}_{1 / 2 g} \otimes \mathrm{T}_{1 u}=\mathrm{E}_{1 / 2 u} \oplus \mathrm{F}_{3 / 2 u}, \\
& \mathrm{E}_{5 / 2 g} \otimes \mathrm{T}_{1 u}=\mathrm{E}_{5 / 2 u} \oplus \mathrm{F}_{3 / 2 u}, \\
& \mathrm{~F}_{3 / 2 g} \otimes \mathrm{T}_{1 u}=\mathrm{E}_{1 / 2 u} \oplus 2 \mathrm{~F}_{3 / 2 u} \oplus \mathrm{E}_{5 / 2 u} .
\end{aligned}
$$

As can be seen above, the first five equations, (28), (29), (30), (31), and (32), relate to intermediate states with an even number of electrons in open shells (e.g., integer $\delta$ value). The bottom three equations (33), (34), and (35) on the other hand relate to intermediate state symmetries with an electron configuration having an odd number of electrons in open shells (e.g. half-integer $\delta$ value). In the following, we prefer to refer to these equations instead of repeating them every time in each of the following cases.

5.1. $3 d^{1}$ Ground State System, for Example, $\mathrm{Ti}^{3+}$. In the previous cases, the splitting of the ground state electronic terms was leading to a single term, for example, ${ }^{2} \mathrm{E}_{g}\left[\mathrm{~F}_{3 / 2 g}\right]\left(\mathrm{O}_{h}\right)$ for $\mathrm{Cu}^{2+}\left(3 \mathrm{~d}^{9}\right)$ or ${ }^{3} \mathrm{~A}_{2 g}\left[\mathrm{~T}_{2 g}\right]\left(\mathrm{O}_{h}\right)$ for $\mathrm{Ni}^{2+}\left(3 \mathrm{~d}^{8}\right)$. In the following, we discuss the $3 \mathrm{~d}^{1}$ ground state configuration representing for instance the case of $\mathrm{a} \mathrm{Ti}^{3+}$ ion.

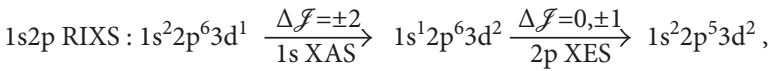

$$
\begin{aligned}
& 2 \mathrm{pXAS}: 1 \mathrm{~s}^{2} 2 \mathrm{p}^{6} 3 \mathrm{~d}^{1} \stackrel{\Delta \mathscr{f}=0, \pm 1}{2 \mathrm{pXAS}} \longrightarrow 1 \mathrm{~s}^{2} 2 \mathrm{p}^{5} 3 \mathrm{~d}^{2} .
\end{aligned}
$$

As shown below, the $3 \mathrm{~d}^{1}$ case is an interesting transition towards the other transition metal ions, as it enables to explore two aspects in the RIXS: (i) the influence of the spin-orbit splitting of the ground state leading to the contribution of several terms for $T>0 \mathrm{~K}$ and (ii) the effect of the multielectronic interactions of the $3 \mathrm{~d}$ shell in the intermediate and final states of $1 s 2 p$ RIXS. The atomic case will

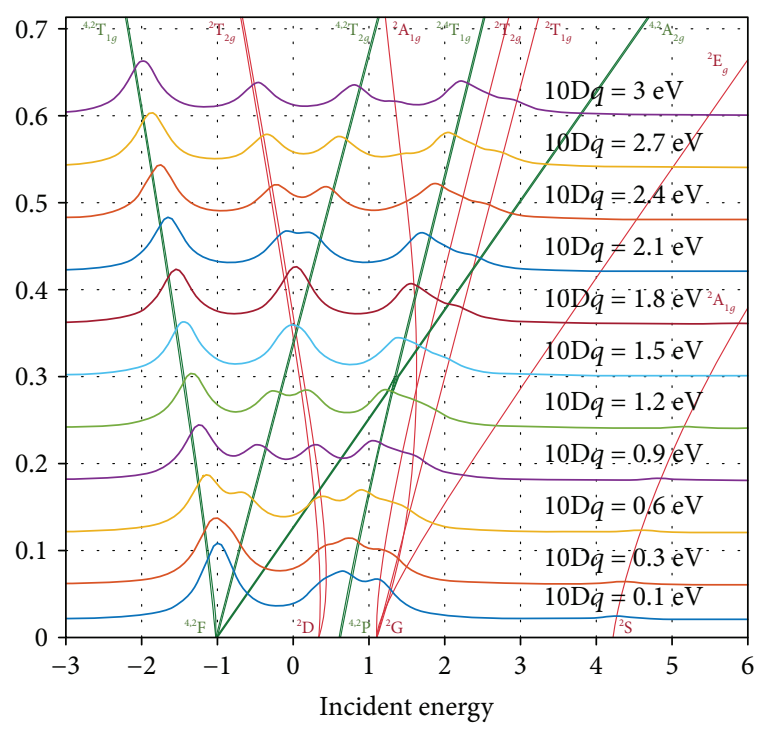

FIGURE 21: Influence of the $\mathrm{O}_{h}$ crystal field on the 1s XAS projection of the calculated $1 \mathrm{~s} 2 \mathrm{p}$ RIXS spectra for $\mathrm{Ti}^{3+}\left(3 \mathrm{~d}^{1}\right)$ with ELD overlay. A comparison to the calculated 1s XAS projections now shows on the one hand that neglecting $3 \mathrm{~d}$ SOC in this step is a valid approximation. And on the other hand, it confirms that a quadrupole transition from the ${ }^{2} \mathrm{~T}_{2 g}$ ground state symmetry can reach $\mathrm{A}_{1 g}, \mathrm{E}_{g}, \mathrm{~T}_{1 g}$, and $\mathrm{T}_{2 g}$ symmetries, but the $\mathrm{A}_{2 g}$ symmetry does apparently not contribute to the spectrum.

not be detailed, and we will only discuss the influence of the $\mathrm{O}_{h}$ crystal field.

The initial state electronic configuration $1 \mathrm{~s}^{2} 2 \mathrm{p}^{6} 3 \mathrm{~d}^{1}$ only contains a single electron in the $3 \mathrm{~d}$ shell. It is noteworthy that this electronic configuration is analogue to the initial state of $\mathrm{Cu}^{2+}$ : the atomic ground state term is ${ }^{2} \mathrm{D}$, which splits into $\mathrm{D}_{5 / 2}$ and $\mathrm{D}_{3 / 2}$ by the $3 \mathrm{~d}$ spin-orbit coupling (see Table 1 ). In the $\mathrm{O}_{h}$ crystal field, the ${ }^{2} \mathrm{D}$ term splits into ${ }^{2} \mathrm{~T}_{2 g}\left(\left|t_{2 g}^{1} e_{g}^{0}\right\rangle\right)$ and ${ }^{2} \mathrm{E}_{g}\left(\left|t_{2 g}^{0} e_{g}^{1}\right\rangle\right)$.

The difference with $\mathrm{Cu}^{2+}\left(3 \mathrm{~d}^{9}\right)$ is that the ground state term of $\mathrm{Ti}^{3+}\left(3 \mathrm{~d}^{1}\right)$ is ${ }^{2} \mathrm{~T}_{2 g}$ (6 microstates). When considering the $3 \mathrm{~d}$ spin-orbit coupling $\zeta_{3 \mathrm{~d}}$, this term splits into the two total symmetry irreducible representations (IRREPs) $\mathrm{F}_{3 / 2 \mathrm{~g}}$ (mixing of $\mathrm{D}_{5 / 2}$ and $\mathrm{D}_{3 / 2}$ ) and $\mathrm{E}_{5 / 2 \mathrm{~g}}$ (from $\mathrm{D}_{5 / 2}$ term), where $\mathrm{F}_{3 / 2 g}$ is the lowest. This is summarised in Figure 3, which shows the splittings of the ground state Russel-Saunders term ${ }^{2} \mathrm{D}$ with SOC and an $\mathrm{O}_{h}$ crystal field. It should be compared with $\mathrm{Cu}^{2+}$ (Figure 2). Furthermore, we note that due to the small $3 \mathrm{~d}$ SOC in the initial state $\left(\zeta_{3 \mathrm{~d}}=19 \mathrm{meV}\right)$, the splitting of the ground state ${ }^{2} \mathrm{~T}_{2 g}$ is only $28 \mathrm{meV}$. Thus, for temperatures $T>0 \mathrm{~K}$, the second IRREP should also be included according to the Boltzmann population. For instance, at $T=300 \mathrm{~K}$, Boltzmann population of the $\mathrm{F}_{3 / 2 g}$ (4-fold degenerate) is $86 \%$ and $14 \%$ for the $\mathrm{E}_{5 / 2 g}$ (2-fold degenerate). The contribution of the second term $\mathrm{E}_{5 / 2 \mathrm{~g}}$ is thus small but not entirely negligible.

The intermediate state electron configuration $1 \mathrm{~s}^{1} 3 \mathrm{~d}^{2}$ $(2 \cdot 45=90$ microstates $)$ is equivalent to the initial state of $\mathrm{Ni}^{2+}$ coupled to the $1 \mathrm{~s}$ core hole [13]. The atomic terms are 


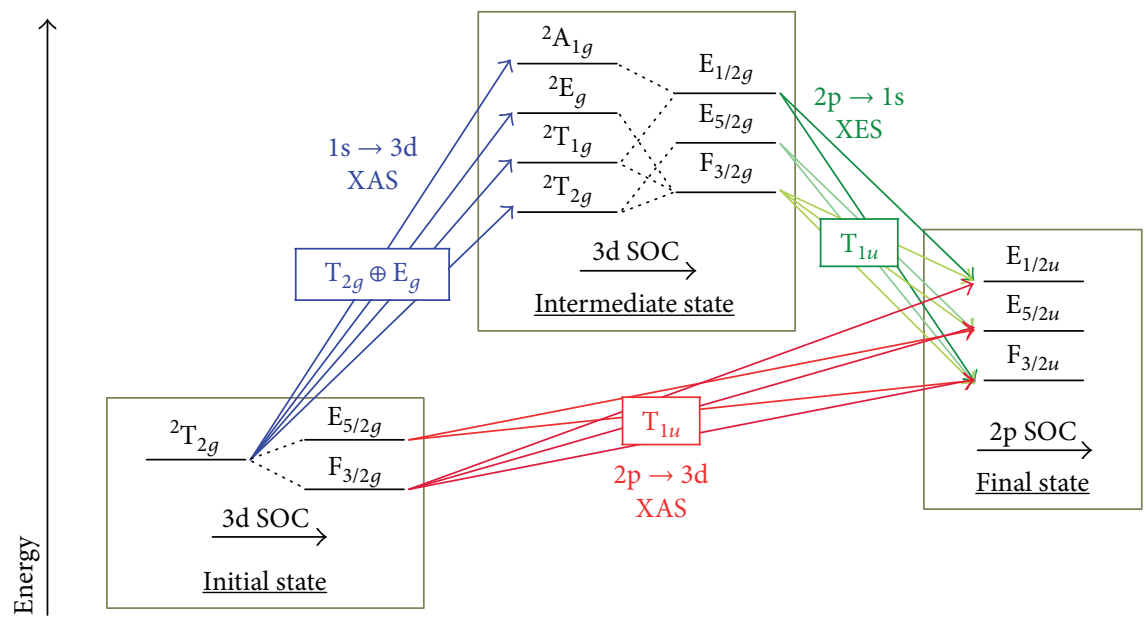

FIgURE 22: Multielectronic term scheme for $\mathrm{Ti}^{3+}\left(3 \mathrm{~d}^{1}\right)$ comparing the $1 \mathrm{~s} 2 \mathrm{p}$ RIXS and direct $2 \mathrm{p}$ XAS pathways. Where SOC is included, the spin multiplicity is omitted as its meaning is lost in that case.

${ }^{2,4} \mathrm{~F},{ }^{2,4} \mathrm{P},{ }^{2} \mathrm{G}$, and ${ }^{2} \mathrm{~S}$ (Table 1 ). Because the Coulomb exchange $G_{\text {sd }}$ is small, the splitting between the doublet and the quartet spin states is small and beyond the accessible energy resolution.

Using Table 2, one finds the branchings of the atomic states in an $\mathrm{O}_{h}$ crystal field. The resulting $\mathrm{O}_{h}$ term symbols for a $1 s^{1} 3 \mathrm{~d}^{2}$ configuration are ${ }^{2,4} \mathrm{~A}_{2 g},{ }^{2,4} \mathrm{~T}_{2 g},{ }^{2,4} \mathrm{~T}_{1 g},{ }^{2} \mathrm{~A}_{1 g}$, and ${ }^{2} \mathrm{E}_{g}$. The energy splitting of these $\mathrm{O}_{h}$ crystal field terms is qualitatively similar to the Tanabe-Sugano diagram of 3 $\mathrm{d}^{2}$. In the particular case of $\mathrm{Ti}^{3+}$, the result of the calculation is given in Figure 21 where the 1s XAS projections are shown together with the crystal field terms assigned.

Because the $3 \mathrm{~d}$ spin-orbit coupling is weak, one can neglect it in first approximation; the spin conservation leads to the 1s XAS being dominated by transitions into the spin doublet states, while the matrix elements of the transition to the spin quartet terms are much smaller. In the following, we will therefore focus on the doublet spin states.

$$
\begin{aligned}
& { }^{2} \mathrm{~T}_{2 g} \stackrel{\otimes \mathrm{T}_{2 g}}{\longrightarrow}{ }^{2} \mathrm{~A}_{1 g} \oplus^{2} \mathrm{E}_{g} \oplus{ }^{2} \mathrm{~T}_{1 g} \oplus^{2} \mathrm{~T}_{2 g} \\
& \underbrace{{ }^{2} \mathrm{~T}_{2 g}}_{\text {GS }} \underset{1 \mathrm{XXS}}{\stackrel{\otimes \mathrm{E}_{g}}{\longrightarrow}} \underbrace{{ }^{2} \mathrm{~T}_{1 g} \oplus^{2} \mathrm{~T}_{2 g}}_{\text {Reachable IS terms }} .
\end{aligned}
$$

This shows, in an $\mathrm{O}_{h}$ crystal field, starting from the ${ }^{2} \mathrm{~T}_{2 g}$ ground state, the $1 \mathrm{~s}$ XAS operators $\mathrm{T}_{2 g}$ and $\mathrm{E}_{g}$ enable to reach all even terms except the ${ }^{2} \mathrm{~A}_{2 g}$ IRREP. This results in numerous multiplet states reached in the intermediate state of $1 s 2 p$ RIXS. The 1s XAS projections in Figure 21 show the splitting of the peaks due to the scaling of the crystal field parameter $10 \mathrm{Dq}$. We note that the ${ }^{2} \mathrm{~A}_{2 g}\left({ }^{2} \mathrm{~F}\right)$ does not get any intensity in the absorption step of $1 \mathrm{~s} 2 \mathrm{p}$ RIXS in agreement with the 1s XAS selection rules as found in the expressions given in (37) and (38).

The previous didactic examples (Section 4) demonstrated the necessity to consider the $\mathscr{L} \cdot \mathcal{S}$-coupled terms to describe the $2 \mathrm{p}$ XES transitions to the final state in
$1 \mathrm{~s} 2 \mathrm{p}$ RIXS and the $2 \mathrm{p}$ XAS. In the intermediate state, the $\mathrm{O}_{h}$ total symmetry IRREPs of the doublet spin states are obtained by coupling the reachable orbital IRREPs with the spin IRREP $\left(\mathcal{S}=1 / 2 \rightarrow \otimes \mathrm{E}_{1 / 2 g}\right)$ :

$$
\begin{aligned}
& \mathrm{A}_{1 g} \otimes \mathrm{E}_{1 / 2 g}=\mathrm{E}_{1 / 2 g}, \\
& \mathrm{E}_{g} \otimes \mathrm{E}_{1 / 2 g}=\mathrm{F}_{3 / 2 g}, \\
& \mathrm{~T}_{1 g} \otimes \mathrm{E}_{1 / 2 g}=\mathrm{E}_{1 / 2 g} \oplus \mathrm{F}_{3 / 2 g}, \\
& \mathrm{~T}_{2 g} \otimes \mathrm{E}_{1 / 2 g}=\mathrm{E}_{5 / 2 g} \oplus \mathrm{F}_{3 / 2 g} .
\end{aligned}
$$

The result shows that all three odd total symmetric IRREPS $\mathrm{E}_{1 / 2 g}, \mathrm{~F}_{3 / 2 g}$, and $\mathrm{E}_{5 / 2 g}$ are thus reachable in the intermediate state. In other words, all three odd IRREPs are contributing to the $2 \mathrm{p}$ XES decays.

The final state configuration $1 \mathrm{~s}^{2} 2 \mathrm{p}^{5} 3 \mathrm{~d}^{2}$ contains $6 \cdot 45=270$ microstates due to the hole in the $2 \mathrm{p}$ shell. The atomic term symbols span from $\mathrm{S}$ to $\mathrm{H}$ with spin doublets and quartets and $\mathscr{J}$ values ranging from $1 / 2$ to $11 / 2$ [13]. In an $\mathrm{O}_{h}$ crystal field, these terms branch into the three IRREPs $\mathrm{E}_{1 / 2 u}, \mathrm{~F}_{3 / 2 u}$, and $\mathrm{E}_{5 / 2 u}$ (Table 2).

The $2 \mathrm{p}$ XES dipole transition IRREP $\mathrm{T}_{1 u}$ gives the transitions from the three intermediate state symmetries into all three final state symmetries as given in (33), (34), and (35).

For the direct $2 \mathrm{p}$ XAS, as mentioned above, the two ground state terms $\mathrm{F}_{3 / 2 g}$ and $\mathrm{E}_{5 / 2 g}$ are to be considered due to the small splitting. This leads with the dipole transition IRREP $\mathrm{T}_{1 u}$ also to all three final state symmetries:

$$
\begin{aligned}
& \mathrm{E}_{5 / 2 g} \stackrel{\otimes \mathrm{T}_{1 u}}{\longrightarrow} \mathrm{E}_{5 / 2 u} \oplus \mathrm{F}_{3 / 2 u} \\
& \mathrm{~F}_{3 / 2 g} \underset{2 \mathrm{pXAS}}{\stackrel{\otimes \mathrm{T}_{1 u}}{\longrightarrow}} \mathrm{E}_{1 / 2 u} \oplus 2 \mathrm{~F}_{3 / 2 u} \oplus \mathrm{E}_{5 / 2 u} .
\end{aligned}
$$

It becomes obvious that the selection rules enable to reach all three final state IRREPs in both cases: $2 p$ XAS and $1 \mathrm{~s} 2 \mathrm{p}$ RIXS. This is summarised in the combined term scheme shown in Figure 22. 

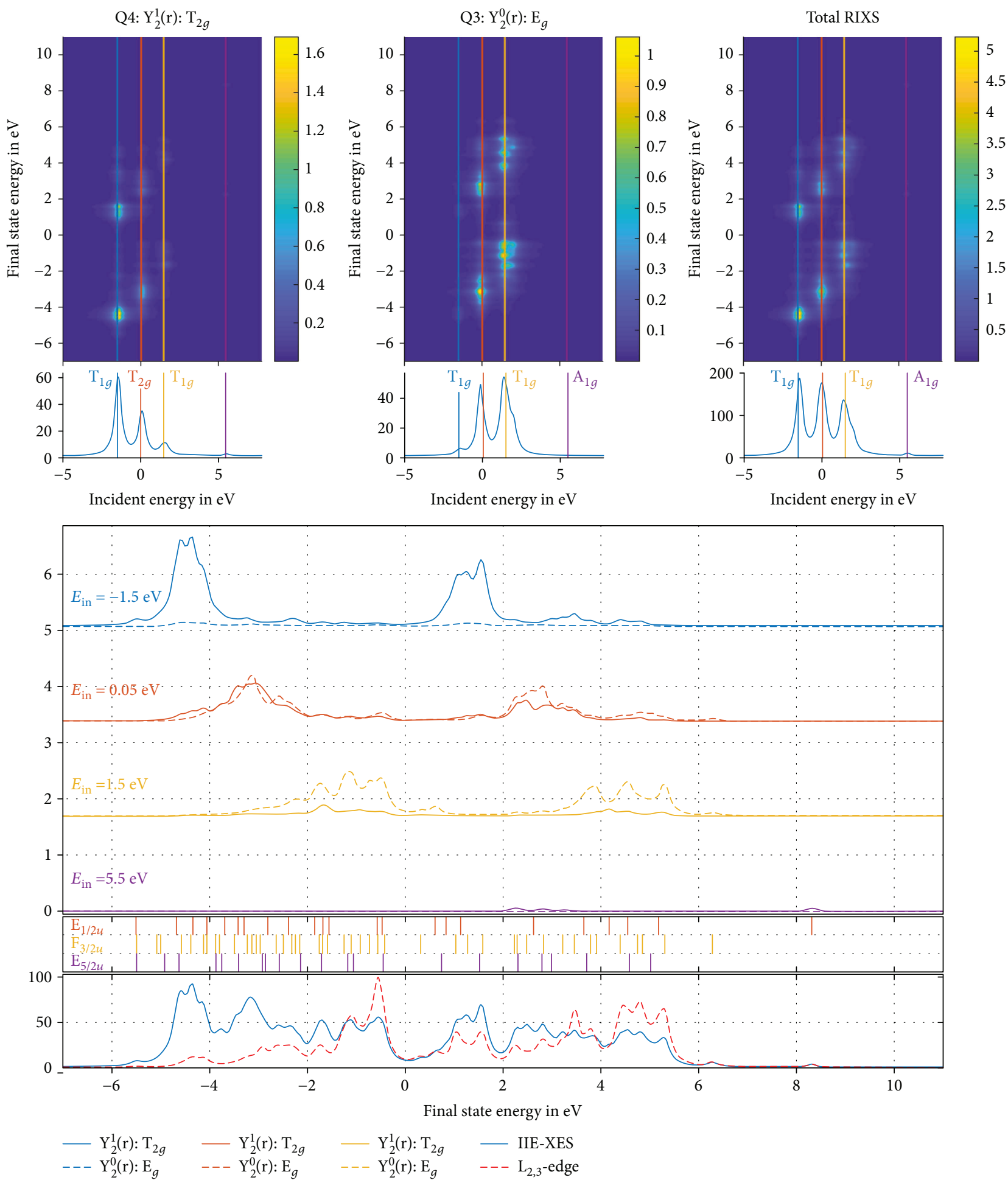

FIGURE 23: Calculated 1s2p RIXS spectrum for $\mathrm{Ti}^{3+}\left(3 \mathrm{~d}^{1}\right)$ for $10 \mathrm{D} q=1.5 \mathrm{eV}$ shown together with some selected CIE slices. The stick spectrum at the bottom illustrates the distribution of the three final state terms $\mathrm{E}_{1 / 2 u}, \mathrm{~F}_{3 / 2 u}$, and $\mathrm{E}_{5 / 2 u}$ with respect to the emissions.

Although it seems that all the final state symmetries can be reached in both cases, $2 p$ XAS and the $2 p$ XES in 1 s $2 p$ RIXS, the calculated spectra shown in Figure 23 demonstrate that the RIXS intermediate state enables to select some specific final states.
According to the combined term scheme in Figure 22, the intermediate states $\mathrm{T}_{1 g}, \mathrm{~T}_{2 g}$, and $\mathrm{E}_{g}$ enable to reach all final state symmetries. The calculated 1s2p RIXS together with some CIE slices is shown in Figure 23. However, due to the first term ${ }^{2} \mathrm{~T}_{1 g}$ corresponding to the excitation of the $1 \mathrm{~s}$ 
electron into the $t_{2 g}$ orbitals, the final states probed resonantly with RIXS are mainly the lower energy part of the states, corresponding to the " $t_{2 g}$ " part of the spectra. The intensity arises mainly from the $\mathrm{T}_{2 g}$ absorption operator. The ${ }^{2} \mathrm{~T}_{2 g}$ intermediate state selects higher energy final states equally probed by the $\mathrm{T}_{2 g}$ and $\mathrm{E}_{g} 1 \mathrm{~s}$ XAS operators.

The second term ${ }^{2} \mathrm{~T}_{1 g}$ (from ${ }^{2} \mathrm{P}$ atomic term symbol) corresponds to the absorption to the $e_{g}$ orbitals and is consequently probed by the $\mathrm{E}_{g}$ absorption operator and corresponds to the " $e_{g}$ " part of the final states. The intermediate state ${ }^{2} \mathrm{~A}_{1 g}$ (from ${ }^{2} \mathrm{~S}$ atomic term symbol) only gives a very weak absorption via the $\mathrm{T}_{2 g}$ quadrupole operator in agreement with the selection rules as given in (37).

This intermediate state only decays to $\mathrm{E}_{1 / 2 u}$ (see, e.g., the final state peak at $E_{T} \approx 8.3 \mathrm{eV}$ in Figure 23) and $\mathrm{F}_{3 / 2 \mathrm{~g}}$ (at $E_{T} \approx 2-3 \mathrm{eV}$ ). Such intermediate state selection is identical for the $L_{3^{-}}$and $L_{2}$-edges. This is similar to the results obtained for $\mathrm{Ti}^{4+}$ where the " $t_{2 g}$ " and " $e_{g}$ " parts of the finals states are selected with the intermediate state of RIXS.

5.1.1. 1s2p RIXS Experiments of $3 d^{1}$ Systems. Experimental $1 \mathrm{~s} 2 \mathrm{p}$ RIXS spectra of $3 \mathrm{~d}^{1} \mathrm{~V}^{4+}$ ions in $\mathrm{TiO}_{2}$ have been measured by Bordage [41]. However, the experimental resolution does not allow to compare the data in detail with the calculations as shown in Figure 23.

5.2. $3 d^{2}$ Ground State System, for Example, $\mathrm{Cr}^{4+}$. In this part, we present the elementary differences between $2 p$ XAS and 1s2p RIXS for the case of tetravalent chromium $\left(\mathrm{Cr}^{4+}\right)$. First, we gather the relevant information analogue to the previous cases which are then summarised in a combining term scheme illustrating the transition paths in 1s $2 p$ RIXS (1s XAS, 2p XES) and the direct 2p XAS. The electronic configurations involved here are

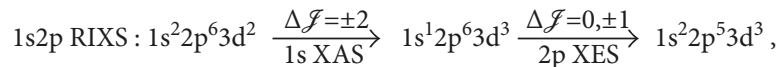

$$
\begin{aligned}
& 2 \mathrm{p} \mathrm{XAS}: 1 \mathrm{~s}^{2} 2 \mathrm{p}^{6} 3 \mathrm{~d}^{2} \stackrel{\Delta \mathscr{J}=0, \pm 1}{2 \mathrm{pXAS}} \longrightarrow 1 \mathrm{~s}^{2} 2 \mathrm{p}^{5} 3 \mathrm{~d}^{3} .
\end{aligned}
$$

In the following, we will discuss this case with respect to the influence of the SOC in the intermediate state leading to a significant increase of the possible 1s 2 p RIXS final states. The atomic case will not be detailed, and we will only discuss the influence of the $\mathrm{O}_{h}$ crystal field for $10 \mathrm{D} q=2.1 \mathrm{eV}$.

The initial state electron configuration $1 s^{2} 2 \mathrm{p}^{6} 3 \mathrm{~d}^{2}$ corresponds to the atomic ground state term ${ }^{3} \mathrm{~F}\left(\mathrm{O}_{3}\right)$ with 45 microstates. It translates into an $\mathrm{O}_{h}$ crystal field (without $3 \mathrm{~d}$ SOC) into a ${ }^{3} \mathrm{~T}_{1 g}$ ground state symmetry with 9 microstates. By including $3 \mathrm{~d}$ SOC $\zeta_{3 \mathrm{~d}}$, we find the further splittings of the ${ }^{3} \mathrm{~T}_{1 g}$ ground state term $\left(S=1 \rightarrow \otimes \mathrm{T}_{1 g}\right)$ :

$$
{ }^{3} \mathrm{~F}_{4}\left(\mathrm{O}_{3}\right) \stackrel{\mathrm{CF}}{\longrightarrow}{ }^{3} \mathrm{~T}_{1 g}\left(\mathrm{O}_{h}\right) \stackrel{\mathrm{SOC}}{\longrightarrow} \mathrm{A}_{1 g} \oplus \mathrm{E}_{g} \oplus \mathrm{T}_{1 g} \oplus \mathrm{T}_{2 g}\left(\mathrm{O}_{h}\right) .
$$

From these terms, $\mathrm{E}_{g}$ lies lowest in energy; hence, the ground state is ${ }^{3} \mathrm{~T}_{1 g}\left[\mathrm{E}_{g}\right]\left(\mathrm{O}_{h}\right)$.

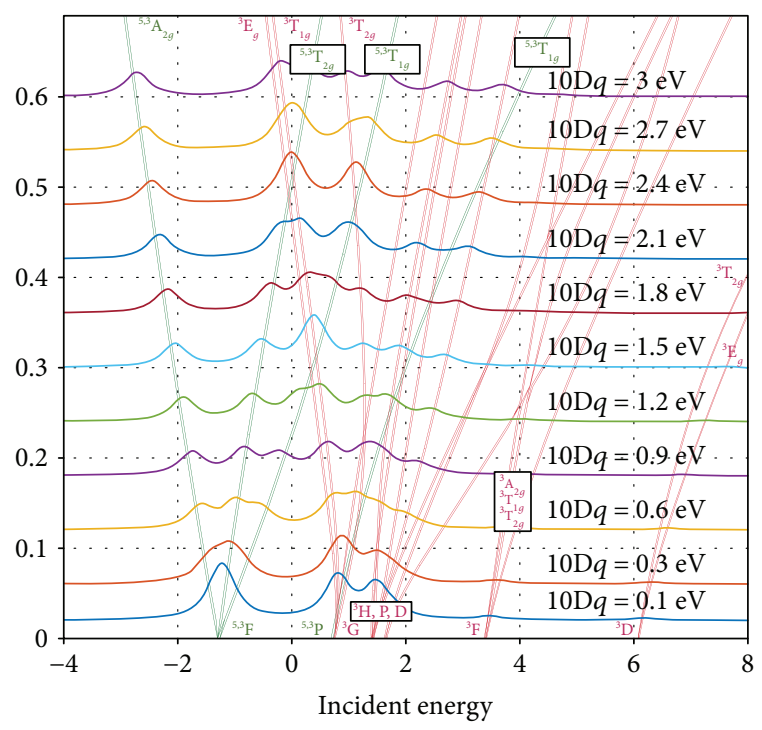

FIGURE 24: Effect of the $\mathrm{O}_{h}$ crystal field on the 1s XAS projections of the calculated 1s2p RIXS spectra for $\mathrm{Cr}^{4+}\left(3 \mathrm{~d}^{2}\right)$. The corresponding ELD overlay illustrates the distribution of the reachable intermediate state terms contributing in 1s XAS.

The intermediate state configuration $1 s^{1} 3 \mathrm{~d}^{3}$ with 2 . $120=240$ microstates translates into the atomic term symbols ${ }^{3,5} \mathrm{~F},{ }^{3,5} \mathrm{P},{ }^{3} \mathrm{H},{ }^{3} \mathrm{G},{ }^{3} \mathrm{D}$, and ${ }^{3} \mathrm{P}$ (Table 1 ). Using Table 2 one finds the branchings of the atomic states in an $\mathrm{O}_{h}$ crystal field. The resulting $\mathrm{O}_{h}$ term symbols are ${ }^{3,5} \mathrm{~A}_{2 g},{ }^{3,5} \mathrm{~T}_{2 g},{ }^{3,5} \mathrm{~T}_{1 g}$, ${ }^{3} \mathrm{~A}_{1 g},{ }^{3} \mathrm{~A}_{2 g},{ }^{3} \mathrm{E}_{g},{ }^{3} \mathrm{~T}_{1 g}$, and ${ }^{3} \mathrm{~T}_{2 g}$. However, the Coulomb exchange $G_{\text {sd }}$ is small; hence, the splitting between the triplet and the quintet spin states is small and beyond the accessible energy resolution. The energy splittings of those terms are qualitatively similar to the Tanabe-Sugano diagram of a $3 \mathrm{~d}^{3}$ electron configuration.

Because the $3 \mathrm{~d}$ SOC is weak, the spin is conserved leading to the 1s XAS transitions being dominated by transitions into the spin triplet states, while the matrix elements of the transition to the spin quintet terms are weak. Thus, we will focus on the spin triplet symmetries.

$$
\begin{aligned}
& { }^{3} \mathrm{~T}_{1 g} \stackrel{{ }^{\otimes \mathrm{T}_{2 g}}}{\longrightarrow}{ }^{3} \mathrm{~A}_{2 g} \oplus^{3} \mathrm{E}_{g} \oplus^{3} \mathrm{~T}_{1 g} \oplus^{3} \mathrm{~T}_{2 g}, \\
& { }^{3} \mathrm{~T}_{1 g} \underset{\text { 1s XAS }}{\stackrel{\otimes \mathrm{E}_{g}}{\longrightarrow} \underbrace{3}_{\text {Accessible IS terms }} \mathrm{T}_{1 g} \oplus^{3} \mathrm{~T}_{2 g}} .
\end{aligned}
$$

As can be seen from the results found in (43) and (44), starting from a ${ }^{3} \mathrm{~T}_{1 g}$ ground state, all but the ${ }^{3} \mathrm{~A}_{1 g}$ intermediate state symmetries can be reached via the quadrupole 1s XAS. The 1s XAS projections of the $1 \mathrm{~s} 2 \mathrm{p}$ RIXS for $10 \mathrm{D} q$ ranging from $0.1 \mathrm{eV}$ to $3.0 \mathrm{eV}$ are shown in Figure 24.

For the $2 \mathrm{p}$ XES decays in $1 \mathrm{~s} 2 \mathrm{p}$ RIXS, however, the inclusion of SOC is again required. Using the $\mathrm{T}_{1 g}$ IRREP $\left(S=1 \rightarrow \otimes \mathrm{T}_{1 g}\right)$ and the direct product table (Table 3 ), one finds the intermediate state symmetries including $3 \mathrm{~d}$ 


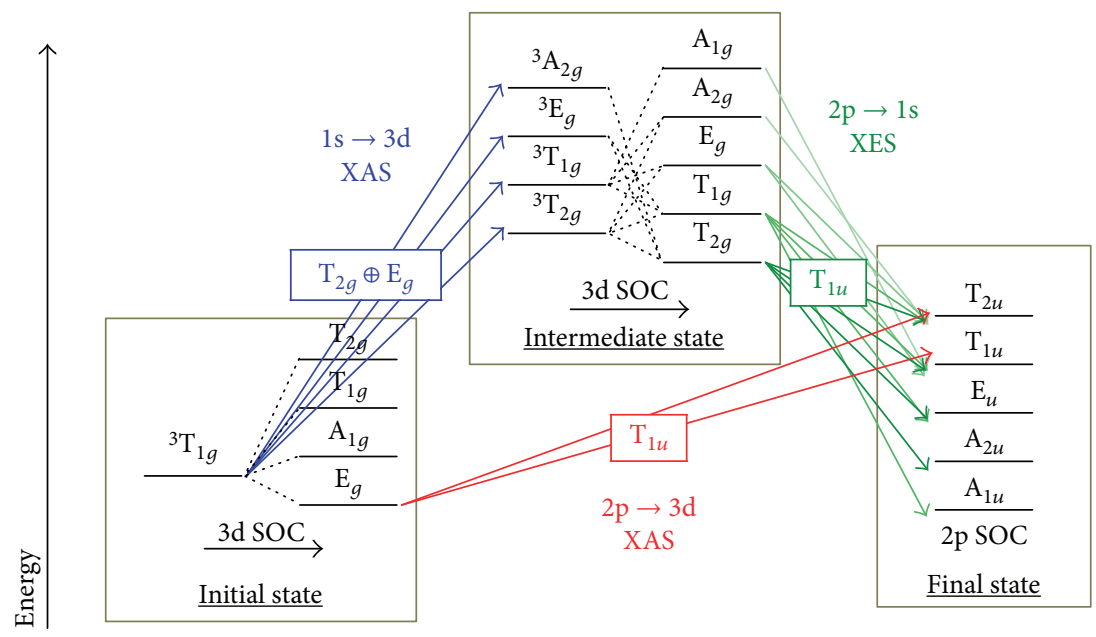

FIGURE 25: Multielectronic term scheme for $\mathrm{Cr}^{4+}\left(3 \mathrm{~d}^{2}\right)$ comparing the $1 \mathrm{~s} 2 \mathrm{p}$ RIXS and direct $2 \mathrm{p}$ XAS pathways. Where SOC is included, the spin multiplicity is omitted as its meaning is lost in that case.

SOC $\zeta_{3 \mathrm{~d}}$ as also shown in the combined term scheme in Figure 25.

$$
\begin{aligned}
& { }^{3} \mathrm{~A}_{2 g} \otimes \mathrm{T}_{1 g}=\mathrm{T}_{2 g}, \\
& { }^{3} \mathrm{E}_{g} \otimes \mathrm{T}_{1 g}=\mathrm{T}_{1 g} \oplus \mathrm{T}_{2 g}, \\
& { }^{3} \mathrm{~T}_{1 g} \otimes \mathrm{T}_{1 g}=\mathrm{A}_{1 g} \oplus \mathrm{E}_{g} \oplus \mathrm{T}_{1 g} \oplus \mathrm{T}_{2 g}, \\
& { }^{3} \mathrm{~T}_{2 g} \otimes \mathrm{T}_{1 g}=\mathrm{A}_{2 g} \oplus \mathrm{E}_{g} \oplus \mathrm{T}_{1 g} \oplus \mathrm{T}_{2 g} .
\end{aligned}
$$

The subsequent $2 \mathrm{p}$ XES starts from any of the intermediate state symmetries found in expression (45). The possible $2 \mathrm{p}$ XES final state symmetries are all even symmetries as given in expressions (28), (29), (30), (31), and (32).

The final state $\left(1 \mathrm{~s}^{2} 2 \mathrm{p}^{5} 3 \mathrm{~d}^{3}\right)$ contains $6 \cdot 120=720$ microstates due to the hole in the 2 p shell spanning $\mathscr{J}$ from 0 to 7 . In an $\mathrm{O}_{h}$ crystal field, these terms branch into the five total symmetry IRREPs $\mathrm{A}_{1 u}, \mathrm{~A}_{2 u}, \mathrm{E}_{u}, \mathrm{~T}_{1 u}$, and $\mathrm{T}_{2 u}$. In other words, all existing FS symmetries can be reached in the $2 p$ XES.

Also for 2p XAS, spin-orbit interaction is crucial. Thus, starting from the ${ }^{3} \mathrm{~T}_{1 g}\left[\mathrm{E}_{g}\right]\left(\mathrm{O}_{\mathrm{h}}\right)$ ground state and using the $\mathrm{T}_{1 u}$ IRREP, we find that only $\mathrm{T}_{1 u}$ and $\mathrm{T}_{2 u}$ IRREPs are reached, which makes a drastic difference between $2 p$ XAS and $1 \mathrm{~s} 2 \mathrm{p}$ RIXS. The selection rules for a $3 \mathrm{~d}^{2}$ ground state system are summarised in Figure 25 showing all reachable symmetries.

According to the electric quadrupole selection rules derived in (43), the $\mathrm{A}_{2 g}$ and $\mathrm{E}_{g}$ intermediate state symmetries are only reachable via the quadrupole $\mathrm{T}_{2 g}$ IRREP. This is confirmed in Figure 26 (RIXS maps) where the low energy peak at $E_{\text {in }}=-$ $2.25 \mathrm{eV}$ appears only in the RIXS map corresponding to the $\mathrm{T}_{2 g}$ IRREP. The $t_{2 g}$ and $e_{g}$ components appear well separated with (i) the $t_{2 g}$ components probed by the $\mathrm{T}_{2 g}$ absorption giving two intense and narrow peaks at $E_{T} \approx-6.5 \mathrm{eV}$ and (ii) the $e_{g}$ components probed by the $\mathrm{E}_{g}$ absorption leading to a larger group of final states spread in energy between $E_{T}=-5 \mathrm{eV}$ and $0 \mathrm{eV}$. Furthermore, it can be noted that the X-ray emission spectra $(2 \mathrm{p}$ XES) are here overall very rich. The corresponding sticks in Figure 26 illustrate how dense the reachable final states are distributed. We observe that the $2 \mathrm{p}$ XAS and 1s $2 \mathrm{p}$ RIXS differs mainly in the low energy final states: the 1s2p RIXS enhances the final states below $E_{T} \approx-2.5 \mathrm{eV}$ to which the $2 \mathrm{p}$ XAS transition matrix elements are weak. We note that the $1 \mathrm{~s} 2 \mathrm{p}$ RIXS spectra which are the closest to the $2 \mathrm{p}$ XAS spectrum are those obtained at the higher energy transfers at $E_{T}>0 \mathrm{eV}$.

Considering now the quantitative influence of the natural broadening as the principal limit of any experimental spectrum, it becomes clear that an experimental separation of the final states is impossible. However, high-resolution RIXS measurements enable to extract CIE slices similar to the illustrated examples shown in Figure 26.

And finally, in Figure 27, we show the 2p XES projections of the calculated $1 s 2 p$ RIXS to illustrate its evolution when the crystal field value $10 \mathrm{D} q$ is scaled from $10 \mathrm{D} q=$ $0.1 \mathrm{eV}, \ldots, 3 \mathrm{eV}$.

5.2.1. $1 s 2 p$ RIXS Experiments of $3 d^{2}$ Systems. Experimental $1 \mathrm{~s} 2 \mathrm{p}$ RIXS spectra of $3 \mathrm{~d}^{2} \mathrm{CrO}_{2}$ have been published by Zimmermann et al. [9]. Analysis of the magnetic circular dichroism (MCD) of the $1 \mathrm{~s} 2 \mathrm{p}$ RIXS revealed that the preedge structure that is visible in the $1 \mathrm{~s} 2 \mathrm{p}$ RIXS plane is dominated by a nonlocal contribution. The MCD data reveals the real quadrupole peak, but due to its low intensity and limited resolution, it does not allow to compare the quadrupole peak to the spectra calculated in Figure 26.

5.3. $3 d^{3}$ Ground State System, for Example, $\mathrm{Cr}^{3+}$. The electronic configurations involved for a $3 \mathrm{~d}^{3}$ ion are summarised below:

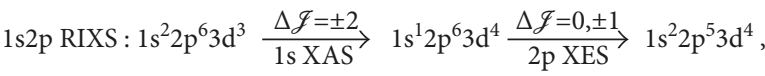

$$
\begin{aligned}
& \text { 2p XAS : } 1 \mathrm{~s}^{2} 2 \mathrm{p}^{6} 3 \mathrm{~d}^{3} \longrightarrow \frac{\Delta \mathscr{J}=0, \pm 1}{2 \mathrm{pXAS}} \longrightarrow 1 \mathrm{~s}^{2} 2 \mathrm{p}^{5} 3 \mathrm{~d}^{4} \text {. }
\end{aligned}
$$



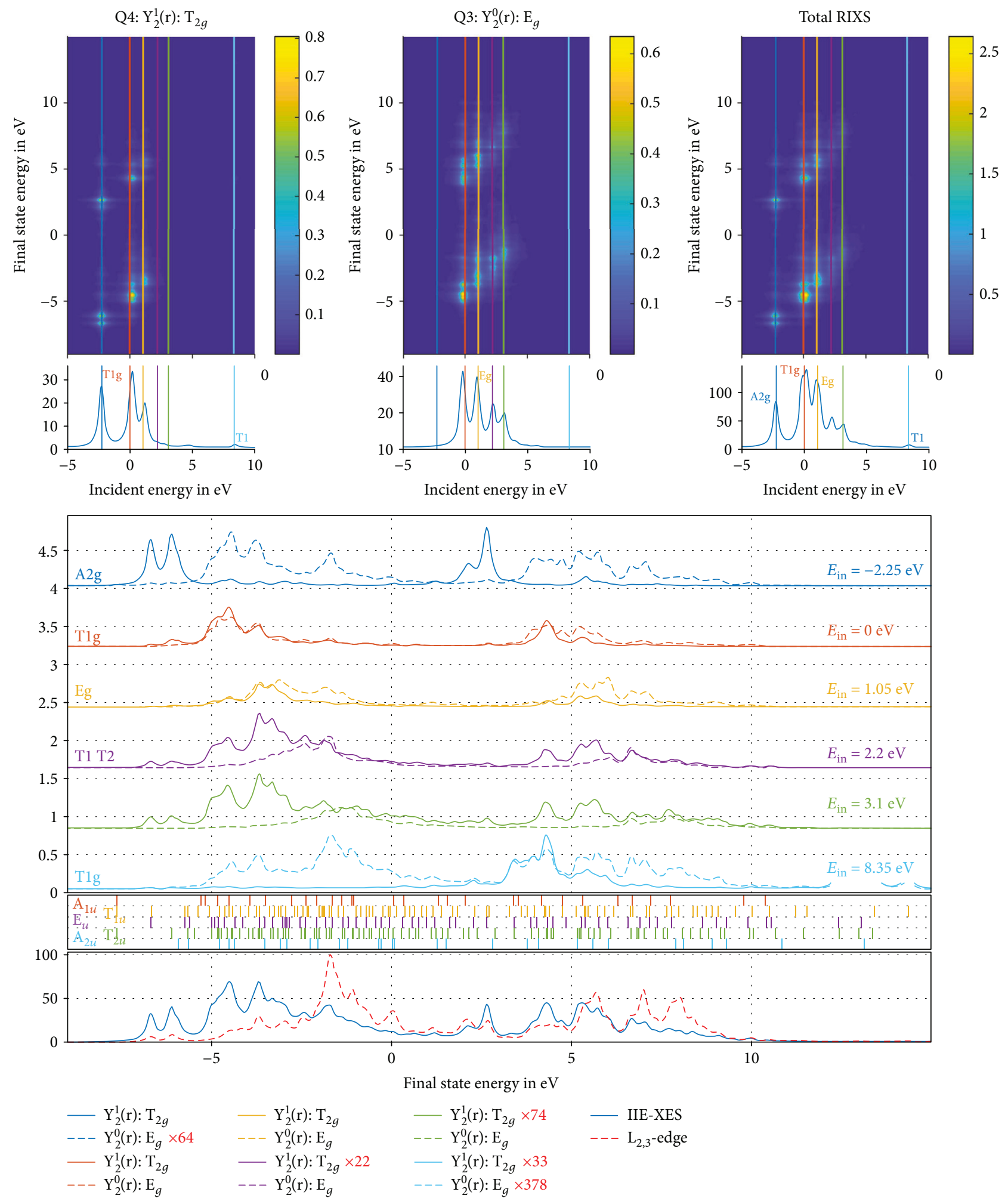

FIgURE 26: Calculated 1s2p RIXS spectrum for $\mathrm{Cr}^{4+}\left(3 \mathrm{~d}^{2}\right)$ for $10 \mathrm{D} q=2.1 \mathrm{eV}$. Note that the $\mathrm{A}_{2 g}$ intermediate state symmetry is in agreement with the selection rules only populated via the $\mathrm{T}_{2 g} 1 \mathrm{~s}$ XAS IRREP. The slices have been scaled to a comparable intensity to enhance their character better. The bottom plot compares the integrated incident energy (IIE) $2 p$ XES with the 2p XAS ( $\mathrm{L}_{2,3}$-edge). 


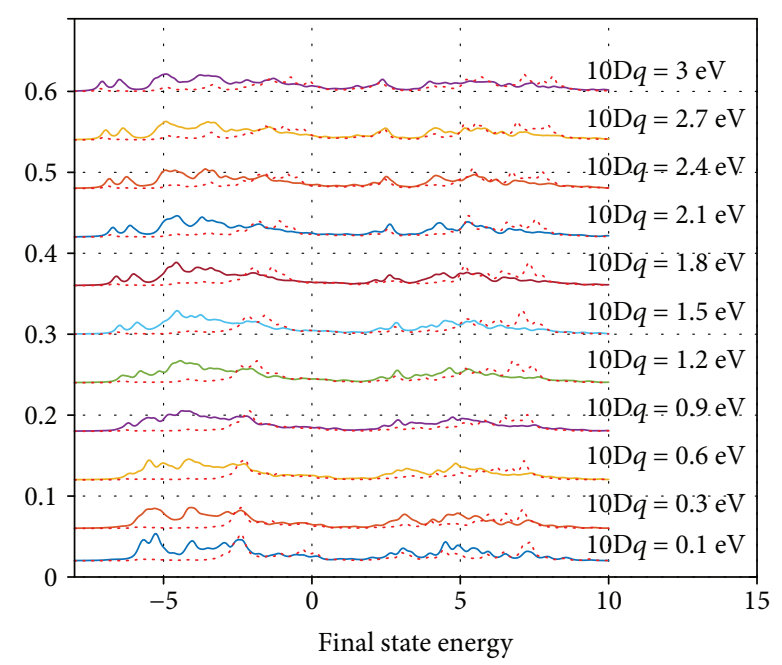

FIGURE 27: Effect of the $\mathrm{O}_{h}$ crystal field on the 2p XES projections of the calculated 1s2p RIXS spectra for $\mathrm{Cr}^{4+}\left(3 \mathrm{~d}^{2}\right)$.

For $3 \mathrm{~d}^{3}$ systems such as $\mathrm{Cr}^{3+}$, the initial state configuration $1 \mathrm{~s}^{2} 2 \mathrm{p}^{6} 3 \mathrm{~d}^{3}$ (120 microstates) translates in $\mathrm{O}_{h}$ to a ${ }^{4} \mathrm{~A}_{2 g}$ ground state (4 microstates). With $3 \mathrm{~d} \operatorname{SOC}(\mathcal{\delta}=3 / 2$ $\left.\rightarrow \otimes \mathrm{F}_{3 / 2 g}\right)$, the ground state of a $3 \mathrm{~d}^{2}$ ion is thus ${ }^{4} \mathrm{~A}_{2 g}\left[\mathrm{~F}_{3 / 2 g}\right]$ $\left(\mathrm{O}_{h}\right)$ (Table 5).

The intermediate state terms (420 microstates) contain spin sextets, quartets, and doublets and orbital momentum ranging from $\mathrm{S}$ to $\mathrm{I}$. They split in the $\mathrm{O}_{h}$ crystal field as the Tanabe-Sugano diagram of $3 \mathrm{~d}^{4}$ systems. Those terms, neglecting $3 \mathrm{~d}$ spin-orbit interaction, branch into multiple $\mathrm{O}_{h}$ terms:

$$
{ }^{6,4,2} \mathrm{~T}_{2 g},{ }^{6,4,2} \mathrm{E}_{g},{ }^{4,2} \mathrm{~T}_{1 g},{ }^{4,2} \mathrm{~A}_{2 g} \text {, and }{ }^{4,2} \mathrm{~A}_{1 g}
$$

For 1s XAS, the weak spin-orbit coupling in the $3 \mathrm{~d}$ shell results in spin-conserving transitions from the ground state ${ }^{4} \mathrm{~A}_{2 g}$ to the spin-quartet intermediate states with quadrupole IRREPs ${ }^{4} \mathrm{~T}_{1 g}$ and ${ }^{4} \mathrm{E}_{g}$ :

$$
\begin{aligned}
& { }^{4} \mathrm{~A}_{2 g} \stackrel{{ }^{\otimes \mathrm{T}_{2 g}}}{\longrightarrow}{ }^{4} \mathrm{~T}_{1 g}, \\
& { }^{4} \mathrm{~A}_{2 g} \underset{1 \mathrm{sXAS}}{\stackrel{\otimes \mathrm{E}_{g}}{\longrightarrow}}{ }^{4} \mathrm{E}_{g} .
\end{aligned}
$$

This illustrates, though many symmetries exist in the intermediate state, only the transitions into the $\mathrm{T}_{1 g}$ and $\mathrm{E}_{g}$ symmetries contribute to the spectrum as can be seen in Figure 28.

Again, as detailed throughout the examples above for the $2 \mathrm{p}$ XES decays, consideration of $3 \mathrm{~d}$ SOC is again required. Hence, we give the splitting of the two reachable intermediate state terms ${ }^{4} \mathrm{~T}_{1 g}$ and ${ }^{4} \mathrm{E}_{g}$ due to $\operatorname{SOC}\left(\mathcal{S}=3 / 2 \rightarrow \otimes \mathrm{F}_{3 / 2}\right)$ :

$$
\begin{aligned}
& { }^{4} \mathrm{~T}_{1 g} \otimes \mathrm{F}_{3 / 2 g}=\mathrm{E}_{1 / 2 g} \oplus \mathrm{E}_{5 / 2 g} \oplus 2 \cdot \mathrm{F}_{3 / 2 g}, \\
& { }^{4} \mathrm{E}_{g} \otimes \mathrm{F}_{3 / 2 g}=\mathrm{E}_{1 / 2 g} \oplus \mathrm{E}_{5 / 2 g} \oplus \mathrm{F}_{3 / 2 g} .
\end{aligned}
$$

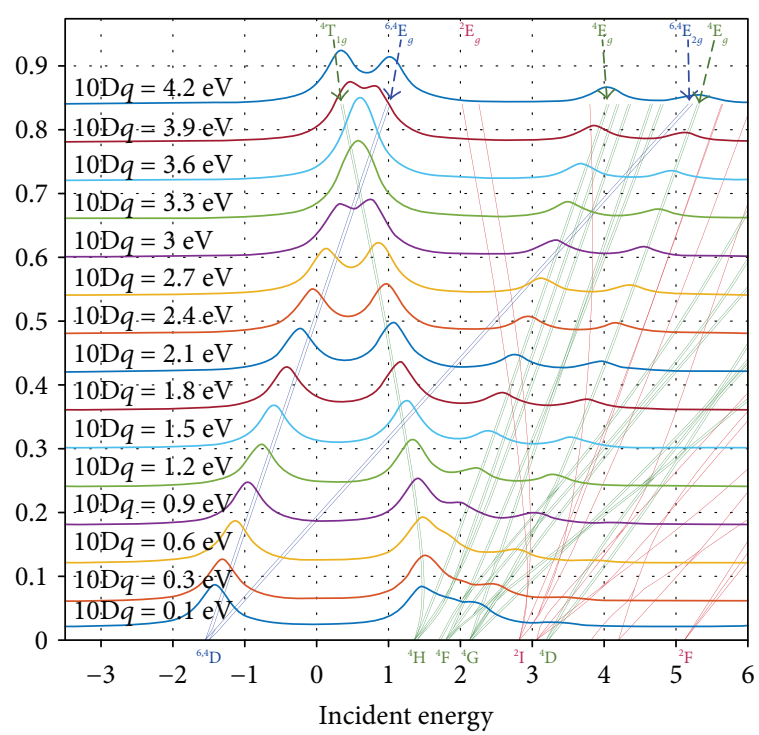

FIGURE 28: 1s XAS projections of a calculated 1s2p RIXS spectrum for $\mathrm{Cr}^{3+}\left(3 \mathrm{~d}^{3}\right)$ with ELD.

Here, it is noteworthy that both IS terms $\left({ }^{4} \mathrm{~T}_{1 g}\right.$ and $\left.{ }^{4} \mathrm{E}_{g}\right)$ are branching into the same three symmetries: $\mathrm{E}_{1 / 2}, \mathrm{E}_{5 / 2}$, and $\mathrm{F}_{3 / 2}$.

The final state $\left(1 \mathrm{~s}^{2} 2 \mathrm{p}^{5} 3 \mathrm{~d}^{4}\right)$ contains $6 \cdot 210=1260$ microstates due to the hole in the $2 \mathrm{p}$ shell. The atomic term symbols, obtained by coupling the $3 \mathrm{~d}^{4}$ atomic terms with ${ }^{2} \mathrm{P}$, span from $\mathrm{S}$ to $\mathrm{K}$ with spin doublets, quartets, and sextets and $\mathcal{F}$ values from $1 / 2$ to $15 / 2$. A detailed list can be found in the book Core Level Spectroscopy of Solids [13] (p. 103). The important information is that these $\mathscr{J}$ values branch in $\mathrm{O}_{h}$ into the three odd IRREPs $\mathrm{E}_{1 / 2 u}, \mathrm{~F}_{3 / 2 u}$, andE $\mathrm{E}_{5 / 2 u}$.

As derived in (49), the $2 \mathrm{p}$ XES decays in 1s $2 \mathrm{p}$ RIXS start from either of the three intermediate state symmetries $\mathrm{E}_{1 / 2 g}, \mathrm{E}_{5 / 2 g}$, and $\mathrm{F}_{3 / 2 g}$ and can reach $\mathrm{E}_{1 / 2 u}, \mathrm{E}_{5 / 2 u}$, and $\mathrm{F}_{3 / 2 u}$ final state IRREPs. The transitions are again given in (33), (34), and (35).

The direct 2p XAS final state terms are similar though it starts from the $\mathrm{F}_{3 / 2 g}$ ground state symmetry.

$$
\mathrm{F}_{3 / 2 g} \underset{2 \mathrm{pXAS}}{\stackrel{\otimes \mathrm{T}_{1 u}}{\longrightarrow}} \mathrm{E}_{1 / 2 u} \oplus \mathrm{E}_{5 / 2 u} \oplus 2 \mathrm{~F}_{3 / 2 u} .
$$

Altogether, the reachable final state terms are the same in $1 s 2 p$ RIXS and the direct $2 p$ XAS, the selection rules, and the corresponding transitions yield different results.

The selection rules are summarised in the term scheme shown in Figure 29.

The calculated 1s2p RIXS maps and some CIE slices together with the corresponding 1s XAS for $\mathrm{Cr}^{3+}\left(3 \mathrm{~d}^{3}\right)$ for a crystal field of $10 \mathrm{D} q=1.5 \mathrm{eV}$ are shown in Figure 30. As can be seen from the corresponding RIXS maps, from the $\mathrm{A}_{2 g}$ ground state symmetry, the quadrupole $\mathrm{T}_{2 g}$ IRREP populates only states with $\mathrm{T}_{1 g}$ symmetry, while the quadrupole $\mathrm{E}_{g}$ IRREP populates only intermediate states with $\mathrm{E}_{g}$ symmetry. The ${ }^{4} \mathrm{~T}_{1 g}\left({ }^{4} \mathrm{H}\right)$ intermediate state symmetry corresponds to the electronic configuration with four $3 \mathrm{~d}$ electrons in the $t_{2 g}$ orbitals, and as a result, the corresponding final states 


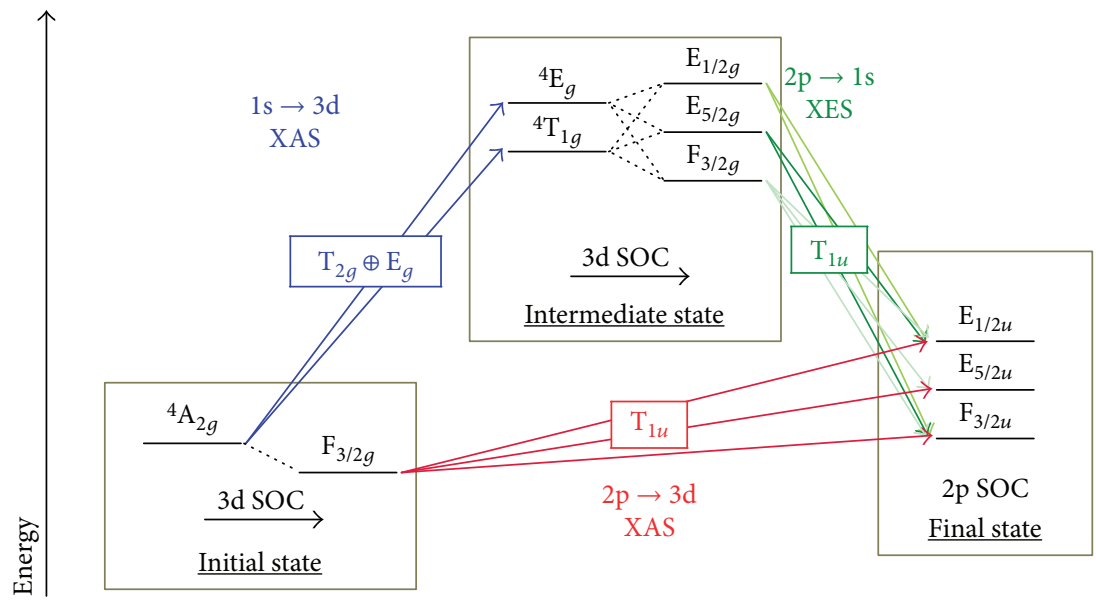

FIGURE 29: Multielectronic term scheme for $\mathrm{Cr}^{3+}\left(3 \mathrm{~d}^{3}\right)$ comparing the $1 \mathrm{~s} 2 \mathrm{p}$ RIXS and direct $2 \mathrm{p}$ XAS pathways. Where SOC is included, the spin multiplicity is omitted as its meaning is lost in that case.

(between $E_{T} \approx-5 \mathrm{eV}$ and $-2.5 \mathrm{eV}$ ) are reached in the RIXS. These states correspond to spin-quartet final states and are weak in $2 \mathrm{p}$ XAS. The first ${ }^{4} \mathrm{E}_{g}$ IRREP intermediate state $\left(E_{\text {in }} \approx-0.6 \mathrm{eV}\right.$ ) leads to the final states between $E_{T} \approx-5 \mathrm{eV}$ and $-2.5 \mathrm{eV}$, which correspond to the $e_{g}$ part of the final states with $\left(t_{2 g}\right)^{3}\left(e_{g}\right)^{1}$. The other ${ }^{4} \mathrm{E}_{g}$ intermediate state symmetries at $E_{\text {in }} \approx 2.35 \mathrm{eV}$ and $3.5 \mathrm{eV}$ lead to higher energy final states, where it is the RIXS spectrum of ${ }^{4} \mathrm{E}_{g}$ $\left(E_{T} \approx 3.5 \mathrm{eV}\right)$ that resembles more to $2 \mathrm{p}$ XAS. This is consistent with the fact that the atomic term of this intermediate state is similar to the atomic term of the ground state $\left({ }^{4} \mathrm{~F}\right)$ leading to similar 1s2p RIXS and 2p XAS spectra.

5.3.1. 1s2p RIXS Experiments of $3 d^{3}$ Systems. Examples for $3 \mathrm{~d}^{3}$ systems are $\mathrm{Cr}^{3+}$ and $\mathrm{Mn}^{4+}$. Experimental $1 \mathrm{~s} 2 \mathrm{p}$ RIXS spectra of a number of $\mathrm{Cr}^{3+}\left(3 \mathrm{~d}^{3}\right)$ systems have been published by Frommer et al. [42]. Thomas et al. (unpublished) compare $\mathrm{Cr}(\mathrm{III})(\mathrm{acac})$ and $\mathrm{Cr}_{2} \mathrm{O}_{3}$ with $1 \mathrm{~s} 2 \mathrm{p}$ RIXS crystal field multiplet calculations, equivalent to Figure 30. $\mathrm{Cr}$ (III)acac is well reproduced by the crystal field multiplet calculations. In the case of $\mathrm{Cr}_{2} \mathrm{O}_{3}$, additional intensity due to the nonlocal peak overlaps with the second pre-edge peaks in the 1s XAS. Experimental 1s2p RIXS spectra of $\mathrm{MnO}_{2}$ have been published by Glatzel et al. [43]. $\mathrm{Mn}^{4+}$ is a covalent system dominated by charge transfer. As such, the $1 \mathrm{~s} 2 \mathrm{p}$ RIXS is dominated by fluorescence and the $1 \mathrm{~s} 2 \mathrm{p}$ RIXS resonances calculated here are not visible as separate features.

5.4. $3 d^{4}$ Ground State System, for Example, $\mathrm{Mn}^{3+}$. The electronic configurations involved in this example are summarised below:

$$
\begin{aligned}
\text { 1s } 2 p \text { RIXS }: 1 s^{2} 2 p^{6} 3 d^{4} \frac{\Delta \mathscr{J}= \pm 2}{1 s \text { XAS }} 1 s^{1} 2 p^{6} 3 d^{5} \frac{\Delta \mathscr{J}=0, \pm 1}{2 p \text { XES }} 1 s^{2} 2 p^{5} 3 d^{5}, \\
2 p \text { XAS }: 1 s^{2} 2 p^{6} 3 d^{4} \underset{\Delta \mathscr{J}=0, \pm 1}{2 p \text { XAS }} 1 s^{2} 2 p^{5} 3 d^{5} .
\end{aligned}
$$

This is the first case in which the crystal field splitting energy $(10 \mathrm{D} q)$ and the pairing energy are competing. Thus, as shortly discussed in the introduction notes for Section 5, the ground state can have a high spin or low spin configuration.

5.4.1. $3 d^{4}$ System in High Spin (Quintet Ground State). The initial state electronic configuration is $1 s^{2} 3 \mathrm{~d}^{4}$ (210 microstates) having in high spin the atomic ground state term ${ }^{5} \mathrm{D}$. In the $\mathrm{O}_{h}$ crystal field, the ${ }^{5} \mathrm{D}$ ground state splits into the ${ }^{5} \mathrm{E}_{g}\left(\left|t_{2 g}^{2} e_{g}^{2}\right\rangle\right)$ and ${ }^{5} \mathrm{~T}_{2 g}\left(\left|t_{2 g}^{3} e_{g}^{1}\right\rangle\right)$ terms, where ${ }^{5} \mathrm{E}_{g}$ is the lowest in energy. The inclusion of spin-orbit interaction $\left(\mathcal{S}=2 \rightarrow \otimes\left(\mathrm{E}_{g} \oplus \mathrm{T}_{2 g}\right)\right)$ yields the following splittings in the initial state:

$$
{ }^{5} \mathrm{E}_{g} \otimes\left(\mathrm{E}_{g} \oplus \mathrm{T}_{2 g}\right)=\left(\mathrm{A}_{1 g} \oplus \mathrm{A}_{2 g} \oplus \mathrm{E}_{g}\right) \oplus\left(\mathrm{T}_{1 g} \oplus \mathrm{T}_{2 g}\right) .
$$

The ground state ${ }^{5} \mathrm{E}_{g}$ splits due to $3 \mathrm{~d}$ SOC into several symmetries from which the term $\mathrm{A}_{1 g}$ is the lowest in energy; hence, the ground state is ${ }^{5} \mathrm{E}_{g}\left[\mathrm{~A}_{1 g}\right]\left(\mathrm{O}_{h}\right)$.

The intermediate state with $1 \mathrm{~s}^{1} 3 \mathrm{~d}^{5}(2 \cdot 252=504$ microstates) corresponds to the atomic terms of $3 \mathrm{~d}^{5}$ coupled with the $1 \mathrm{~s}$ core hole. This gives spin multiplicities ranging from 1 to 7. Using Table 2, one finds the branchings of the atomic states in an $\mathrm{O}_{h}$ crystal field which are analogue to the Tanabe-Sugano diagram of $3 \mathrm{~d}^{5}$. Because the $1 \mathrm{~s}$ XAS is spin-conserving, only quintet intermediate states are reached in the absorption from the quintet high spin ground state $\left({ }^{5} \mathrm{E}_{g}\right)$. The atomic quintet terms are ${ }^{5} \mathrm{~S},{ }^{5} \mathrm{D},{ }^{5} \mathrm{~F},{ }^{5} \mathrm{G}$, and ${ }^{5} \mathrm{P}$ which correspond to the crystal field terms ${ }^{5} \mathrm{~A}_{1 g},{ }^{5} \mathrm{~A}_{2 g}$, ${ }^{5} \mathrm{E}_{g},{ }^{5} \mathrm{~T}_{1 g}$, and ${ }^{5} \mathrm{~T}_{2 g}$.

Starting from the ${ }^{5} \mathrm{E}_{g}$ ground state term, Table 3 gives the reachable intermediate state symmetries:

$$
\begin{aligned}
& { }^{5} \mathrm{E}_{g} \stackrel{\otimes \mathrm{T}_{2 g}}{\longrightarrow}{ }^{5} \mathrm{~T}_{1 g} \oplus{ }^{5} \mathrm{~T}_{2 g}, \\
& \underbrace{{ }^{5} \mathrm{E}_{g}}_{\text {GS }} \stackrel{{ }^{\otimes \mathrm{E}_{g}}}{{ }_{1 s \mathrm{XAS}}} \underbrace{{ }^{5} \mathrm{~A}_{1 g} \oplus^{5} \mathrm{~A}_{2 g} \oplus{ }^{5} \mathrm{E}_{g}}_{\text {(Accessible IS in high spin }} .
\end{aligned}
$$



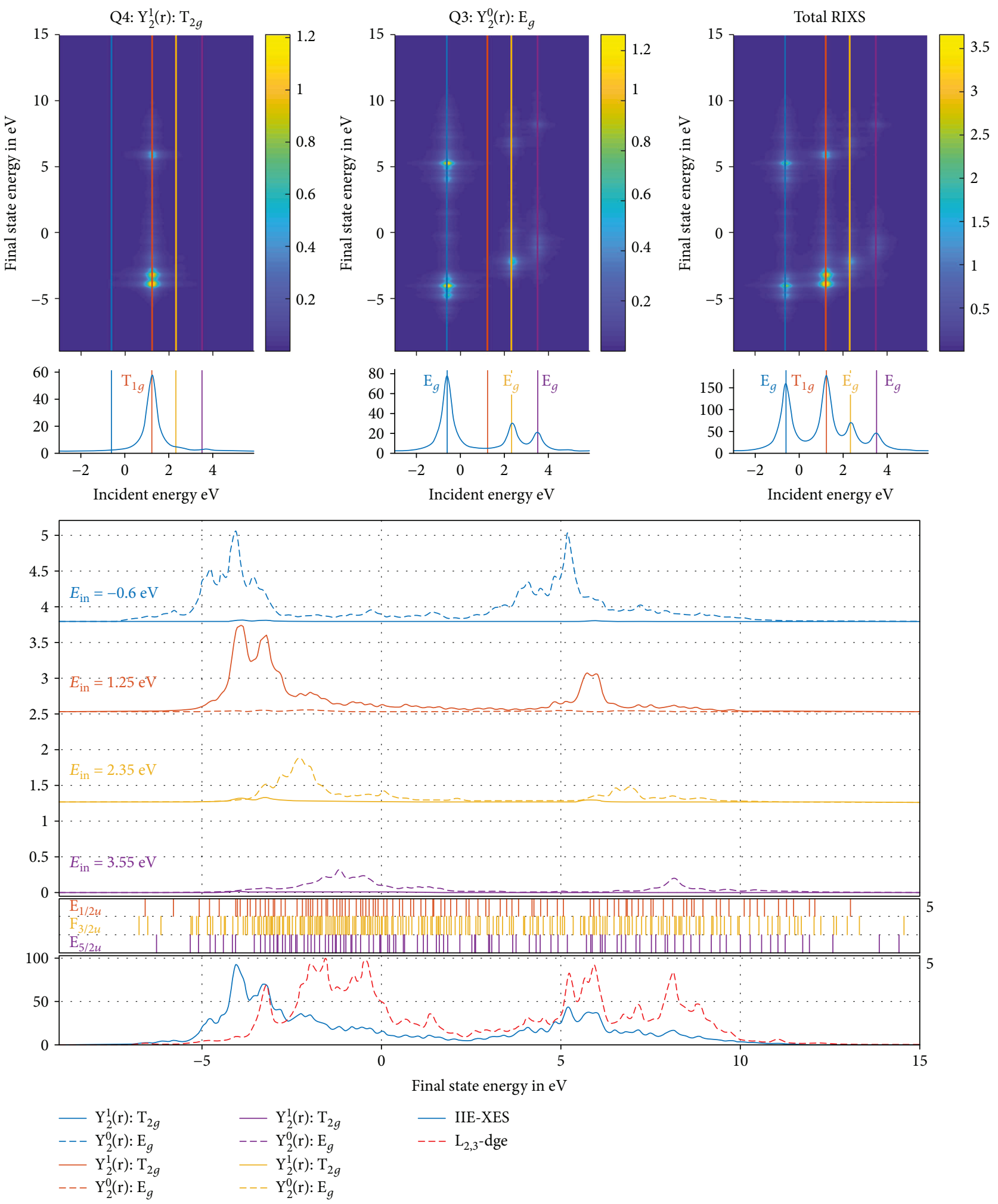

FIgURe 30: Calculated 1s2p RIXS spectrum for $3 \mathrm{~d}^{3} \mathrm{Cr}^{3+}$ for $10 \mathrm{D} q=1.5 \mathrm{eV}$.

The result is that all quintet intermediate state symmetries can be reached in 1s XAS. This is confirmed in Figure 31 where in high spin only terms with a quintet component contribute to the spectrum. Furthermore, it can be seen that the high spin configuration is maintained up to a crystal field of $10 \mathrm{D} q \approx 2.8 \mathrm{eV}$. 


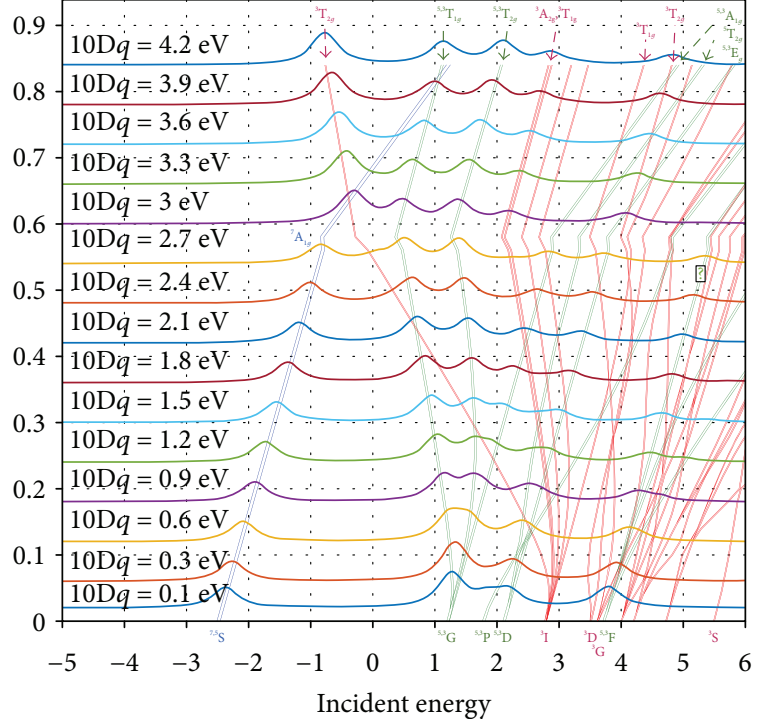

FIGURE 31: 1s XAS projection of a calculated 1s2p RIXS map for $\mathrm{Mn}^{3+}\left(3 \mathrm{~d}^{4}\right)$ with the corresponding ELD. The high spin to low spin transition at approximately $10 \mathrm{D} q \approx 2.85 \mathrm{eV}$ can be recognised. In fact, there are two HS $\rightarrow$ LS transitions: in the ground and the intermediate state.

The inclusion of the $3 \mathrm{~d}$ spin-orbit interaction $(\mathcal{S}=2 \rightarrow$ $\left.\otimes\left(\mathrm{E} \oplus \mathrm{T}_{2}\right)\right)$ yields the corresponding splittings in the intermediate state:

$$
\begin{aligned}
{ }^{5} \mathrm{~A}_{1 g} \otimes\left(\mathrm{E}_{g} \oplus \mathrm{T}_{2 g}\right) & =\left(\mathrm{E}_{g} \oplus \mathrm{T}_{2 g}\right), \\
{ }^{5} \mathrm{~A}_{2 g} \otimes\left(\mathrm{E}_{g} \oplus \mathrm{T}_{2 g}\right) & =\left(\mathrm{E}_{g} \oplus \mathrm{T}_{1 g}\right), \\
{ }^{5} \mathrm{E}_{g} \otimes\left(\mathrm{E}_{g} \oplus \mathrm{T}_{2 g}\right) & =\left(\mathrm{A}_{1 g} \oplus \mathrm{A}_{2 g} \oplus \mathrm{E}_{g}\right) \oplus\left(\mathrm{T}_{1 g} \oplus \mathrm{T}_{2 g}\right), \\
{ }^{5} \mathrm{~T}_{1 g} \otimes\left(\mathrm{E}_{g} \oplus \mathrm{T}_{2 g}\right) & =\left(\mathrm{T}_{1 g} \oplus \mathrm{T}_{2 g}\right) \oplus\left(\mathrm{A}_{2 g} \oplus \mathrm{E}_{g} \oplus \mathrm{T}_{1 g} \oplus \mathrm{T}_{2 g}\right), \\
\underbrace{{ }^{5} \mathrm{~T}_{2 g}}_{\text {IS terms }} \otimes\left(\mathrm{E}_{g} \oplus \mathrm{T}_{2 g}\right) & =\underbrace{\left(\mathrm{T}_{1 g} \oplus \mathrm{T}_{2 g}\right) \oplus\left(\mathrm{A}_{1 g} \oplus \mathrm{E}_{g} \oplus \mathrm{T}_{1 g} \oplus \mathrm{T}_{2 g}\right)}_{\text {IS terms including } 3 \mathrm{~d} \mathrm{SOC}} .
\end{aligned}
$$

Subsequently, the $2 \mathrm{p}$ XES decays can start from any of these terms.

The final state $\left(1 \mathrm{~s}^{2} 2 \mathrm{p}^{5} 3 \mathrm{~d}^{5}\right)$ contains $6 \cdot 252=1512$ microstates due to the hole in the 2 p shell spanning $\mathscr{J}$ from 0 to 8 . In an $\mathrm{O}_{h}$ crystal field, these terms branch into the five total symmetry IRREPs $\mathrm{A}_{1 u}, \mathrm{~A}_{2 u}, \mathrm{E}_{u}, \mathrm{~T}_{1 u}$, and $\mathrm{T}_{2 u}$. In other words, all even symmetries exist in the final state.

The reachable final state terms in $2 \mathrm{p}$ XES are found again with the dipole IRREP $\mathrm{T}_{1 u}$ as summarised in (28), (29), (30), (31), and (32). In short, all existing final state symmetries are also reachable in the $2 \mathrm{p}$ XES decays in $1 \mathrm{~s} 2 \mathrm{p}$ RIXS as illustrated in Figure 32.

The situation is very different for the direct $2 \mathrm{p}$ XAS. Starting from the $\mathrm{A}_{1 \mathrm{~g}}$ total symmetry IRREP term and using the $\mathrm{T}_{1 u}$ dipole IRREP, only final states with $\mathrm{T}_{1 u}$ symmetry are reachable.
The calculated 1s $2 \mathrm{p}$ RIXS, separated into the contributions via the quadrupole IRREP $\mathrm{T}_{2 g}$ and $\mathrm{E}_{g}$, together with some slices is shown in Figure 33.

A comparison of the RIXS maps in Figure 33 with the results found in expressions (53) and (54) confirms the $1 \mathrm{~s}$ XAS selection rules. As predicted in expression (53), the quadrupole IRREP $\mathrm{T}_{2 g}$ populates the intermediate state symmetries ${ }^{5} \mathrm{~T}_{1 g}$ and ${ }^{5} \mathrm{~T}_{2 g}$. While expression (54) showed for the quadrupole IRREP $\mathrm{E}_{g}$, only the intermediate state symmetries ${ }^{5} \mathrm{~A}_{1 g},{ }^{5} \mathrm{~A}_{2 g}$, and ${ }^{5} \mathrm{E}_{g}$ can be reached.

5.4.2. $3 d^{4}$ in Low Spin (Triplet Ground State). For large crystal field energies $(10 \mathrm{D} q)$, the initial state electronic configuration $1 \mathrm{~s}^{2} 3 \mathrm{~d}^{4}$ has a low spin (LS) ground state. The atomic RusselSaunders term ${ }^{3} \mathrm{D}$ translates into a ${ }^{3} \mathrm{~T}_{1 \mathrm{~g}}$ ground state with 9 microstates. The inclusion of $3 \mathrm{~d}$ SOC $\left(\mathcal{S}=1 \rightarrow \otimes \mathrm{T}_{1 g}\right)$ yields the initial state splittings:

$$
{ }^{3} \mathrm{~T}_{1 g} \otimes \mathrm{T}_{1 g}=\mathrm{A}_{1 g} \oplus \mathrm{E}_{g} \oplus \mathrm{T}_{1 g} \oplus \mathrm{T}_{2 g}
$$

The ground state ${ }^{3} \mathrm{~T}_{1 g}$ splits due to $3 \mathrm{~d}$ SOC into several symmetries from which the term $\mathrm{A}_{1 g}$ is the lowest in energy; hence, the low spin ground state is ${ }^{3} \mathrm{~T}_{1 g}\left[\mathrm{~A}_{1 g}\right]\left(\mathrm{O}_{h}\right)$.

The intermediate state electron configuration $1 s^{1} 3 \mathrm{~d}^{5}$ is the same as described above in the high spin case; however, the higher crystal field results in different energies for the intermediate state energy levels. In the low spin case, the initial state $\left({ }^{3} \mathrm{~T}_{1 g}\right)$ being a spin-triplet, the spin-conserving $1 \mathrm{~s}$ XAS only reaches spin-triplet intermediate state symmetries:

$$
\begin{aligned}
& { }^{3} \mathrm{~T}_{1 g} \stackrel{{ }^{\otimes \mathrm{T}_{2 g}}}{\longrightarrow}{ }^{3} \mathrm{~A}_{2 g} \oplus^{3} \mathrm{E}_{g} \oplus^{3} \mathrm{~T}_{1 g} \oplus^{3} \mathrm{~T}_{2 g}, \\
& \underbrace{{ }^{3} \mathrm{~T}_{1 g}}_{\text {GS }} \stackrel{{ }^{\otimes \mathrm{E}_{g}}}{\text { 1sXAS }} \underbrace{{ }^{3} \mathrm{~T}_{1 g} \oplus^{3} \mathrm{~T}_{2 g}}_{\text {(Accessible low spin IS) }} .
\end{aligned}
$$

Here, all intermediate state terms, except $\mathrm{A}_{1 g}$, are reachable in 1s XAS. The exclusion of the orbital symmetry term $\mathrm{A}_{1 g}$, due to the different ground state term, is a first notable difference with respect to the high spin case. The corresponding 1s XAS projections for the low spin case are shown in Figure 34.

Applying the $3 \mathrm{~d}$ SOC $\left(\mathcal{S}=1 \rightarrow \otimes \mathrm{T}_{1 g}\right)$ to the reachable intermediate state terms yields all even total angular momentum IRREPs.

$$
\begin{aligned}
& { }^{3} \mathrm{~A}_{2 g} \otimes \mathrm{T}_{1 g}=\mathrm{T}_{2 g}, \\
& { }^{3} \mathrm{E}_{g} \otimes \mathrm{T}_{1 g}=\mathrm{T}_{1 g} \oplus \mathrm{T}_{2 g}, \\
& { }^{3} \mathrm{~T}_{1 g} \otimes \mathrm{T}_{1 g}=\mathrm{A}_{1 g} \oplus \mathrm{E}_{g} \oplus \mathrm{T}_{1 g} \oplus \mathrm{T}_{2 g}, \\
& { }^{3} \mathrm{~T}_{2 g} \otimes \mathrm{T}_{1 g}=\mathrm{A}_{2 g} \oplus \mathrm{E}_{g} \oplus \mathrm{T}_{1 g} \oplus \mathrm{T}_{2 g} .
\end{aligned}
$$

The final states $\left(1 \mathrm{~s}^{2} 2 \mathrm{p}^{5} 3 \mathrm{~d}^{5}\right)$ are again identical to the high spin case regardless of the crystal field effects, offering the five total symmetry IRREPs $\mathrm{A}_{1 u}, \mathrm{~A}_{2 u}, \mathrm{E}_{u}, \mathrm{~T}_{1 u}$, and $\mathrm{T}_{2 u}$. 


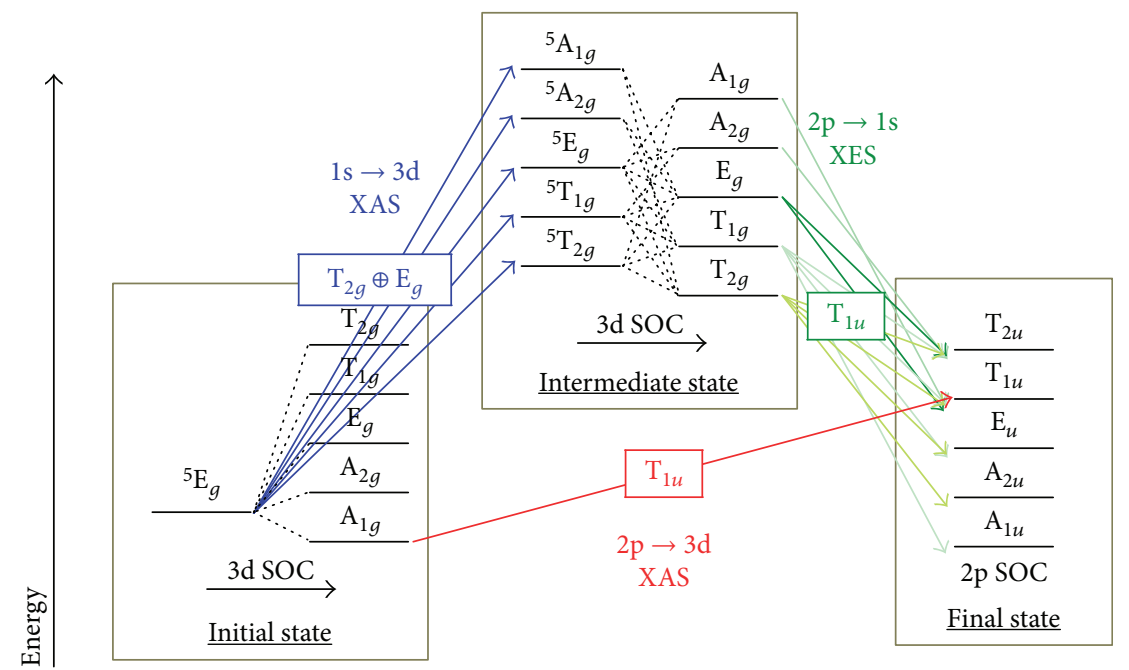

FIGURE 32: Multielectronic term scheme for $\mathrm{Mn}^{3+}\left(3 \mathrm{~d}^{4}\right)$ in high spin comparing the $1 \mathrm{~s} 2 \mathrm{p}$ RIXS and direct $2 \mathrm{p}$ XAS pathways. Where SOC is included, the spin multiplicity is omitted.

For the $2 \mathrm{p}$ XES decay, we find again from expressions (28), (29), (30), (31), and (32) that all even symmetries $\left(\mathrm{A}_{1 u}, \mathrm{~A}_{2 u}, \mathrm{E}_{u}, \mathrm{~T}_{1 u}\right.$, and $\left.\mathrm{T}_{2 u}\right)$ can be reached. In $2 \mathrm{p}$ XAS, starting from the $A_{1 g}$ total symmetry term and using the $\mathrm{T}_{1 u}$ IRREP, we find that only $\mathrm{T}_{1 u}$ final state IRREPs can be reached. The selection rules are summarised in the combined term scheme in Figure 35.

An example calculation for the low spin case $(10 \mathrm{D} q=$ $3.9 \mathrm{eV})$ of $\mathrm{Mn}^{3+}\left(3 \mathrm{~d}^{4}\right)$ is shown in Figure 36.

This example is the first case here in which the crystal field energy $(10 \mathrm{D} q)$ and the pairing energy are competing to induce a low spin configuration for sufficiently large values of $10 \mathrm{D} q$. In the present case of $\mathrm{Mn}^{3+}\left(3 \mathrm{~d}^{4}\right)$, we see the high spin to low spin transition at around $10 \mathrm{D} q \approx 2.85 \mathrm{eV}$ as is observable in the $1 \mathrm{~s}$ XAS and $2 \mathrm{p}$ XES projections (Figures 31 and 37, resp.).

One difference between the low spin and high spin $\mathrm{Mn}^{3+}$ is the spin state of the ground state. The result is that in the HS, the 1s XAS absorption reaches the spinquintet IS while in the LS case, it reaches the spin-triplet IS. This results in different final states being probed regardless of the effect of the crystal field. In the low spin case, the intermediate state is separating the two quadrupole components $\mathrm{T}_{2 g}$ and $\mathrm{E}_{g}$ (Figure 26 top row). Although the projected 1s2p RIXS and the $2 p$ XAS present close intensities across the final states, the calculations show that by choosing the 1s2p RIXS incident energy $E_{\text {in }}$, one can select specific intermediate states.

We observe that the quadrupole $\mathrm{T}_{2 g}$ IREEP can reach only one ${ }^{3} \mathrm{~T}_{2 g}\left({ }^{3} \mathrm{I}\right)$ intermediate state symmetry, while it cannot be reached with the quadrupole $\mathrm{E}_{g}$ operator. This is consistent with the corresponding electron configuration of the ${ }^{3} \mathrm{~T}_{2 g}$ IRREP in the $3 \mathrm{~d}$ shell: $\left|t_{2 g}^{5} e_{g}^{0}\right\rangle$.

The quadrupole $\mathrm{E}_{g}$ IRREP enables to reach only the intermediate state spin-triplets at higher energy. Consequently, it appears that in the case of low spin $\mathrm{Mn}^{3+}$, depending on the
RIXS intermediate states that are probed, one can enhance either the transitions into the $t_{2 g}$ orbitals of the final states or the transitions into the $e_{g}$ orbitals.

5.4.3. $1 s 2 p$ RIXS Experiments of $3 d^{4}$ Systems. Experimental $1 s 2 p$ RIXS spectra of a number of $\mathrm{Mn}^{3+}\left(3 \mathrm{~d}^{4}\right)$ systems have been published by Glatzel et al. [43] for $\mathrm{Mn}_{2} \mathrm{O}_{3}$ and also for a series of molecular complexes that serve as reference systems for photosystem II. The pre-edge 1s2p RIXS resonances can be separated well from the main peak. The 1s2p RIXS plane of $\mathrm{Mn}(\mathrm{III}) \mathrm{acac}$ [43] is similar to the calculated plane in Figure 36.

5.5. $3 d^{5}$ Ground State System, for Example, $\mathrm{Fe}^{3+}$. In this part, we present the elementary differences for the case of an $\mathrm{Fe}^{3+}$ ion $\left(3 \mathrm{~d}^{5}\right)\left(\mathrm{Mn}^{2+}\right.$ analogue). First, we gather the relevant information analogue to the previous cases which are again summarised in a combining term scheme illustrating the transition paths in 1s2p RIXS (1s XAS, 2p XES) and $2 p$ $\mathrm{XAS}$. The electronic configurations involved in this case are summarised below:

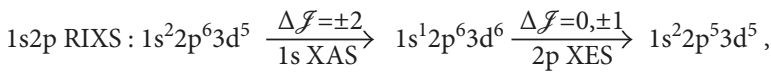

$$
\begin{aligned}
& 2 \mathrm{p} \mathrm{XAS}: 1 \mathrm{~s}^{2} 2 \mathrm{p}^{6} 3 \mathrm{~d}^{5} \stackrel{\Delta \mathcal{J}=0, \pm 1}{2 \mathrm{pXAS}} \longrightarrow 1 \mathrm{~s}^{2} 2 \mathrm{p}^{5} 3 \mathrm{~d}^{6} .
\end{aligned}
$$

Also in $3 \mathrm{~d}^{5}$ ground state systems like $\mathrm{Fe}^{3+}$ or $\mathrm{Mn}^{2+}$, high spin to low spin transitions can be observed. Thus, for large values of $10 \mathrm{D} q$, the ground state changes from ${ }^{6} \mathrm{~A}_{1 g}$ for $\left|t_{2 g}^{3} e_{g}^{2}\right\rangle$ to ${ }^{2} \mathrm{~T}_{2 g}$ for $\left|t_{2 g}^{5} e_{g}^{0}\right\rangle$.

5.5.1. $\mathrm{Fe}^{3+}\left(3 d^{5}\right)$ in High Spin (Sextet Ground State). The initial state configuration $1 \mathrm{~s}^{2} 3 \mathrm{~d}^{5}$ (252 microstates) has in high spin the ground state term ${ }^{6} \mathrm{~S}$ (Table 1) branching in $\mathrm{O}_{h}$ into $a^{6} \mathrm{~A}_{1 g}$ symmetry (6 microstates). The spin-orbit interaction 

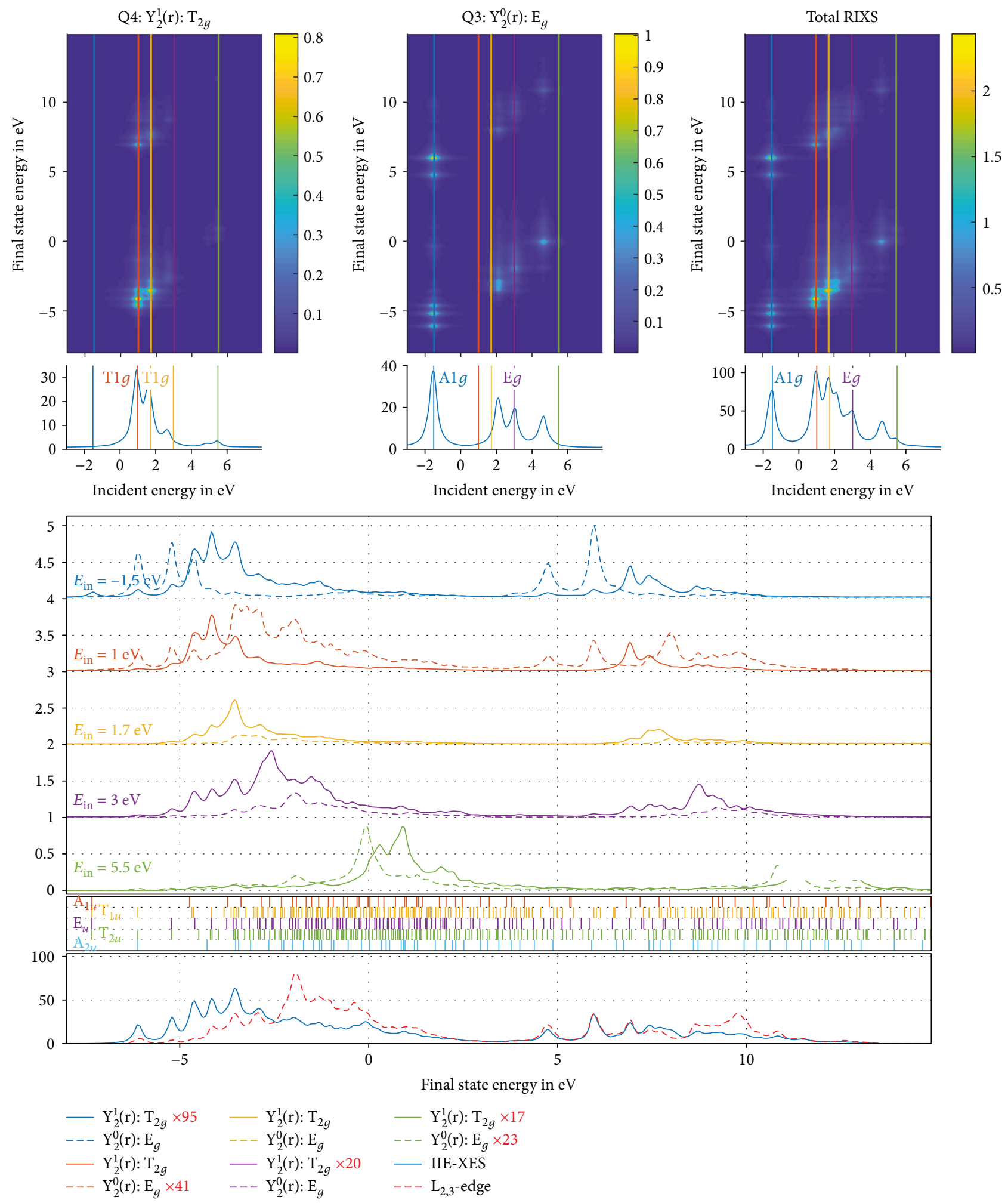

FIgURE 33: Calculated 1s2p RIXS map for $\mathrm{Mn}^{3+}\left(3 \mathrm{~d}^{4}\right)$ with a crystal field for $10 \mathrm{D} q=1.5 \mathrm{eV}$ reflecting the high spin case. As can be seen by the two RIXS maps (top row, left and middle), the contributions are in agreement with the predicted symmetries found in (51) and (52).

$\left(\mathcal{S}=5 / 2 \rightarrow \otimes\left[\mathrm{F}_{3 / 2 g} \oplus \mathrm{E}_{5 / 2 g}\right]\right)$ yields the following splittings of the ground state term:

$$
{ }^{6} \mathrm{~A}_{1 g} \otimes\left[\mathrm{F}_{3 / 2 g} \oplus \mathrm{E}_{5 / 2 g}\right]=\mathrm{F}_{3 / 2 g} \oplus \mathrm{E}_{5 / 2 g} .
$$

Here, the term $\mathrm{F}_{3 / 2 g}$ lies the lowest in energy; thus, the ground state is ${ }^{6} \mathrm{~A}_{1 g}\left[\mathrm{~F}_{3 / 2 g}\right]\left(\mathrm{O}_{h}\right)$. However, the splitting is small (few $\mathrm{meV}$ ) and thus also the $\mathrm{E}_{52 \mathrm{~g}}$ term would contribute weakly in a measurement. 


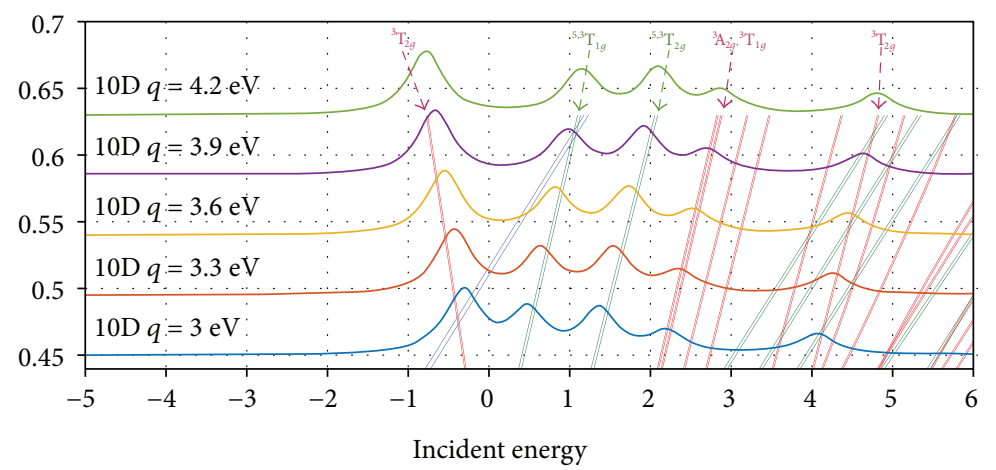

FIGURE 34: 1s XAS projection of a calculated 1s2p RIXS map for $\mathrm{Mn}^{3+}\left(3 \mathrm{~d}^{4}\right)$ in low spin.

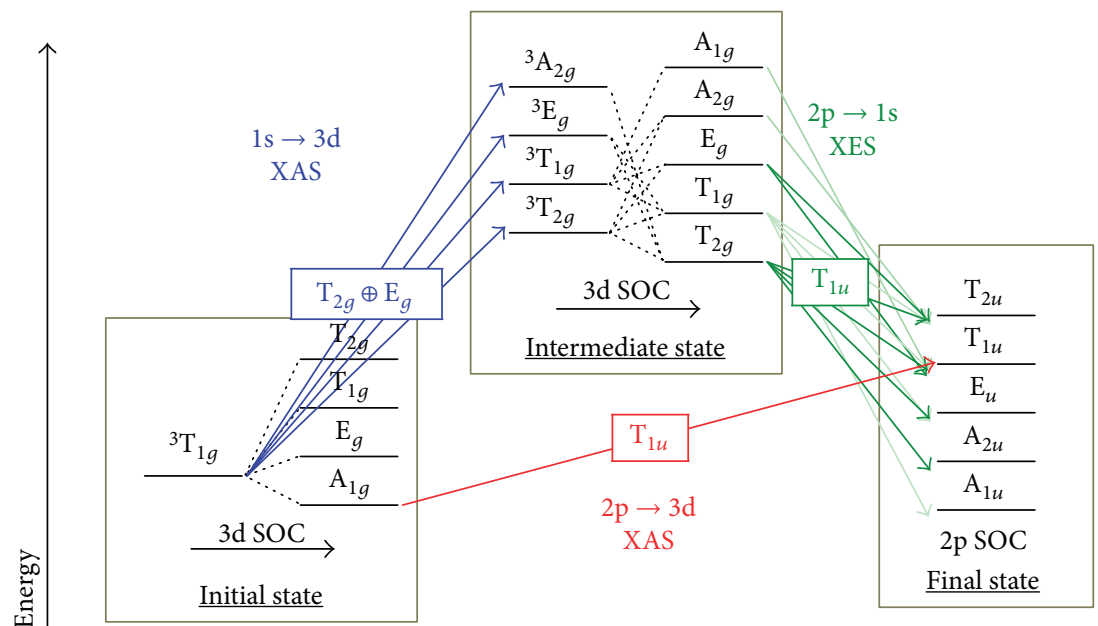

FIGURE 35: Multielectronic term scheme for $\mathrm{Mn}^{3+}\left(3 \mathrm{~d}^{4}\right)$ in low spin comparing the 1 s2 $\mathrm{p}$ RIXS and direct $2 \mathrm{p}$ XAS pathways. Where SOC is included, the spin multiplicity is omitted.

The intermediate state with $1 s^{1} 3 \mathrm{~d}^{6}(2 \cdot 210=420$ microstates) gives the atomic terms with spin multiplicity from 0 to 6 and orbital momentum from $\mathrm{S}$ to I. The $\mathrm{O}_{h}$ crystal field splits these terms analogue to the classic Tanabe-Sugano diagram of $3 \mathrm{~d}^{6}$. In $1 \mathrm{~s}$ XAS, due to the weak $3 \mathrm{~d}$ SOC starting from the ${ }^{6} \mathrm{~A}_{1 g}$ ground state, only sextet spin terms ${ }^{6} \mathrm{~T}_{2 g}$ and ${ }^{6} \mathrm{E}_{g}$ arising from the only atomic sextet spin term ${ }^{6} \mathrm{D}$ are reached (Table 3):

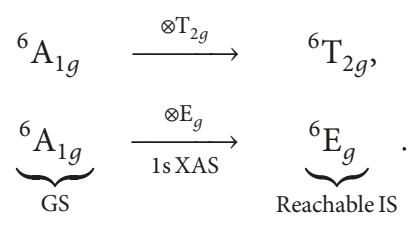

The corresponding $1 \mathrm{~s}$ XAS projections for a $3 \mathrm{~d}^{5}$ system with $10 \mathrm{D} q$ between $0.1 \mathrm{eV}$ and $4.2 \mathrm{eV}$ are shown in Figure 38. It is noteworthy that this situation presents some similarities with the case of $3 \mathrm{~d}^{0}\left(\mathrm{Ti}^{4+}\right)$. In both cases, the excited 1s electron can go into either the $t_{2 g}$ orbitals or the $e_{g}$ orbitals. The drastic difference is that for $3 \mathrm{~d}^{5}$, all spin-up orbitals are full in the total symmetric IRREP ${ }^{6} \mathrm{~A}_{1 g}\left(\mathrm{O}_{h}\right)$.
For the 2p XES decay, spin-orbit interaction is again crucial; hence, we include $3 \mathrm{~d}$ spin-orbit coupling $(\mathcal{S}=5 / 2 \rightarrow \otimes$ $\left.\left[\mathrm{F}_{3 / 2 g} \oplus \mathrm{E}_{5 / 2 g}\right]\right)$ for the reachable intermediate state terms:

$$
\begin{aligned}
{ }^{6} \mathrm{~T}_{2 g} \otimes\left[\mathrm{F}_{3 / 2 g} \oplus \mathrm{E}_{5 / 2 g}\right]= & \left(\mathrm{E}_{1 / 2 g} \oplus \mathrm{E}_{5 / 2 g} \oplus 2 \mathrm{~F}_{3 / 2 g}\right) \\
& \oplus\left(\mathrm{E}_{1 / 2 g} \oplus \mathrm{F}_{3 / 2 g}\right), \\
\underbrace{{ }^{6} \mathrm{E}_{g}}_{\text {Reachable IS }} \otimes\left[\mathrm{F}_{3 / 2 g} \oplus \mathrm{E}_{5 / 2 g}\right]= & \left(\mathrm{E}_{1 / 2 g} \oplus \mathrm{E}_{5 / 2 g} \oplus \mathrm{F}_{3 / 2 g}\right) \oplus \mathrm{F}_{3 / 2 g} .
\end{aligned}
$$

Due to the spin $(\mathcal{S}=5 / 2)$ and the resulting splittings, all three odd IRREPs $\left(\mathrm{E}_{1 / 2 g}, \mathrm{E}_{5 / 2 g}\right.$, and $\left.\mathrm{F}_{3 / 2 g}\right)$ appear more than once. Thus, the $2 p$ XES can decay from any of the three IRREPs $\mathrm{E}_{1 / 2 g}, \mathrm{E}_{5 / 2 g}$, or $\mathrm{F}_{3 / 2 g}$. As given in (33), (34), and (35), the dipole IRREP $T_{1 u}$ also enables to reach all three odd symmetries $\mathrm{E}_{1 / 2 u}, \mathrm{E}_{5 / 2 u}$, and $2 \mathrm{~F}_{3 / 2 u}$ in the final state.

The direct $2 \mathrm{p}$ XAS on the other hand starts from the $\mathrm{F}_{3 / 2 \mathrm{~g}}$ ground state term; hence, the reachable final state symmetries are identical to those found in (35). In other words, the direct $2 \mathrm{p}$ XAS reaches the same final states as the $2 \mathrm{p}$ XES decays from the $\mathrm{F}_{3 / 2 \mathrm{~g}}$ intermediate state symmetry. The resulting overall selection rules for $3 \mathrm{~d}^{5}$ in high 

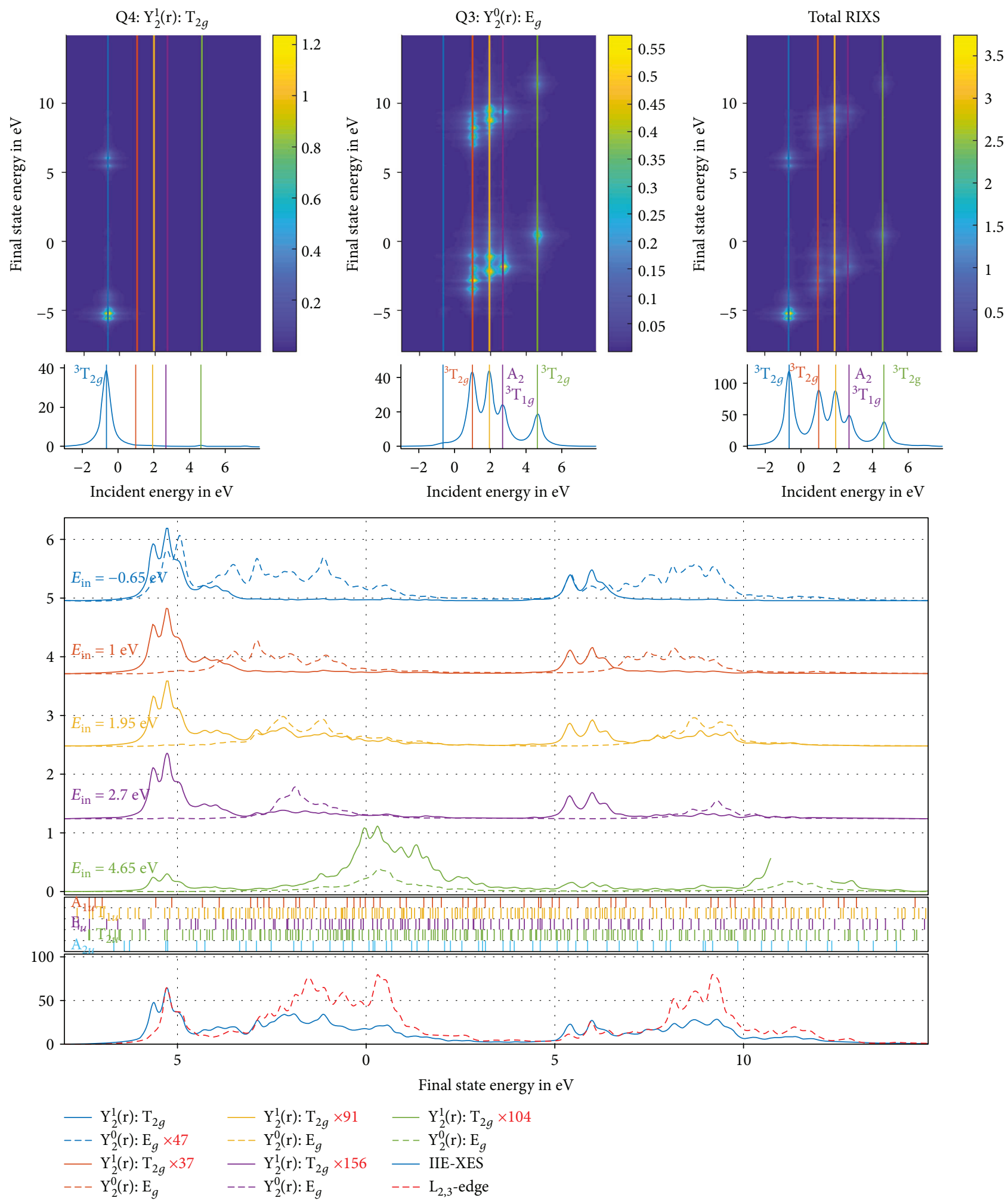

Figure 36: Calculation of 1s2p RIXS and 2p XAS for $\mathrm{Mn}^{3+}\left(3 \mathrm{~d}^{4}\right)$ for $10 \mathrm{D} q=3.9 \mathrm{eV}$ (low spin).

spin are summarised in the combined term scheme in Figure 39.

The calculated 1s2p RIXS maps shown in Figure 40 confirm the selection rules as summarised above. The two
RIXS maps corresponding to the two quadrupole IRREPs $\mathrm{T}_{2 g}$ and $\mathrm{E}_{g}$ (Figure 40 top row, left and middle) confirm that the intermediate states are probed selectively according to the quadrupole transition operator. This means that 


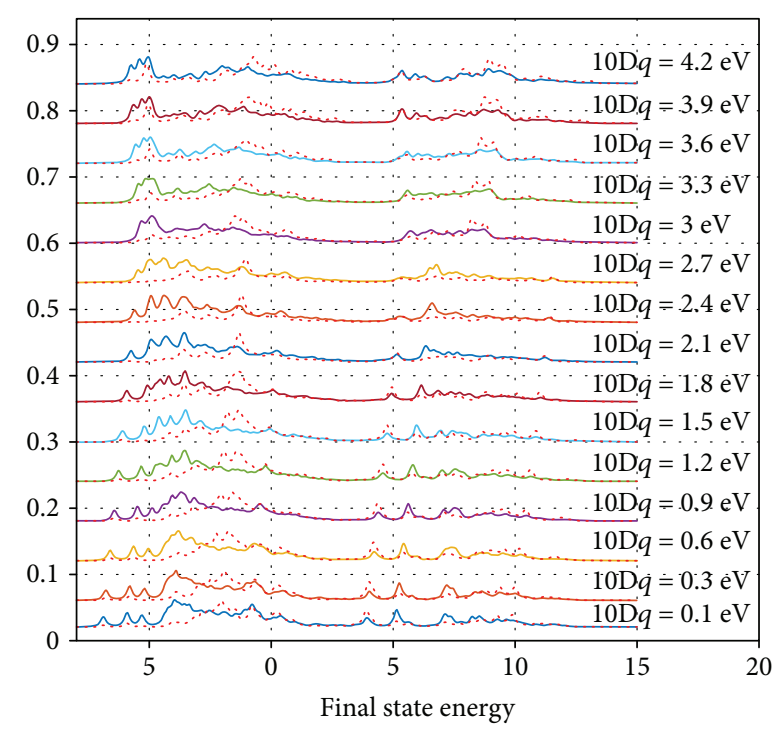

FIGURE 37: $2 \mathrm{p}$ XES projections of a calculated 1s2p RIXS spectrum for $\mathrm{Mn}^{3+}\left(3 \mathrm{~d}^{4}\right)$. Note the HS-LS transition at approximately $10 \mathrm{D} q$ $\approx 2.85 \mathrm{eV}$. In fact, there are two $\mathrm{HS} \rightarrow \mathrm{LS}$ transitions, in the GS and in the IS, which are not necessarily occurring at the same $10 \mathrm{D} q$ value.

the $e_{g}$ and $t_{2 g}$ components of the final state can, via the intermediate state, be selectively probed in $1 \mathrm{~s} 2 \mathrm{p}$ RIXS.

This example is interesting, because the 1s XAS transitions are identical to the $\mathrm{Ti}^{4+}\left(3 \mathrm{~d}^{0}\right)$ case. The ground state is totally symmetric with $\mathrm{A}_{1 g}$; thus, the quadrupole IRREPs $\mathrm{T}_{2 g}$ and $\mathrm{E}_{g}$ populate the corresponding intermediate symmetries $\left(\mathrm{T}_{2 g}\right.$ and $\left.\mathrm{E}_{g}\right)$. However, there are two important differences: (i) for the high spin configuration of $3 d^{5}$, all spin-up orbitals are full; thus, only the spin-down electrons can populate the remaining holes in the $3 \mathrm{~d}$ shell. And (ii) the final state electron configuration $2 \mathrm{p}^{5} 3 \mathrm{~d}^{6}$ of $\mathrm{Fe}^{3+}$ has, due to the six electrons in the $3 \mathrm{~d}$ orbitals, $6 \cdot 210=1260$ microstates. This translates into a very rich and dense stick spectrum shown below the slices in Figure 40.

Finally, we note that the matrix elements of the $2 p$ XAS appear very similar to those of the resonant $2 p$ XES decays occurring from the ${ }^{6} \mathrm{E}_{\mathrm{g}}$ intermediate state, while the total $2 \mathrm{p}$ XES shown as integrated incident energy (IIE) shows notable differences when compared to the $2 \mathrm{p}$ XAS due to the population from the intermediate state symmetries.

5.5.2. $\mathrm{Fe}^{3+}\left(3 d^{5}\right)$ in Low Spin (Doublet Ground State). The initial state electron configuration $3 \mathrm{~d}^{5}$ translates in low spin to $\left|t_{2 g}^{5} e_{g}^{0}\right\rangle$ with the atomic ground state term ${ }^{2} \mathrm{D}$ (10 microstates). In $\mathrm{O}_{h}$, this translates into a ${ }^{2} \mathrm{~T}_{2 g}$ ground state term with 6 microstates. The inclusion of $3 \mathrm{~d}$ spin-orbit interaction $\left(\mathcal{S}=1 / 2 \rightarrow \otimes \mathrm{E}_{1 / 2 g}\right)$ yields the following splitting:

$$
{ }^{2} \mathrm{~T}_{2 g} \otimes \mathrm{E}_{1 / 2 g}=\mathrm{E}_{5 / 2 g} \oplus \mathrm{F}_{3 / 2 g} .
$$

Here, the term $\mathrm{E}_{5 / 2 g}$ lies the lowest in energy; thus, the ground state is ${ }^{2} \mathrm{~T}_{2 g}\left[\mathrm{E}_{5 / 2 g}\right]\left(\mathrm{O}_{h}\right)$.

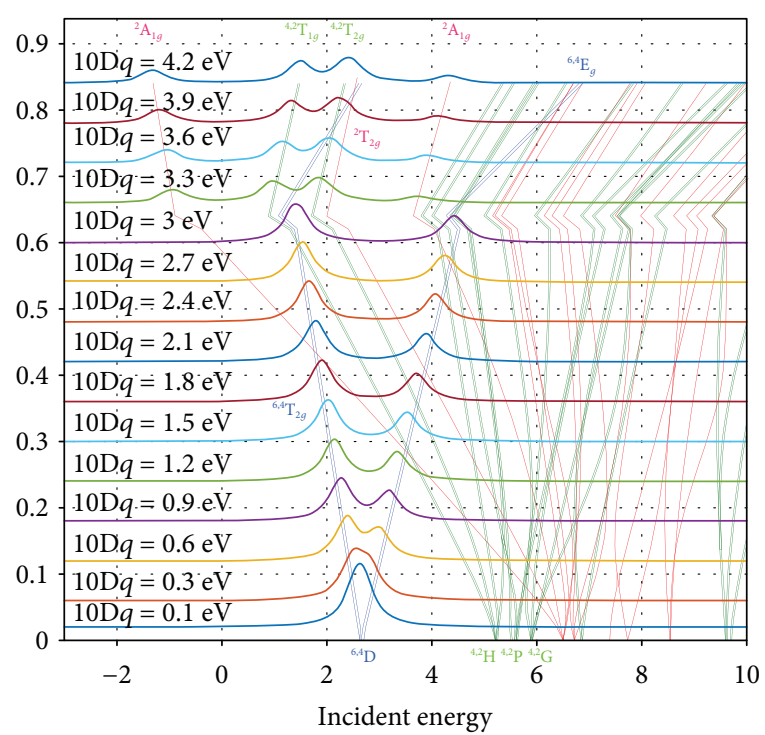

FIgURE 38: 1s XAS projections for $\mathrm{Fe}^{3+}\left(3 \mathrm{~d}^{5}\right)$ with the corresponding ELD. Notice the high spin to low spin transitions in the ground and intermediate states between $10 \mathrm{D} q \approx 3.0 \mathrm{eV}$ and $3.3 \mathrm{eV}$.

The intermediate state configuration $1 s^{1} 3 d^{6}$ is still the same as in the high spin case; however, the higher crystal field results in different energies for the intermediate state energy levels. In the low spin case, the ground state being a spin doublet $\left({ }^{2} \mathrm{~T}_{2 g}\right)$, the spin-conserving $1 \mathrm{~s}$ XAS only reaches spin doublet intermediate state IRREPs (Table 3):

$$
\begin{aligned}
& { }^{2} \mathrm{~T}_{2 g} \stackrel{\otimes \mathrm{T}_{2 g}}{\longrightarrow}{ }^{2} \mathrm{~A}_{1 g} \oplus^{2} \mathrm{E}_{g} \oplus^{2} \mathrm{~T}_{1 g} \oplus^{2} \mathrm{~T}_{2 g}, \\
& \underbrace{{ }^{2} \mathrm{~T}_{2 g}}_{\text {GS }} \stackrel{{ }^{\otimes \mathrm{E}_{g}}}{\text { 1s XAS }} \underbrace{{ }^{2} \mathrm{~T}_{1 g} \oplus^{2} \mathrm{~T}_{2 g}}_{\text {Reachable IS (low spin) }} .
\end{aligned}
$$

This means that from the ${ }^{2} \mathrm{~T}_{2 g}$ ground state the quadrupole 1s XAS, as shown in Figure 41, can reach all even intermediate state symmetries, except any $\mathrm{A}_{2 g}$ IRREPs.

The reachable intermediate state terms split due to the $3 \mathrm{~d}$ spin-orbit interaction $\left(\mathcal{S}=1 / 2 \rightarrow \otimes \mathrm{E}_{1 / 2 g}\right)$ :

$$
\begin{aligned}
& { }^{2} \mathrm{~A}_{1 g} \otimes \mathrm{E}_{1 / 2 g}=\mathrm{E}_{1 / 2 g}, \\
& { }^{2} \mathrm{E}_{g} \otimes \mathrm{E}_{1 / 2 g}=\mathrm{F}_{3 / 2 g}, \\
& { }^{2} \mathrm{~T}_{1 g} \otimes \mathrm{E}_{1 / 2 g}=\mathrm{E}_{1 / 2 g} \oplus \mathrm{F}_{3 / 2 g}, \\
& { }^{2} \mathrm{~T}_{2 g} \otimes \mathrm{E}_{1 / 2 g}=\mathrm{E}_{5 / 2 g} \oplus \mathrm{F}_{3 / 2 g} .
\end{aligned}
$$

We find all three half-valued terms from which the decays in $1 \mathrm{~s} 2 \mathrm{p}$ RIXS can occur.

The final state $\left(1 s^{2} 2 p^{5} 3 d^{6}\right)$ is again identical to the high spin case regardless of the crystal field effects, offering the three total symmetry IRREPs $\mathrm{E}_{1 / 2 u}, \mathrm{E}_{5 / 2 u}$, andF $\mathrm{F}_{3 / 2 u}$. As given in (33), (34), and (35), the decay paths are formally the same as in the high spin case reaching all three odd symmetry terms. The differences in the spectra therefore 


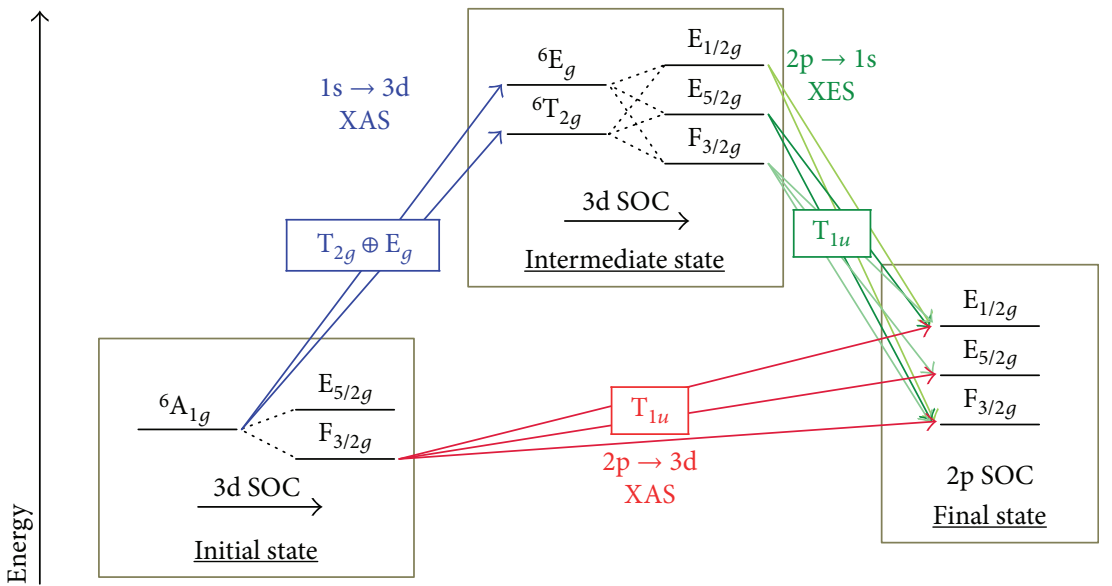

FIGURE 39: Multielectronic term scheme for $\mathrm{Fe}^{3+}\left(3 \mathrm{~d}^{5}\right)$ high spin comparing the1s $2 \mathrm{p}$ RIXS and direct $2 \mathrm{p}$ XAS pathways. Where SOC is included $(\mathcal{S}=5 / 2)$, the spin multiplicity is omitted.

relate to the difference of the intermediate state population via the first 1s XAS step. Again, the intermediate state plays the decisive role.

For $2 p$ XAS, starting from the $\mathrm{E}_{5 / 2 g}$ ground state, only two final state terms are reachable:

$$
\underbrace{\mathrm{E}_{5 / 2 g}}_{\text {GS }} \frac{\otimes \mathrm{T}_{1 u}}{2 \mathrm{pXAS}} \underbrace{\mathrm{E}_{1 / 2 u} \oplus \mathrm{F}_{3 / 2 u}}_{\text {Accessible FS terms }} .
$$

The results of the selection rules as discussed above are summarised in the combined term scheme shown in Figure 42.

The calculated 1s2p RIXS map for a low spin case (10D $q=3.9 \mathrm{eV})$ is given in Figure 43 .

In the left RIXS map in Figure 43(a), it appears that the quadrupole IRREP $\mathrm{T}_{2 g}$ enables to reach only the first fully symmetric ${ }^{2} \mathrm{~A}_{1 g}$ intermediate state symmetry. This term corresponds in the single electron picture to a $\left|t_{2 g}^{6} e_{g}^{0}\right\rangle$ configuration of the $3 \mathrm{~d}$ shell where all the $t_{2 g}$ orbitals occupied. The spin doublet emerges solely from the core hole in the $1 \mathrm{~s}$ shell.

The $2 p$ XES decay reaches two final states corresponding to the $t_{2 g}$ orbital full and a hole in the $2 \mathrm{p}$ shell, relating to one in the $\mathrm{L}_{3}$ and one in the $\mathrm{L}_{2}$. In the multielectronic picture, these two peaks correspond to the ${ }^{2} \mathrm{~A}_{1 g} \otimes{ }^{2} \mathrm{P}$ final states (see Figures 43 and 44). It is noteworthy that this peak is not reached in the $\mathrm{L}_{2}$-edge via the $2 \mathrm{p}$ XAS. This can be explained in the single electron picture of the initial state, which contains only one hole in the $t_{2 g}$ orbitals. The $\mathrm{L}_{2}{ }^{-}$ edge does not get a peak because it would require a spin-flip to fill the $t_{2 g}$ hole with an electron from the $2 p$ shell. In $1 \mathrm{~s} 2 \mathrm{p}$ RIXS, these two peaks arise only from the decay of the $2 p$ shell electron into the 1s shell, which is possible for both $\mathrm{L}_{3}$ and $\mathrm{L}_{2}$. The higher energy range of the final states is reached via the intermediate state probed by the quadrupole $\mathrm{E}_{g}$ IRREP (1s XAS operator).
5.5.3. $1 s 2 p$ RIXS Experiments of $3 d^{5}$ Systems. Examples of $3 d^{5}$ systems include $\mathrm{Mn}^{2+}$ and $\mathrm{Fe}^{3+}$. Experimental 1s2p RIXS spectra of $\mathrm{MnO}$ have been published by Kas et al. [39]. Compared with the calculations in Figure 40, the intensity ratio of the $\mathrm{T}_{2 g}$ and the $\mathrm{E}_{g}$ peak is inverted in the experiment, with the $\mathrm{E}_{g}$ peak at $[6540,638] \mathrm{eV}$ having the largest intensity. The same observation can be made for the $\mathrm{Fe}^{3+}$ systems $\mathrm{Fe}_{2} \mathrm{O}_{3}$ and iron impurities in $\mathrm{MgO}$ [14]. The tetrahedral $\mathrm{Fe}^{3+}$ contains a single 1s2p RIXS feature, reproduced by the calculations. 1s2p RIXS of a series of iron minerals has been published by Vercamer in his PhD thesis [44]. The 1s2p RIXS spectrum of octahedral low spin compounds $\mathrm{Fe}(\mathrm{III})(\mathrm{tacn})$ and $\mathrm{Fe}(\mathrm{III})$ cyanide has been measured and reproduced in multiplet calculations by Lundberg et al. [45].

5.6. $3 d^{6}$ Ground State System, for Example, $\mathrm{Fe}^{2+}$. In this part, we present the elementary differences between 1s XAS and 1s2p RIXS for the case of $\mathrm{Fe}^{2+}\left(3 \mathrm{~d}^{6} ; \mathrm{Co}^{3+}\right.$ analogue). As in the previous case(s), first, we gather the relevant informations which are summarised in a combining term scheme illustrating the transition paths in 1s2p RIXS (1s XAS, 2p $\mathrm{XES}$ ) and 1s XAS. The electronic configurations involved are summarised below:

$$
\begin{array}{r}
\text { 1s2p RIXS : } 1 \mathrm{~s}^{2} 2 \mathrm{p}^{6} 3 \mathrm{~d}^{6} \frac{\Delta \mathscr{J}= \pm 2}{1 \mathrm{XAS}} 1 \mathrm{~s}^{1} 2 \mathrm{p}^{6} 3 \mathrm{~d}^{7} \frac{\Delta \mathscr{J}=0, \pm 1}{2 \mathrm{pXES}} \\
\text { 2p XAS : } 1 \mathrm{~s}^{2} 2 \mathrm{p}^{6} 3 \mathrm{~s}^{6} 2 \mathrm{p}^{5} 3 \mathrm{~d}^{7}, \\
\stackrel{\Delta \mathscr{J}=0, \pm 1}{2 \mathrm{pXAS}} 1 \mathrm{~s}^{2} 2 \mathrm{p}^{5} 3 \mathrm{~d}^{7} .
\end{array}
$$

As in the previous case, the crystal field splitting energy $(10 \mathrm{D} q)$ and the pairing energy are in competition in a $3 \mathrm{~d}^{6}$ configuration. Thus, for sufficiently high values of $10 \mathrm{D} q$, the ground state changes from high spin ${ }^{5} \mathrm{~T}_{2 g}$ for $\left|t_{2 g}^{4} e_{g}^{2}\right\rangle$ to low spin ${ }^{1} \mathrm{~A}_{1 g}$ for $\left|t_{2 g}^{6} e_{g}^{0}\right\rangle$.

5.6.1. $\mathrm{Fe}^{2+}\left(3 d^{6}\right)$ in High Spin (Quintet Ground State). The initial state electronic configuration is $3 \mathrm{~d}^{6}$ (210 microstates) with the atomic ground state term ${ }^{5} \mathrm{D}$ (25 microstates). In 

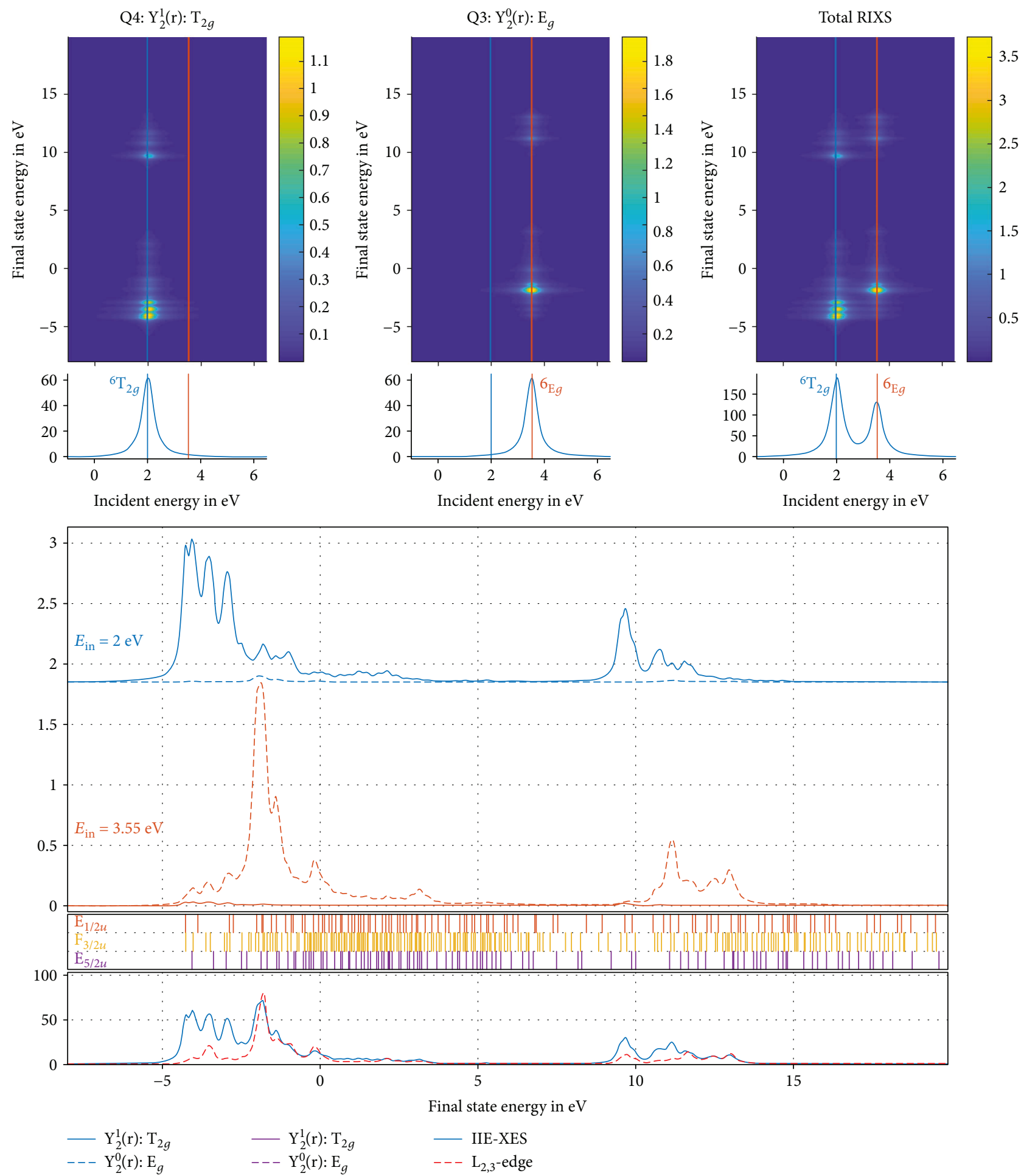

FIgUre 40: Calculated 1s2p RIXS spectrum for $\mathrm{Fe}^{3+}\left(3 \mathrm{~d}^{5}\right)$ for $10 \mathrm{D} q=1.5 \mathrm{eV}$ (high spin).

an $\mathrm{O}_{h}$ crystal field, the ${ }^{5} \mathrm{D}$ term splits into ${ }^{5} \mathrm{~T}_{2 g}$ for $\left|t_{2 g}^{4} e_{g}^{2}\right\rangle$ and ${ }^{5} \mathrm{E}_{g}$ for $\left|t_{2 g}^{3} e_{g}^{3}\right\rangle$. The inclusion of spin-orbit interaction $\left(\mathcal{S}=2 \rightarrow \otimes\left(\mathrm{E}_{g} \oplus \mathrm{T}_{2 g}\right)\right)$ yields the following splittings:

$$
{ }^{5} \mathrm{~T}_{2 g} \otimes\left(\mathrm{E}_{g} \oplus \mathrm{T}_{2 g}\right)=\mathrm{A}_{1 g} \oplus \mathrm{E}_{g} \oplus 2 \mathrm{~T}_{1 g} \oplus 2 \mathrm{~T}_{2 g} .
$$

Here, the term $\mathrm{T}_{2 g}$ lies the lowest in energy; thus, the ground state is ${ }^{5} \mathrm{~T}_{2 g}\left[\mathrm{~T}_{2 g}\right]\left(\mathrm{O}_{h}\right)$.

The intermediate state electron configuration $1 s^{1} 3 d^{7} 3 d^{7}$ $(2 \cdot 120=240$ microstates $)$ yields the atomic terms ${ }^{1,3,5} \mathrm{~F}$, ${ }^{1,3,5} \mathrm{P},{ }^{1,3} \mathrm{D},{ }^{1,3} \mathrm{G}$, and ${ }^{1,3} \mathrm{H}$ (Table 1). Using Table 2, one finds the branchings of the atomic states in an $\mathrm{O}_{h}$ crystal field which are analogue to the Tanabe-Sugano diagram of $3 \mathrm{~d}^{7}$. 


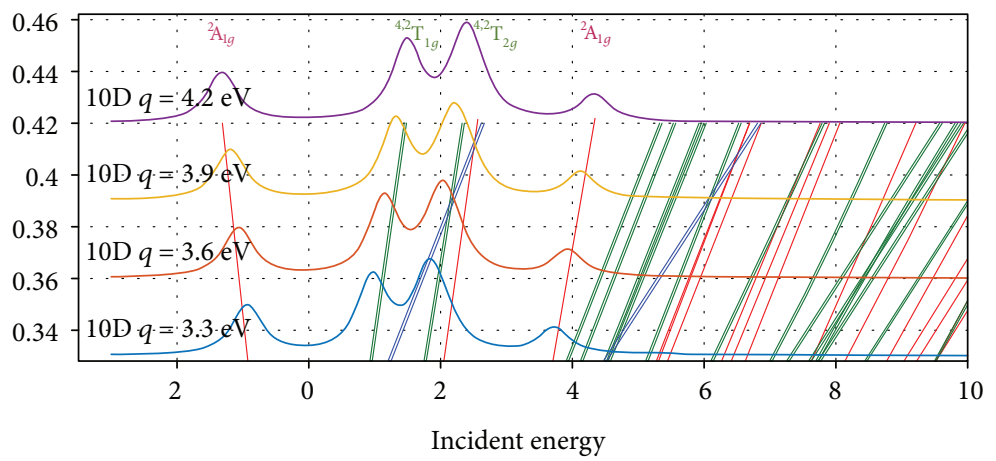

Figure 41: Low spin 1s XAS projections $(10 \mathrm{D} q=3.3 \mathrm{eV}$ to $4.2 \mathrm{eV})$ for $\mathrm{Fe}^{3+}\left(3 \mathrm{~d}^{5}\right)$ with ELD. In the single electron picture the $3 \mathrm{~d}$ shell has either $\left|t_{2 g}^{6} e_{g}^{0}\right\rangle$ for ${ }^{2} \mathrm{~A}_{1 g}$ or $\left|t_{2 g}^{5} e_{g}^{1}\right\rangle$ for ${ }^{4} \mathrm{~T}_{1 g}$ and ${ }^{4} \mathrm{~T}_{2 g}$, respectively.

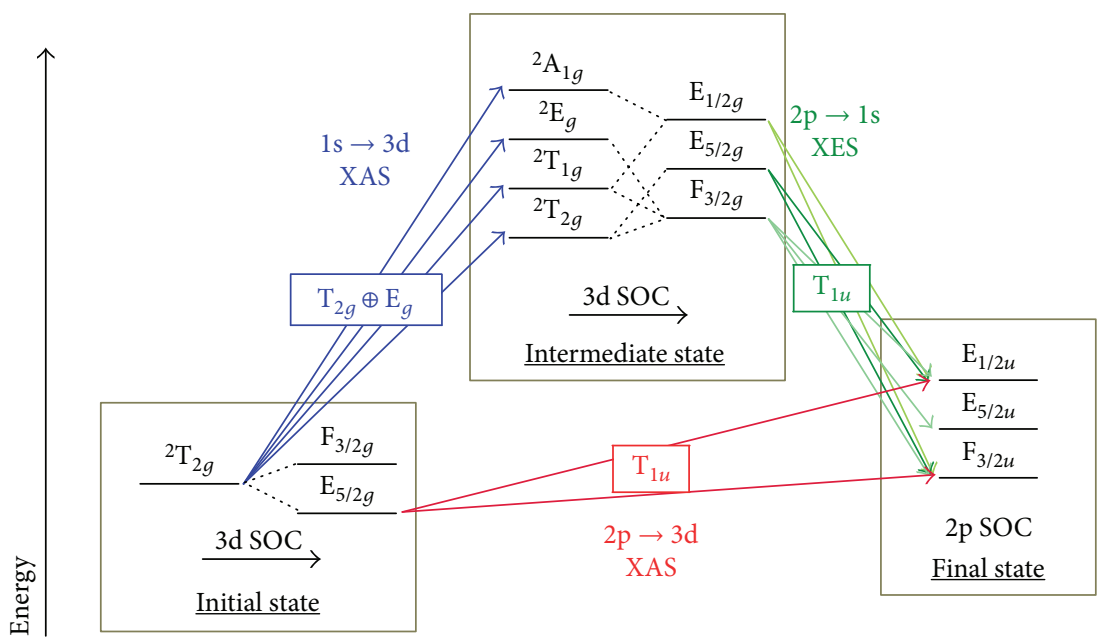

FIgURE 42: Multielectronic term scheme for $\mathrm{Fe}^{3+}\left(3 \mathrm{~d}^{5}\right)$ low spin comparing the $1 \mathrm{~s} 2 \mathrm{p}$ RIXS and direct $2 \mathrm{p}$ XAS pathways. Where SOC is considered, the spin multiplicity was omitted.

Because the 1s XAS is spin-conserving, only quintet intermediate states are reached in the absorption, corresponding to the crystal field terms derived from ${ }^{5} \mathrm{~F}$ and ${ }^{5} \mathrm{P}:{ }^{5} \mathrm{~A}_{2 g},{ }^{5} \mathrm{~T}_{2 g}$, ${ }^{5} \mathrm{~T}_{1 g}$, and ${ }^{5} \mathrm{~T}_{1 g}$, respectively. For $1 \mathrm{~s}$ XAS, starting from the ${ }^{5} \mathrm{~T}_{2 g}$ ground state term, the reachable high spin intermediate state terms are

$$
\begin{aligned}
& { }^{5} \mathrm{~T}_{2 g} \stackrel{\otimes \mathrm{T}_{2 g}}{\longrightarrow}{ }^{5} \mathrm{~A}_{1 g} \oplus^{5} \mathrm{E}_{g} \oplus^{5} \mathrm{~T}_{1 g} \oplus^{5} \mathrm{~T}_{2 g}, \\
& \underbrace{{ }^{5} \mathrm{~T}_{2 g}}_{\text {GS }} \stackrel{{ }^{\otimes \mathrm{E}_{g}}}{\text { 1s XAS }} \underbrace{{ }^{5} \mathrm{~T}_{1 g} \oplus^{5} \mathrm{~T}_{2 g}}_{\text {Reachable IS (high spin) }} .
\end{aligned}
$$

The reachable intermediate state terms of $\mathrm{Fe}^{2+}\left(3 \mathrm{~d}^{6}\right)$ in high spin are therefore ${ }^{5} \mathrm{~T}_{1 g}$ and ${ }^{5} \mathrm{~T}_{2 g}$. In other words, though the ${ }^{5} \mathrm{~A}_{1 g}$ and ${ }^{5} \mathrm{E}_{g}$ symmetries are formally reachable in $1 \mathrm{~s}$ XAS, the intermediate state does not offer this IRREP to be populated. Furthermore, though an ${ }^{5} \mathrm{~A}_{2 g}$ IRREP exists, the quadrupole selection rules do not allow it to be reachable in 1s XAS (see Figure 45). However, the small 3d SOC may induce some mixing enabling also weak transitions into this IRREP.

Adding SOC $\left(\mathcal{S}=2 \rightarrow \otimes\left(\mathrm{E}_{g} \oplus \mathrm{T}_{2 g}\right)\right)$ to the reachable IS terms leads to the splittings in the IS:

$$
\begin{aligned}
{ }^{5} \mathrm{~A}_{1 g} \otimes\left(\mathrm{E} \oplus \mathrm{T}_{2}\right) & =\mathrm{E} \oplus \mathrm{T}_{2}, \\
{ }^{5} \mathrm{~T}_{1 g} \otimes\left(\mathrm{E}_{g} \oplus \mathrm{T}_{2 g}\right) & =\left(\mathrm{T}_{1 g} \oplus \mathrm{T}_{2 g}\right) \oplus\left(\mathrm{A}_{2 g} \oplus \mathrm{E}_{g} \oplus \mathrm{T}_{1 g} \oplus \mathrm{T}_{2 g}\right), \\
{ }^{5} \mathrm{~T}_{2 g} \otimes\left(\mathrm{E}_{g} \oplus \mathrm{T}_{2 g}\right) & =\left(\mathrm{T}_{1 g} \oplus \mathrm{T}_{2 g}\right) \oplus\left(\mathrm{A}_{1 g} \oplus \mathrm{E}_{g} \oplus \mathrm{T}_{1 g} \oplus \mathrm{T}_{2 g}\right) .
\end{aligned}
$$

We find the decays in 1s2p RIXS can occur from all integer symmetries $\left(\mathrm{A}_{1 g}, \mathrm{~A}_{2 g}, \mathrm{E}_{g}, \mathrm{~T}_{1 g}\right.$, and $\left.\mathrm{T}_{2 g}\right)$.

The final state electron configuration $1 \mathrm{~s}^{2} 2 \mathrm{p}^{5} 3 \mathrm{~d}^{7}$ contains $6 \cdot 120=720$ microstates due to the hole in the $2 \mathrm{p}$ shell spanning $\mathscr{J}$ from 0 to 7 . In an $\mathrm{O}_{h}$ crystal field, these terms branch into the five integer total symmetry IRREPs $\mathrm{A}_{1 g}, \mathrm{~A}_{2 g}, \mathrm{E}_{g}, \mathrm{~T}_{1 g}$, andT $_{2 g}$. The2p XES decays can occur from any of the populated intermediate state terms; thus, the accessible final state terms are again derived with the dipole $\mathrm{T}_{1 u}$ IRREP as given in (28), (29), (30), (31), and (32). In short, all "ungerade" integer IRREPs are reachable in the final state of $2 \mathrm{p}$ XES. 

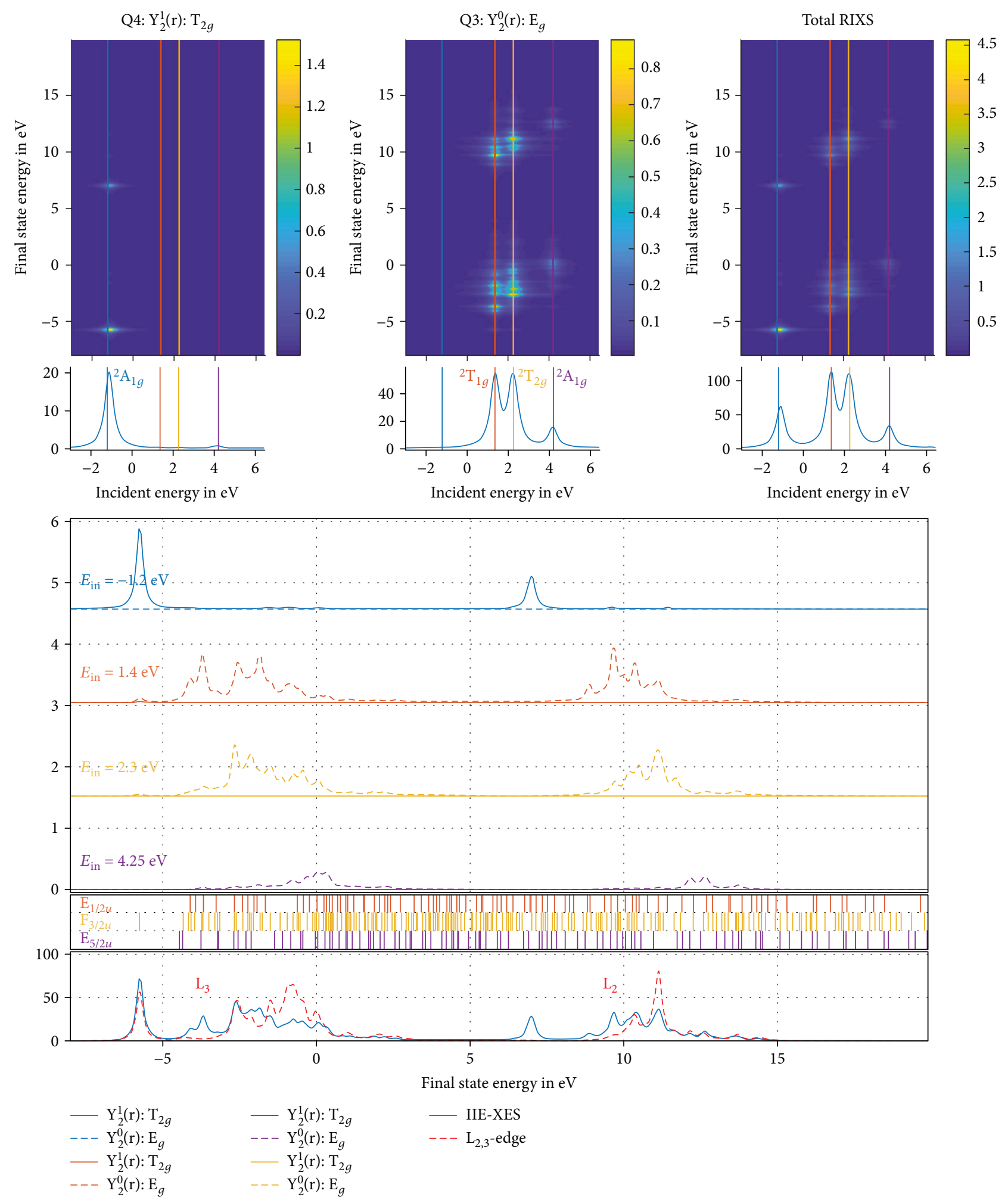

FIGURE 43: Calculated 1s2p RIXS spectrum for $\mathrm{Fe}^{3+}\left(3 \mathrm{~d}^{5}\right)$ for $10 \mathrm{D} q=3.9 \mathrm{eV}$ (low spin). 


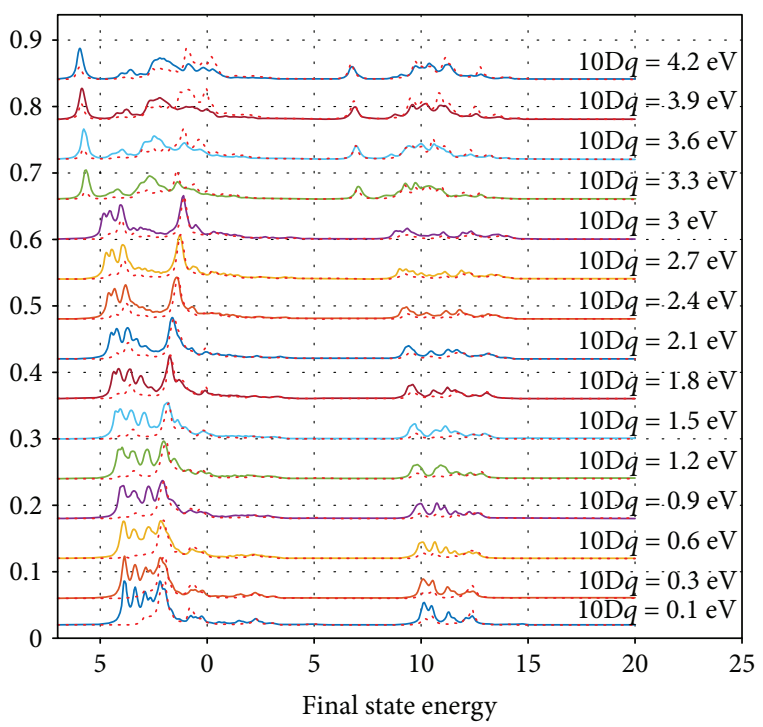

FIGURE 44: $2 \mathrm{p}$ XES projections for $\mathrm{Fe}^{3+}\left(3 \mathrm{~d}^{5}\right)$ for $10 \mathrm{D} q=0.1 \mathrm{eV}$ to $4.2 \mathrm{eV}$. The high spin to low spin transition between $10 \mathrm{D} q \approx 3.0 \mathrm{e}$ $\mathrm{V}$ and $3.3 \mathrm{eV}$ also induces visible changes in the $2 \mathrm{p}$ XES. The most notable is the appearance of the two peaks related to the first ${ }^{2} \mathrm{~A}_{1 \mathrm{~g}}$ intermediate state symmetry around $E_{T}<-5 \mathrm{eV}$ and $E_{T} \approx 7 \mathrm{eV}$.

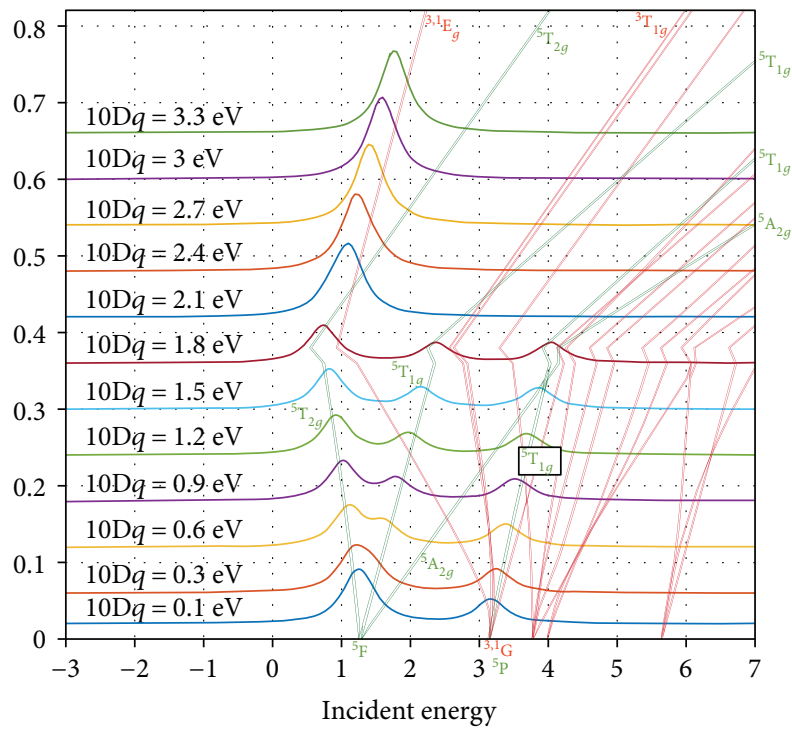

FIGURE 45: 1s XASprojections of the calculated 1s2p RIXSspectrum forFe $e^{2+}\left(3 \mathrm{~d}^{6}\right)$ showing the high spin to low spin transition just above $10 \mathrm{D} q \approx 1.8 \mathrm{eV}$.

In $2 \mathrm{p}$ XAS, starting from the $\mathrm{T}_{2 g}$ total symmetry IRREP term and using the $\mathrm{T}_{1 u}$ IRREP, the reachable final states are again identical to the $2 \mathrm{p}$ XES final state term reachable from the $\mathrm{T}_{2 g}$ intermediate state IRREP as given (32).

Altogether, this enables to draw the term scheme for $\mathrm{Fe}^{2+}$ $\left(3 d^{6}\right)$ in high spin as shown in Figure 46.

The result of the calculation for high spin $\mathrm{Fe}^{2+}\left(3 \mathrm{~d}^{6}\right)$ for $10 \mathrm{D} q=0.9 \mathrm{eV}$ is shown in Figure 47.

We note that the quadrupole $\mathrm{T}_{2 g}$ IRREP (left RIXS map in Figure 47(a)) is dominated by transitions into the ${ }^{5} \mathrm{~T}_{2 g}$ intermediate state symmetry, while the quadrupole $\mathrm{E}_{g}$ IRREP (middle RIXS map in Figure 47(a)) leads mostly into the $\mathrm{T}_{1 \mathrm{~g}}$ intermediate state IRREPs. As predicted above, the 1s XAS spectra gain their intensity only from two intermediate state symmetries, the ${ }^{5} \mathrm{~T}_{1 g}$ and ${ }^{5} \mathrm{~T}_{2 g}$ IRREPs. The ${ }^{5} \mathrm{~A}_{2 g}$ intermediate state symmetry does not contribute to the spectrum as can be seen in Figure 45.

5.6.2. $\mathrm{Fe}^{2+}\left(3 d^{6}\right)$ in Low Spin (Singlet Ground State). For a sufficiently large crystal field splitting $(10 \mathrm{Dq})$, a $3 \mathrm{~d}^{6}$ electronic configuration results in a low spin configuration with all $t_{2 g}$ orbitals fully occupied $\left(\left|t_{2 g}^{6} e_{g}^{0}\right\rangle\right)$. This corresponds to the totally symmetric ground state term ${ }^{1} \mathrm{~A}_{1 g}$ (1 microstate) derived from the atomic term ${ }^{1} \mathrm{I}$ (Table 1). The inclusion of spin-orbit interaction $\left(\mathcal{S}=0 \rightarrow \otimes \mathrm{A}_{1 g}\right)$ gives the total ground state as ${ }^{1} \mathrm{~A}_{1 g}\left[\mathrm{~A}_{1 g}\right]\left(\mathrm{O}_{h}\right)$.

The intermediate state electron configuration $1 \mathrm{~s}^{1} 3 \mathrm{~d}^{7}$ is still the same as in the high spin case. However, for high crystal fields $(10 \mathrm{D} q)$, the order of the intermediate state energy levels is different in low spin. In the low spin case, the initial state being a spin singlet, the spin-conserving 1s XAS will only reach spin singlet intermediate states (Table 3):

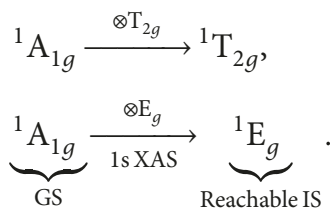

Although there are ${ }^{1} \mathrm{~T}_{2 g}$ and ${ }^{1} \mathrm{E}_{g}$ IRREPs existing in the intermediate state, only the ${ }^{1} \mathrm{E}_{g}$ derived from the ${ }^{1} \mathrm{G}$ atomic term can be reached. This can be understood from the $\Delta \mathscr{L}= \pm 2$ quadrupole selection rule, which allows only to reach the ${ }^{1} \mathrm{G}$ atomic term $(\mathscr{L}=4)$ from the ${ }^{1}$ I atomic ground state term $(\mathscr{L}=6)$. The quadrupole 1 s XAS formally can, according to the selection rules, only reach the ${ }^{1} \mathrm{~T}_{2 g}$ and ${ }^{1} \mathrm{E}_{g}$ IRREPs from this term. But finally, the transition to the ${ }^{1} \mathrm{~T}_{2 g}$ intermediate state symmetry would imply a two-electron transition from $\left|t_{2 g}^{6} e_{g}^{0}\right\rangle$ to $\left|t_{2 g}^{5} e_{g}^{2}\right\rangle$, which has a very low probability. Hence, only the ${ }^{1} \mathrm{E}_{g}$ IRREP from the ${ }^{1} \mathrm{G}$ atomic term is populated thus adding intensity to the $1 \mathrm{~s}$ XAS spectrum as visible in Figure 48.

For the $2 \mathrm{p}$ XES decays in 1s2p RIXS, spin-orbit interaction in the intermediate state must be considered $(\mathcal{S}=0 \rightarrow$ $\otimes \mathrm{A}_{1 g}$ ) leading the identities:

$$
\underbrace{{ }^{1} \mathrm{E}_{g}}_{\text {reachable IS }} \otimes \mathrm{A}_{1 g}=\mathrm{E}_{g} .
$$

The final state electron configuration $1 s^{2} 2 \mathrm{p}^{5} 3 \mathrm{~d}^{7}$ is again identical to the high spin case regardless of the crystal field effects, offering the five total symmetry IRREPs $\mathrm{A}_{1 u}, \mathrm{~A}_{2 u}, \mathrm{E}_{u}, \mathrm{~T}_{1 u}$, and $\mathrm{T}_{2 u}$. The $2 \mathrm{p}$ XES decaying from the populated intermediate state term $\mathrm{E}_{g}$ can reach with the dipole IRREP $\mathrm{T}_{1 u}$ the final state terms $\mathrm{T}_{1 u}$ and $\mathrm{T}_{2 u}$ as given in (30). 


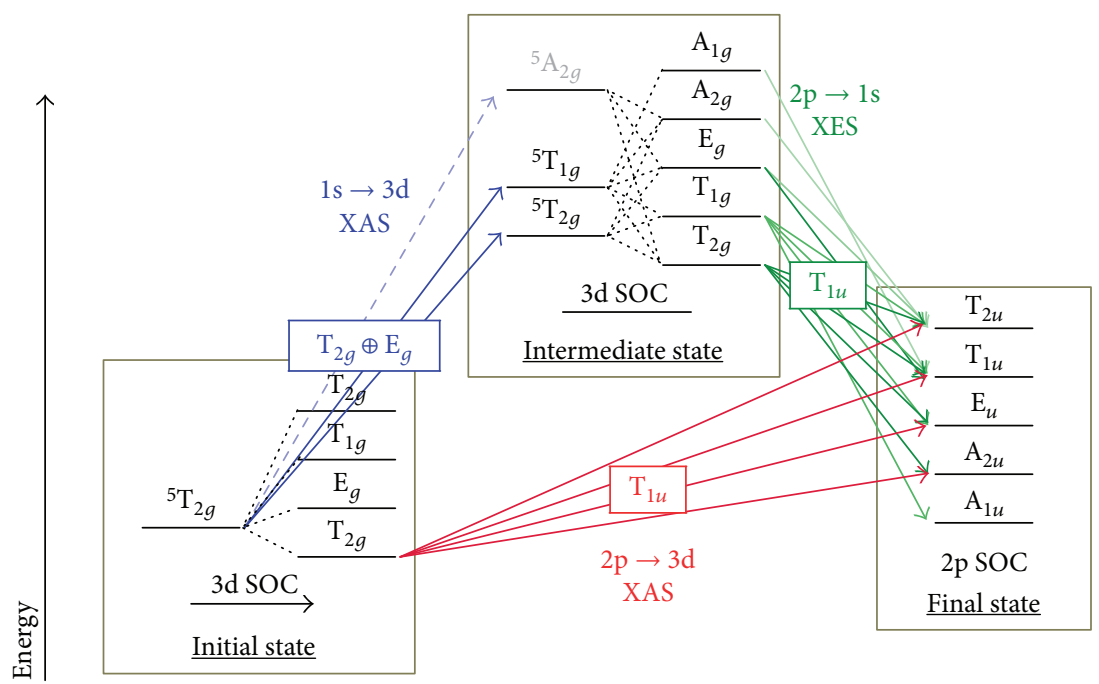

FIgURE 46: Multielectronic term scheme for $\mathrm{Fe}^{2+}\left(3 \mathrm{~d}^{6}\right)$ (high spin) comparing the $1 \mathrm{~s} 2 \mathrm{p}$ RIXS and $2 \mathrm{p}$ XAS pathways. Where SOC is considered, the spin multiplicity is omitted as its meaning is lost.

On the contrary, the $2 \mathrm{p}$ XAS, starting from the total symmetric $\mathrm{A}_{1 g}$ ground state symmetry, can reach only the $\mathrm{T}_{1 u}$ final state IRREP.

This again enables altogether to draw the combined term scheme comparing the two-step 1s2p RIXS with the direct dipole 2p XAS pathways in Figure 49.

The resulting calculated 1s $2 p$ RIXS for $\mathrm{Fe}^{2+}\left(3 \mathrm{~d}^{6}\right)$ is shown in Figure 50. As already discussed, the 1s2p RIXS is dominated by the $\mathrm{E}_{g}$ intermediate state symmetry. As can be seen, the quadrupole $\mathrm{T}_{2 g}$ IRREP has overall a negligible contribution to the total RIXS. From the term scheme in Figure 49 , one can furthermore derive that the difference between the integrated $2 p$ XES (IIE) and the direct $2 p$ XAS emerges from the $\mathrm{T}_{2 u}$ final state IRREP which can only be reached in 1 s $2 p$ RIXS.

5.6.3. $1 s 2 p$ RIXS Experiments of $3 \mathrm{~d}^{6}$ Systems. $3 \mathrm{~d}^{6}$ systems include $\mathrm{Fe}^{2+}$ and $\mathrm{Co}^{3+}$. A large number of experimental $1 \mathrm{~s} 2 \mathrm{p}$ RIXS spectra of $\mathrm{Fe}^{2+}$ systems have been published. Vercamer measured a large series of $\mathrm{Fe}^{2+}$ minerals in his $\mathrm{PhD}$ thesis [44]. The tetrahedral and octahedral $\mathrm{Fe}^{2+}$ oxides/minerals have been studied and reproduced by multiplet calculations $[5,17,46]$. Guo et al. [47] studied low spin $\mathrm{Fe}^{2+}$ cyanide complexes. Lundberg et al. [45] compared these experiments to the tacn complex and reproduced the spectra from multiplet calculations. Leidel et al. [48] studied iron hydrogenase complexes. Vanko et al. [49] studied the time evolution of the 1s2p RIXS planes. The static spectra of the low spin systems $\left[\mathrm{Fe}(\text { terpy })_{2}\right]^{2+}$ and $\left[\mathrm{Fe}(\text { bipy })_{3}\right]^{2+}$ and the laser-excited spectrum of $\left[\mathrm{Fe}(\text { terpy })_{2}\right]^{2+}$ show a partial transition to a high spin ground state as is confirmed in the calculations.

In case of $\mathrm{Co}^{3+}$, all measured systems were low $\operatorname{spin}{ }^{1} \mathrm{~A}_{1 g}$. Vanko et al. [7] compared an isolated $\mathrm{Co}^{3+}$ complex with a series of cobalt oxides. They nicely show the presence of the nonlocal peaks as a function of the Co-O-Co angle.
5.7. $3 d^{7}$ Ground State System, for Example, $\mathrm{Co}^{2+}$. In this part, we present the elementary differences between $1 \mathrm{~s} 2 \mathrm{p}$ RIXS and $2 p$ XAS for the case of $\mathrm{Co}^{2+}\left(3 \mathrm{~d}^{7}\right)$. First, we gather the relevant information analogue to the previous cases which are again summarised in the combining term scheme in Figure 39 term scheme illustrating the transition paths involved (Figures 51 and 52). The electronic configurations involved are summarised below:

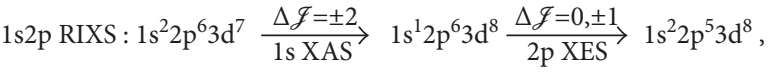

$$
\begin{aligned}
& \text { 2p XAS : } 1 \mathrm{~s}^{2} 2 \mathrm{p}^{6} 3 \mathrm{~d}^{7} \longrightarrow \frac{\Delta \mathscr{J}=0, \pm 1}{2 \mathrm{pXAS}} \longrightarrow 1 \mathrm{~s}^{2} 2 \mathrm{p}^{5} 3 \mathrm{~d}^{8} .
\end{aligned}
$$

Also, a $3 \mathrm{~d}^{7}$ system encounters, for sufficiently high values of $10 \mathrm{D} q$, a change of the ground state from ${ }^{4} \mathrm{~T}_{2 g}$ for $\left|t_{2 g}^{5} e_{g}^{2}\right\rangle$ (high spin) to ${ }^{2} \mathrm{E}_{g}$ for $\left|t_{2 g}^{6} e_{g}^{1}\right\rangle$ (low spin).

5.7.1. $\mathrm{Co}^{2+}\left(3 d^{7}\right)$ in High Spin (Quartet Ground State). For the initial state configuration $3 \mathrm{~d}^{7}$ (120 microstates), the high spin ground state term symbol is ${ }^{4} \mathrm{~F}$ (Table 1 ), which branches in $\mathrm{O}_{h}$ into ${ }^{4} \mathrm{~T}_{1 g},{ }^{4} \mathrm{~T}_{2 g}$, and ${ }^{4} \mathrm{~A}_{2 g}$, where the ${ }^{4} \mathrm{~T}_{1 g}$ term with 12 microstates is the lowest in energy. The inclusion in spinorbit interaction $\left(\mathcal{S}=3 / 2 \rightarrow \otimes \mathrm{F}_{3 / 2 g}\right)$ yields the subsequent splitting of ${ }^{4} \mathrm{~T}_{1 g}$ term:

$$
{ }^{4} \mathrm{~T}_{1 g} \otimes \mathrm{F}_{3 / 2 g}=\mathrm{E}_{1 / 2 g} \oplus \mathrm{E}_{5 / 2 g} \oplus 2 \mathrm{~F}_{3 / 2 g} .
$$

The ground state ${ }^{4} \mathrm{~T}_{1 g}$ splits due to $3 \mathrm{~d}$ SOC into the three odd symmetries $\mathrm{E}_{1 / 2 \mathrm{~g}}, \mathrm{E}_{5 / 2 \mathrm{~g}}$, and $\mathrm{F}_{3 / 2 \mathrm{~g}}$ from which the term $\mathrm{E}_{1 / 2 \mathrm{~g}}$ is lowest in energy; hence, the combined ground state is ${ }^{4} \mathrm{~T}_{1 g}\left[\mathrm{E}_{1 / 2 g}\right]\left(\mathrm{O}_{h}\right)$. 

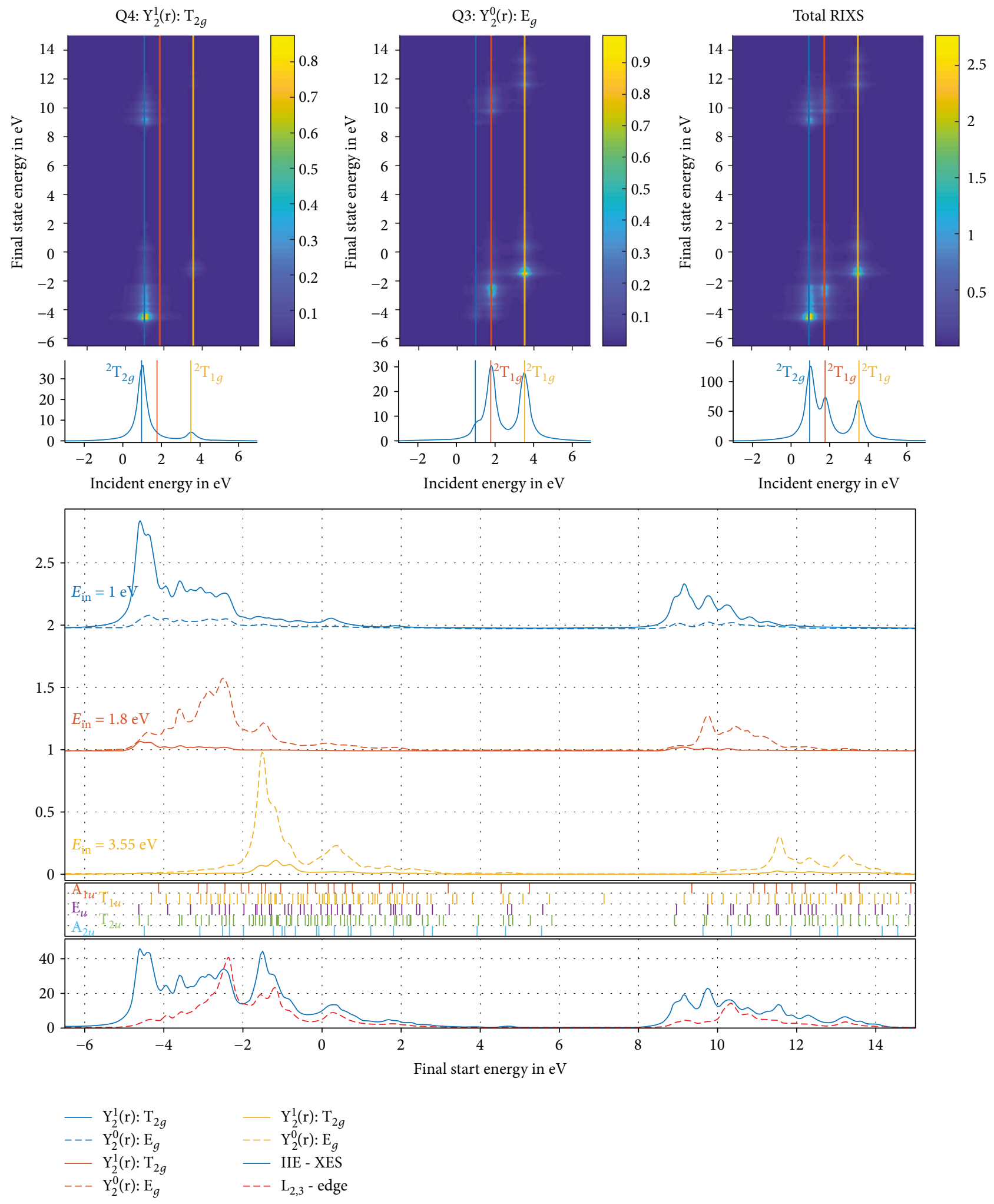

FIgURE 47: Calculated 1s2p RIXS spectra and slices for $\mathrm{Fe}^{2+}\left(3 \mathrm{~d}^{6}\right)$ for $10 \mathrm{D} q=0.9 \mathrm{eV}$ (high spin).

The intermediate state electron configuration is $1 \mathrm{~s}^{1} 3 \mathrm{~d}^{8}$ $(2 \cdot 45=90$ microstates $)$. The atomic terms are ${ }^{2,4} \mathrm{~F},{ }^{2,4} \mathrm{P},{ }^{2} \mathrm{~S}$, ${ }^{2} \mathrm{D}$, and ${ }^{2} \mathrm{G}$ (Table 1). Using Table 2, one finds the branchings of the atomic states in an $\mathrm{O}_{h}$ crystal field which are analogue to the Tanabe-Sugano diagram of $3 \mathrm{~d}^{8}$. The spin-conserving 1s XAS reaches only spin quartet intermediate states, which corresponds to the crystal field terms issued from ${ }^{4} \mathrm{~F}$ and ${ }^{4} \mathrm{P}:{ }^{4} \mathrm{~A}_{2 g},{ }^{4} \mathrm{~T}_{2 g},{ }^{4} \mathrm{~T}_{1 g}$, and ${ }^{4} \mathrm{~T}_{1 g}$, respectively. 


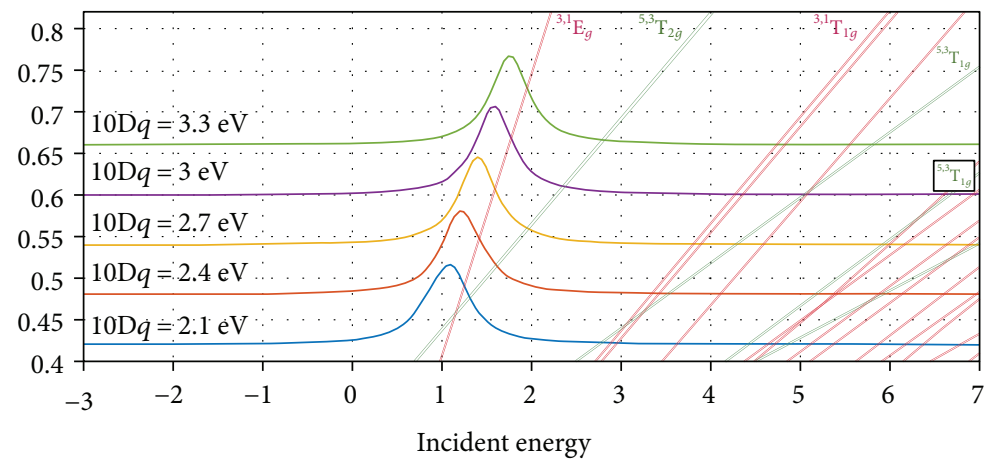

FIGURE 48: 1s XAS projections of the calculated $1 \mathrm{~s} 2 \mathrm{p}$ RIXS spectrum for $\mathrm{Fe}^{2+}\left(3 \mathrm{~d}^{6}\right)$ showing low spin part above $10 \mathrm{D} q \approx 1.8 \mathrm{eV}$ only. It reveals only the $\mathrm{E}_{g}$ symmetry is giving a significant contribution.

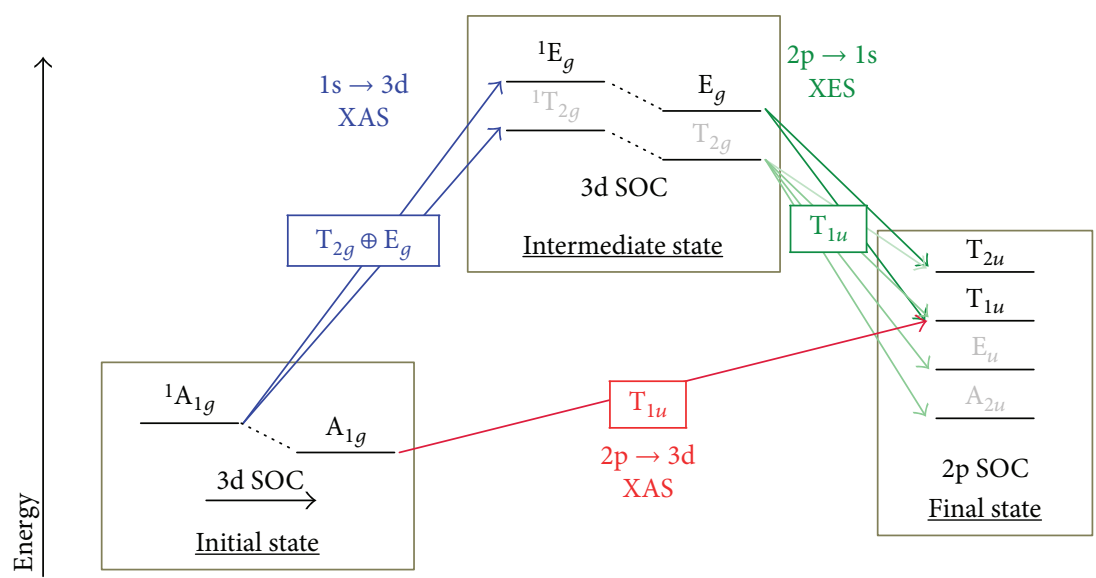

FIgURE 49: Multielectronic term scheme for $\mathrm{Fe}^{2+}\left(3 \mathrm{~d}^{6}\right)$ in low spin comparing the $1 \mathrm{~s} 2 \mathrm{p}$ RIXS and direct $2 \mathrm{p}$ XAS pathways. We note its similarity to the $\mathrm{Ti}^{4+}\left(3 \mathrm{~d}^{0}\right)$ case shown in Figure 15.

Starting from the high spin ground state term ${ }^{4} \mathrm{~T}_{1 g}$, the two quadrupole IRREPs give the reachable intermediate state terms:

$$
\begin{aligned}
& { }^{4} \mathrm{~T}_{1 g} \stackrel{\otimes \mathrm{T}_{2 g}}{\longrightarrow}{ }^{4} \mathrm{~A}_{2 g} \oplus{ }^{4} \mathrm{E}_{g} \oplus{ }^{4} \mathrm{~T}_{1 g} \oplus{ }^{4} \mathrm{~T}_{2 g},
\end{aligned}
$$

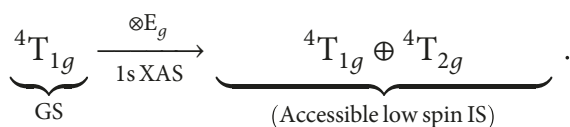

The reachable 1s XAS intermediate state terms in high spin are therefore ${ }^{4} \mathrm{~A}_{2 g},{ }^{4} \mathrm{~T}_{1 g}$, and ${ }^{4} \mathrm{~T}_{2 g}$. Here, it shall be noticed that an $\mathrm{E}_{g}$ symmetry would be reachable by the quadrupole selection rules, but the intermediate state does not offer any (quartet) ${ }^{4} \mathrm{E}_{g}$ symmetries. The 1 s XAS projections displayed in Figure 53 confirm these findings.

Applying the $3 \mathrm{~d}$ spin-orbit interaction $\left(\mathcal{S}=3 / 2 \rightarrow \otimes \mathrm{F}_{3 / 2 g}\right)$ to the three reachable terms yields the splittings in the intermediate state:

$$
\begin{aligned}
& { }^{4} \mathrm{~A}_{2 g} \otimes \mathrm{F}_{3 / 2 g}=\mathrm{F}_{3 / 2 g}, \\
& { }^{4} \mathrm{~T}_{1 g} \otimes \mathrm{F}_{3 / 2 g}=\mathrm{E}_{1 / 2 g} \oplus \mathrm{E}_{5 / 2 g} \oplus 2 \cdot \mathrm{F}_{3 / 2 g}, \\
& { }^{4} \mathrm{~T}_{2 g} \otimes \mathrm{F}_{3 / 2 g}=\mathrm{E}_{1 / 2 g} \oplus \mathrm{E}_{5 / 2 g} \oplus 2 \cdot \mathrm{F}_{3 / 2 g} .
\end{aligned}
$$

The result is that all three half-valued IRREPs appear more than once and hence the 2 p XES can decay from any of these terms.

The final state $\left(1 s^{2} 2 p^{5} 3 d^{8}\right)$ contains $\cdot 6 \cdot 45=270$ microstates due to the hole in the 2 p shell spanning $\mathscr{J}$ from $1 / 2$ to $11 / 2$. In an $\mathrm{O}_{h}$ crystal field, these terms branch into the three total symmetry IRREPs $\mathrm{E}_{1 / 2 u}, \mathrm{E}_{5 / 2 u}$, and $\mathrm{F}_{3 / 2 u}$. The $2 \mathrm{p}$ XES starts from either $\mathrm{E}_{1 / 2 g}, \mathrm{E}_{5 / 2 g}$, or $\mathrm{F}_{3 / 2 g}$, and we find ((33), (34), and (35)) that all three odd terms are also reachable in the 1s $2 \mathrm{p}$ RIXS final state.

The $2 \mathrm{p}$ XAS on the other hand, starting from the $\mathrm{E}_{1 / 2 \mathrm{~g}}$ ground state term, can only reach the $\mathrm{E}_{1 / 2 u}$ and $\mathrm{F}_{3 / 2 u}$ final state terms (Table 4).

A summary of the findings above is shown below as a combined term scheme in Figure 51.

We conclude this part with the corresponding calculations of the $1 \mathrm{~s} 2 \mathrm{p}$ RIXS for $\mathrm{Co}^{2+}\left(3 \mathrm{~d}^{7}\right)$ for a crystal field energy of $10 \mathrm{Dq}=0.9 \mathrm{eV}$ which is shown in Figure 54 .

It can be noted that the RIXS map for the quadrupole $\mathrm{T}_{2 g}$ IRREP (Figure 54(a), left) is dominated by the 1s XAS transitions into the ${ }^{4} \mathrm{~A}_{2 g}$ intermediate state symmetry which corresponds in the single electron picture to a $\left|t_{2 g}^{6} e_{g}^{2}\right\rangle$ configuration. However, as given in expression (75), it has also contributions into the two $\mathrm{T}_{1 g}$ intermediate state symmetries 

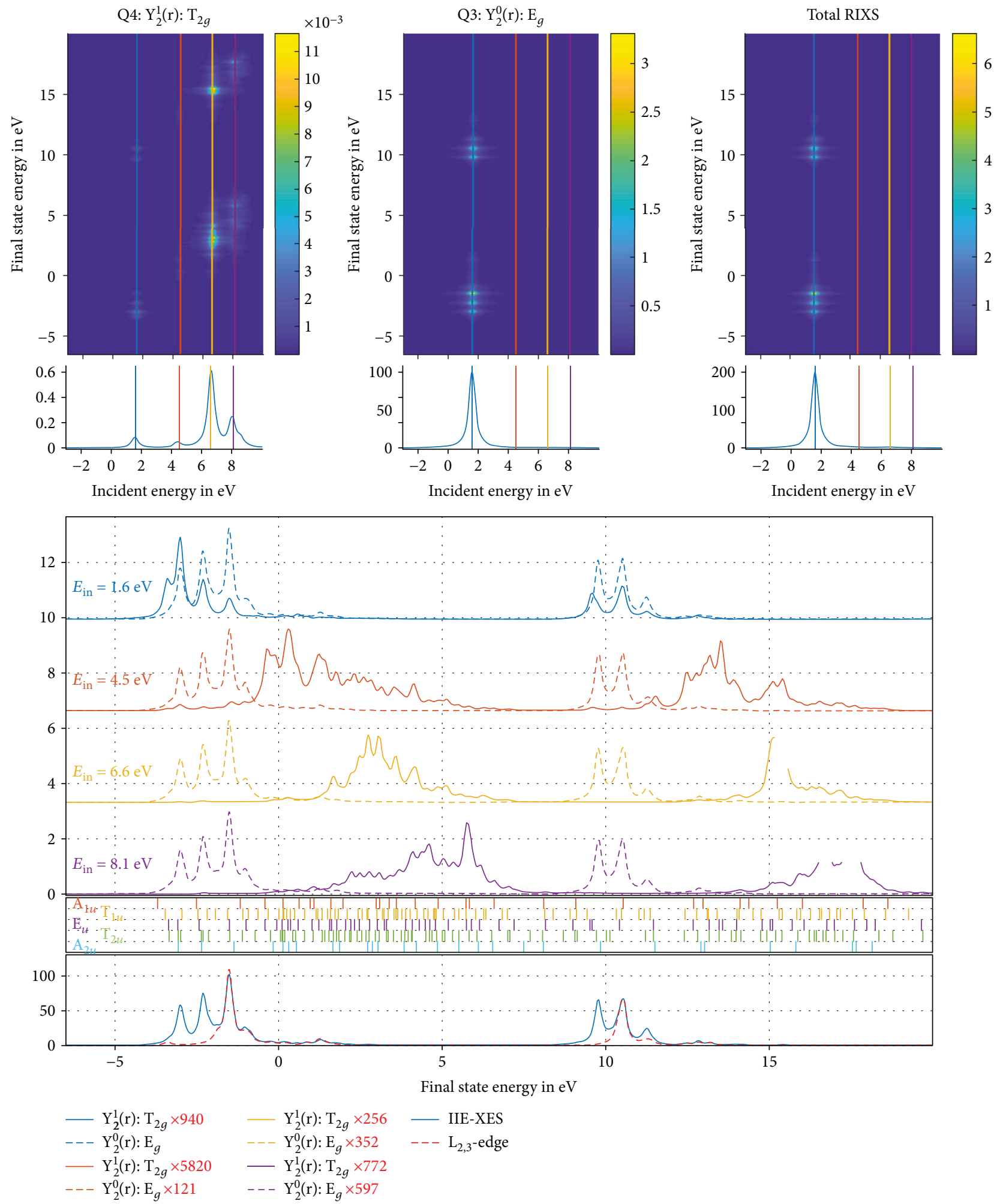

Figure 50: Calculated 1s2p RIXS spectrum for $\mathrm{Fe}^{2+}\left(3 \mathrm{~d}^{6}\right)$ for $10 \mathrm{D} q=3.0 \mathrm{eV}$ (low spin).

though they are weak with respect to the $\mathrm{A}_{2 g}$ contribution. The stick spectrum here is not as dense as in some of the cases discussed above, and this enables to identify also some of the reachable final state terms. For example, the strong peak at $E_{T} \approx-4.5 \mathrm{eV}$ relating to the decay from the ${ }^{4} \mathrm{~A}_{2 g}$ intermediate state symmetry corresponds to the two final state terms $\mathrm{E}_{1 / 2 u}$ and $\mathrm{F}_{3 / 2 u}$. This can be understood considering that the ${ }^{4} \mathrm{~A}_{2 g}$ branches due to SOC into a $\mathrm{F}_{3 / 2 g}$ intermediate state symmetry, which can reach the above two final state terms via the dipole $\mathrm{T}_{1 u}$ IRREP. 


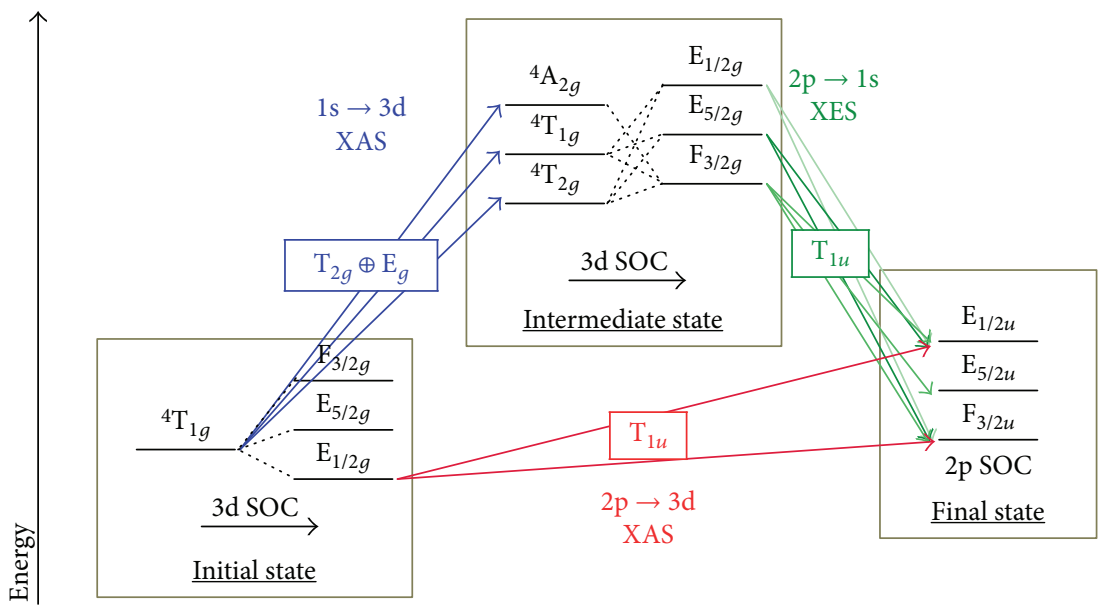

FIGURE 51: Multielectronic term scheme for $\mathrm{Co}^{2+}\left(3 \mathrm{~d}^{7}\right)$ in high spin comparing the 1s2p RIXS and direct 2p XAS pathways. Also, the spin multiplicity was omitted where the spin-orbit interaction is included.

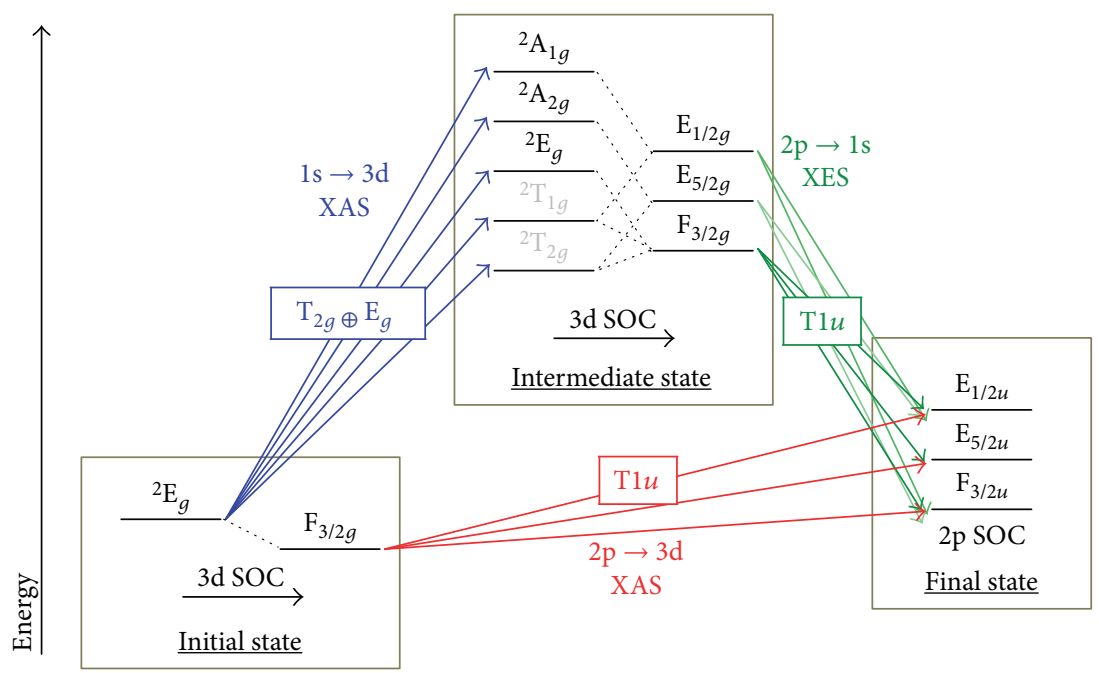

FIGURE 52: Multielectronic term scheme for a $\mathrm{Co}^{2+}$ ion $\left(3 \mathrm{~d}^{7}\right)$ in low spin comparing the $1 \mathrm{~s} 2 \mathrm{p}$ RIXS and direct $2 \mathrm{p}$ XAS pathways. Where SOC is included, the spin multiplicity is omitted.

The quadrupole IRREP $\mathrm{E}_{g}$ on the other hand shows only contributions into the ${ }^{4} \mathrm{~T}_{1 g}$ and ${ }^{4} \mathrm{~T}_{2 g}$ intermediate state symmetries as predicted in expression (76).

And finally, it should be noted that the 1s XAS projections in Figure 53 show with increasing crystal field energy $10 \mathrm{D} q$ the branching of the ${ }^{4} \mathrm{~F}$ into the three IRREPs ${ }^{4} \mathrm{~A}_{2}$, ${ }^{4} \mathrm{~T}_{2 g}$, and ${ }^{4} \mathrm{~T}_{1 g}$, while the atomic term ${ }^{4} \mathrm{P}$ translates in an $\mathrm{O}_{h}$ crystal field only into a ${ }^{4} \mathrm{~T}_{1 g}$ symmetry.

5.7.2. $\mathrm{Co}^{2+}\left(3 d^{7}\right)$ in Low Spin (Doublet Ground State). At high crystal field energies, the $3 \mathrm{~d}^{7}$ initial state gives the low spin ground state term ${ }^{2} \mathrm{E}_{g}$ (4 microstates) arising from the atomic term ${ }^{2} \mathrm{~F}$ which corresponds to the electronic configuration $\left|t_{2 g}^{6} e_{g}^{1}\right\rangle$. The inclusion of spin-orbit interaction $\left(\mathcal{S}=1 / 2 \rightarrow \otimes \mathrm{E}_{1 / 2 g}\right)$ yields the subsequent splittings:

$$
{ }^{2} \mathrm{E}_{g} \otimes \mathrm{E}_{1 / 2 g}=\mathrm{F}_{3 / 2 g} .
$$

Hence, the combined ground state is ${ }^{2} \mathrm{E}_{g}\left[\mathrm{~F}_{3 / 2 g}\right]\left(\mathrm{O}_{h}\right)$.

The intermediate state configuration $1 \mathrm{~s}^{1} 3 \mathrm{~d}^{8}$ is still the same as in the high spin case; however, the higher crystal field results in different energies for the intermediate state energy levels. In the low spin case, the initial state being a spin doublet, the spin-conserving 1s XAS only reaches spin doublet intermediate state terms (Table 3), which correspond to the crystal field terms issued from ${ }^{2} \mathrm{~S},{ }^{2} \mathrm{P},{ }^{2} \mathrm{D},{ }^{2} \mathrm{~F}$, and ${ }^{2} \mathrm{G}$ and ${ }^{2} \mathrm{~A}_{1 g},{ }^{2} \mathrm{~A}_{2 g}$, ${ }^{2} \mathrm{E}_{g},{ }^{2} \mathrm{~T}_{1 g}$, and ${ }^{2} \mathrm{~T}_{2 g}$. The quadrupole 1s XAS starts from the low spin ground state term ${ }^{2} \mathrm{E}_{g}$ revealing the reachable terms:

$$
\begin{aligned}
& { }^{2} \mathrm{E}_{g} \stackrel{\otimes \mathrm{T}_{2 g}}{\longrightarrow}{ }^{2} \mathrm{~T}_{1 g} \oplus{ }^{2} \mathrm{~T}_{2 g}, \\
& \underbrace{{ }^{2} \mathrm{E}_{g}}_{\text {GS }} \stackrel{{ }_{1 s} \mathrm{XAS}}{\longrightarrow} \underbrace{2 \mathrm{~A}_{1 g} \oplus^{2} \mathrm{~A}_{2 g} \oplus^{2} \mathrm{E}_{g}}_{\text {Reachable IS }} \text {. }
\end{aligned}
$$




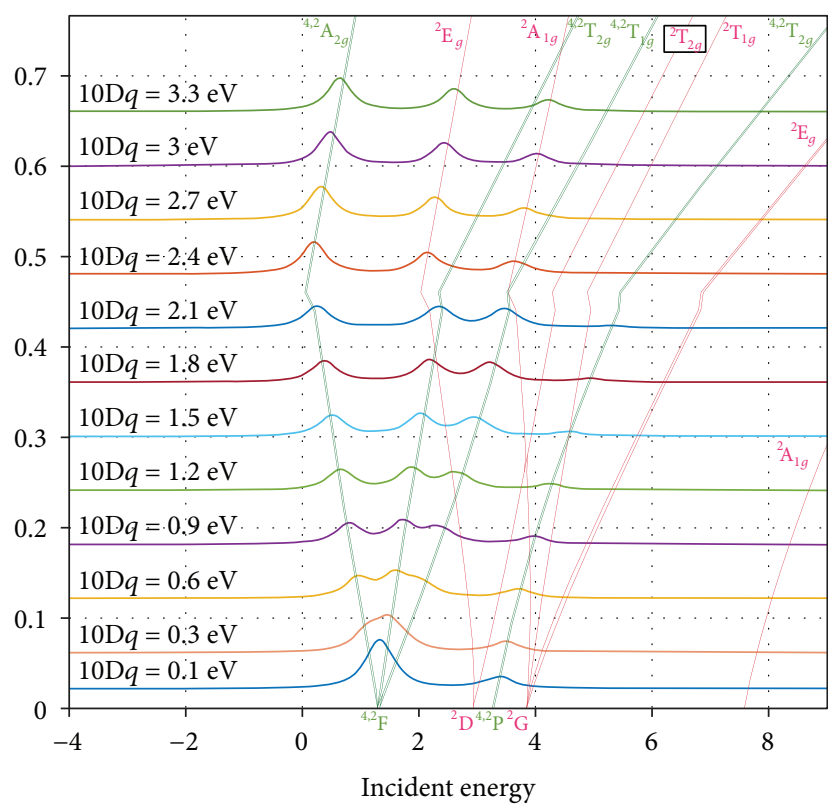

FIGURE 53: 1s XAS projections of a calculated 1s2p RIXS spectrum for $\mathrm{Co}^{2+}\left(3 \mathrm{~d}^{7}\right)$ with ELD. It confirms that in high spin only the quartet terms ${ }^{4,2} \mathrm{~F}$ and ${ }^{4,2} \mathrm{P}$ contribute to the $1 \mathrm{~s}$ XAS. The high spin to low spin transition appears at approximately $10 \mathrm{D} q \approx 2.2 \mathrm{eV}$.

Thus, according to the quadrupole selection rules, from the ${ }^{2} \mathrm{E}_{g}$ ground state, the 1s XAS transitions can reach all existing spin doublet IRREPs in the intermediate state. However, because in the low spin initial state all $t_{2 g}$ orbitals are occupied, 1s XAS transitions can only go into the $e_{g}$ orbitals via the quadrupole $\mathrm{E}_{g}$ IRREP. The transitions into the $t_{2 g}$ orbitals with the quadrupole $\mathrm{T}_{2 g}$ IRREP are less probable as they require a two-electron transition. In other words, the matrix elements of the quadrupole $\mathrm{E}_{g}$ IRREP are larger than those of the quadrupole $\mathrm{T}_{2 g}$ IRREP. The resulting 1s XAS projections for the low spin case are shown in Figure 55.

For the 1p XES decays, the inclusion of $3 \mathrm{~d}$ SOC $(\mathcal{S}=$ $\left.1 / 2 \rightarrow \otimes \mathrm{E}_{1 / 2 g}\right)$ is again required, leading to the following splittings of the five reachable terms in the intermediate state:

$$
\begin{aligned}
& { }^{2} \mathrm{~A}_{1 g} \otimes \mathrm{E}_{1 / 2 g}=\mathrm{E}_{1 / 2 g}, \\
& { }^{2} \mathrm{~A}_{2 g} \otimes \mathrm{E}_{12 g}=\mathrm{E}_{5 / 2 g}, \\
& { }^{2} \mathrm{E}_{g} \otimes \mathrm{E}_{1 / 2 g}=\mathrm{F}_{3 / 2 g}, \\
& { }^{2} \mathrm{~T}_{1 g} \otimes \mathrm{E}_{1 / 2 g}=\mathrm{E}_{1 / 2 g} \oplus \mathrm{F}_{3 / 2 g}, \\
& { }^{2} \mathrm{~T}_{2 g} \otimes \mathrm{E}_{1 / 2 g}=\mathrm{E}_{5 / 2 g} \oplus \mathrm{F}_{3 / 2 g} .
\end{aligned}
$$

Hence, the $2 p$ XES decays in $1 s 2 p$ RIXS can occur from any of these three total angular momentum IRREPs $\mathrm{E}_{1 / 2 g}$, $\mathrm{E}_{5 / 2 g}$, or $\mathrm{F}_{3 / 2 g}$.

The final state electron configuration $1 s^{2} 2 \mathrm{p}^{5} 3 \mathrm{~d}^{8}$ is again identical to the high spin case regardless of the crystal field effects, offering the three total angular momentum IRREPs $\mathrm{E}_{1 / 2 u}, \mathrm{E}_{5 / 2 u}$, and $\mathrm{F}_{3 / 2 u}$. For the $2 \mathrm{p}$ XES decaying from the populated intermediate state terms, using the $\mathrm{T}_{1 u}$ IRREP (Table 4) again reveals that all three final state IRREPs are also reachable. Furthermore, in 2p XAS, starting from the $\mathrm{F}_{3 / 2 g}$ ground state symmetry, also, all three final state IRREPs are reachable (Table 4 ).

The results according to the selection rules as discussed above are again summarised in a combined term scheme as displayed in Figure 52.

Again, we conclude with an example calculation for a $\mathrm{Co}^{2+}$ ion $\left(3 \mathrm{~d}^{7}\right.$ ground state configuration) with $10 \mathrm{Dq}=3.0 \mathrm{e}$ $\mathrm{V}$ reflecting the low spin case as shown in Figure 56.

It is noteworthy that the quadrupole IRREP $\mathrm{T}_{2 g}$ here is very weak $\left(10^{-3}\right)$ with respect to the $\mathrm{E}_{g}$ IRREP. This can again be explained with the very small matrix elements for the two-electron transition that is required to create a hole in the $t_{2 g}$ orbitals, because the quadrupole IRREP $\mathrm{T}_{2 g}$ populates the intermediate state symmetries $\mathrm{T}_{1 g}$ and $\mathrm{T}_{2 g}$, which both require a hole in the $t_{2 g}$ level.

This is also confirmed by the total RIXS map (Figure 56(a), right) showing that the overall result is dominated by the transitions into the three intermediate state symmetries ${ }^{2} \mathrm{~A}_{2 g},{ }^{2} \mathrm{E}_{g}$, and ${ }^{2} \mathrm{~A}_{1 g}$, while the ${ }^{2} \mathrm{~T}_{1 g}$ and ${ }^{2} \mathrm{~T}_{2 g}$ intermediate state symmetries are negligible due to the reason given above.

However, as predicted by the selection rules for the quadrupole IRREPS, $\mathrm{T}_{2 g}$ and $\mathrm{E}_{g}$ as shown in Figure 56 confirm the predicted reachable terms as found in expression (79).

5.8. $1 s 2 p$ RIXS Experiments of $3 \mathrm{~d}^{7}$ Systems. A $300 \mathrm{meV}$ resolution 1s2p RIXS experiment on $\mathrm{CoO}$ was published by 

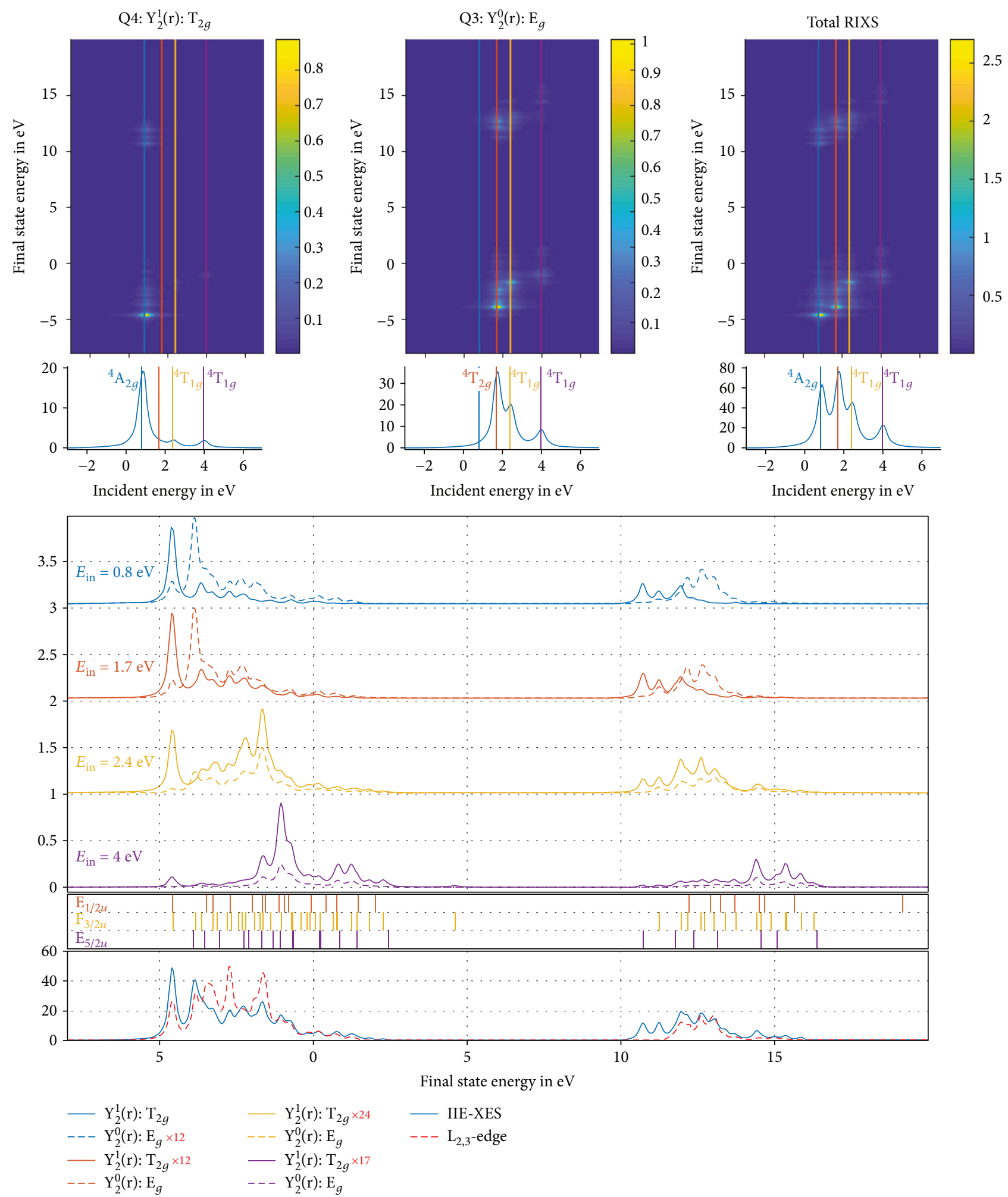

Figure 54: Calculated 1s2p RIXS spectrum for $\mathrm{Co}^{2+}\left(3 \mathrm{~d}^{7}\right)$ for $10 \mathrm{D} q=0.9 \mathrm{eV}$ (high spin).

Kurian et al. [50]. The increased resolution revealed new details in the pre-edge fine structure, and it was possible to study the effect of interference effects on the 1 s $2 p$ RIXS plane.

\section{Concluding Remarks}

We have presented first three didactic cases, being the $\mathrm{Cu}^{2+}\left(3 \mathrm{~d}^{9}\right), \mathrm{Ti}^{4+}\left(3 \mathrm{~d}^{0}\right)$, and $\mathrm{Ni}^{2+}\left(3 \mathrm{~d}^{8}\right)$, respectively, for 


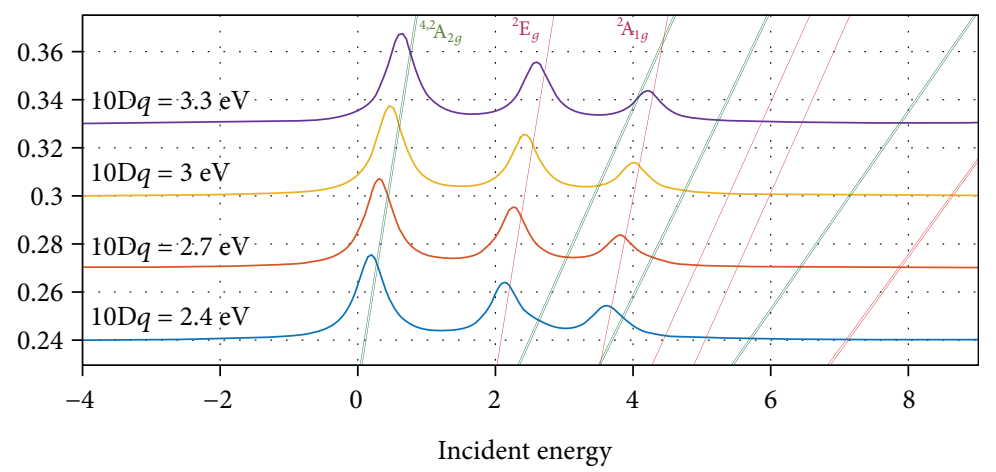

Figure 55: 1s XAS projections of the calculated 1s2p RIXS maps for a $\mathrm{Co}^{2+}$ ion $\left(3 \mathrm{~d}^{7}\right)$ with $10 \mathrm{D} q=2.4 \mathrm{eV}$ to $3.3 \mathrm{eV}$ (low spin only).

which detailed descriptions were given. For those cases, we included also an atomic calculation without a crystal field to illustrate the effect an octahedral crystal field has. The didactic cases were chosen as these cases have a low number of microstates involved (e.g., having an almost full or almost empty $3 \mathrm{~d}$ shell). Though the effort would be significantly larger, when needed, one can further separate the 2p XES transitions for the more complex cases discussed in Section 5. For example, calculations with an unrealistic low natural broadening (e.g., $1-2$ $\mathrm{meV}$ ) would enable to do a detailed fingerprint comparison with the stick spectra allowing to assign the peaks.

Altogether, we illustrated the effects of an octahedral crystal field and the selection rules for an example ion for every $3 \mathrm{~d}^{N}$ ground state case (with $N=\{0, \ldots, 9\}$ ). Here, we provide a few selected examples of calculations. For an even better illustration, one can for example combine the calculated RIXS maps or combine many slices into short movie clips where one or more parameters (e.g., $10 \mathrm{D} q$ or SOC) are scaled. For the crystal field parameter $10 \mathrm{D} q$, such scaling would relate to the corresponding Tanabe-Sugano diagram which is also shown throughout this paper for the 1s XAS projections.

Another important aspect is the mixing of the states induced by spin-orbit or exchange interactions, as well as the crystal field. Although we have assigned labels to many states involved in the transitions, those labels represent only the dominant contribution to each state. In reality, most states are not pure in their nature as it was also mentioned for several cases throughout this paper. As can be seen for example in the $\mathrm{Cr}^{3+}, \mathrm{Mn}^{3+}$, or $\mathrm{Fe}^{3+}$ cases, the stick spectra show a very dense distribution of states. To give a detailed insight into the structure of the spectra, we have used a rather small Lorentzian broadening, while any Gaussian broadening was omitted for all calculations. Hence, when a realistic natural broadening is assumed and an experimental broadening is also considered, one can hardly separate the transitions visible in one of the complex spectra. Due to the often very small splittings in many cases, the states appear as a band rather than discrete states. The difference between the final states probed in 1 s 2 p RIXS versus 2 p XAS is emphasised for each $3 \mathrm{~d}^{N}$ case.
The computational effort is for the complex cases quite time-consuming, especially when aiming for highresolution spectra which are needed when the broadenings are small. However, this effort is justified as it helps to get a better understanding of RIXS spectra and they can in some cases be also useful to determine for example the spin state (high spin versus low spin).

With increasing computational power being available in the future, also Monte Carlo-like calculations can become a favourable approach, as one can for example calculate "all RIXS spectra" for a sufficient section of the parameter space and then select the best fit. However, it is safe to assume that for most ions such calculations will yield multiple results making the combination with additional information, especially for more complex cases, necessary. Although we show the importance of the multielectronic excitations in the $1 \mathrm{~s} 2 \mathrm{p}$ RIXS, the emerging first-principle estimation of the crystal field parameters will open new opportunities for the modelling of experimental data.

For each case of the $3 \mathrm{~d}^{N}$ series, we have discussed the $1 s 2 p$ RIXS crystal field calculation in the light of the available experimental data. With few exceptions, 1s $2 p$ RIXS experiments are to date performed with an experimental resolution of $500 \mathrm{meV}$ or more. This resolution does not allow a competitive comparison with $2 \mathrm{p}$ XAS regarding the details of the electronic structure parameters as discussed here. The constant improvements of the experimental resolution in hard X-ray spectroscopy enable to acquire a better experimental spectrum where natural broadenings are approached as the ultimate limit. An overall experimental resolution of $200 \mathrm{meV}$ would be necessary to improve upon the determination of the parameters. Tetravalent systems are dominated by nonlocal dipole transitions that overwhelm the quadrupole pre-edges. This makes the analysis of $1 \mathrm{~s} 2 \mathrm{p}$ RIXS complicated. The best systems to study the details of the $1 \mathrm{~s} 2 \mathrm{p}$ RIXS resonances are ionic divalent systems.

\section{Conflicts of Interest}

The authors declare that there is no conflict of interest regarding the publication of this article. 

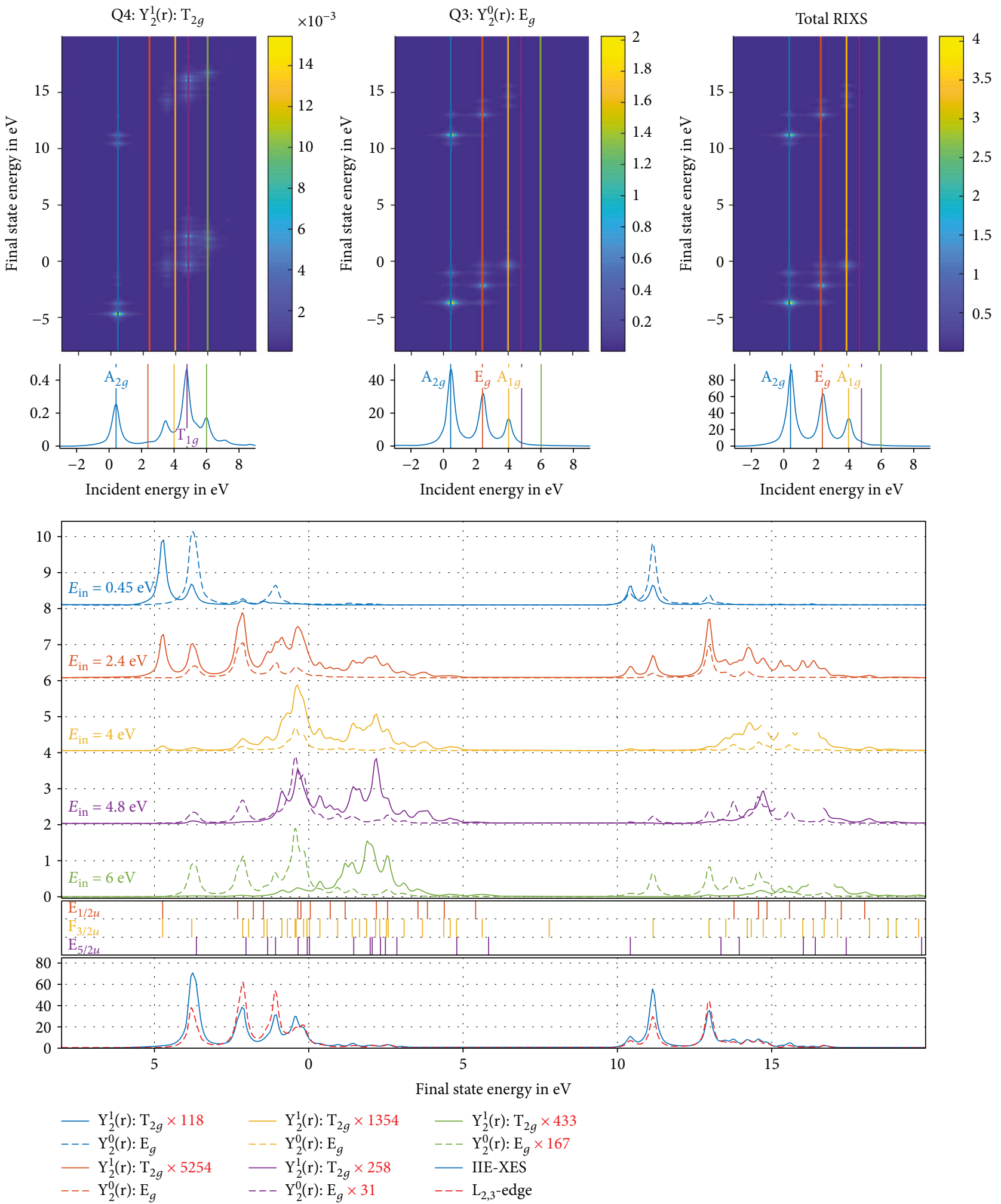

FIgURE 56: Calculated 1s2p RIXS spectrum for $\mathrm{Co}^{2+}\left(3 \mathrm{~d}^{7}\right)$ for $10 \mathrm{D} q=3.0 \mathrm{eV}$ (low spin).

\section{Acknowledgments}

The authors wish to acknowledge Maurits Harverkort (Heidelberg) and Robert Green (UBC) for their support on the Quanty software and answering all questions always quickly in a very detailed manner. For financial support, they acknowledge the European Research Council (ERC advanced Grant 340279). 


\section{References}

[1] A. Bagger, T. Haarman, A. Puig Molina et al., "1s2p resonant inelastic X-ray scattering combined dipole and quadrupole analysis method," Journal of Synchrotron Radiation, vol. 24, pp. 296-301, 2017.

[2] H. Hayashi, Y. Udagawa, W. A. Caliebe, and C. C. Kao, "Hidden electronic state of $\mathrm{CuO}$ revealed by resonant inelastic x-ray scattering," Physical Review B, vol. 66, no. 3, article 033105, 2002.

[3] D. Cabaret, Y. Joly, H. Renevier, and C. R. Natoli, "Preedge structure analysis of $\mathrm{Ti} K$-edge polarized X-ray absorption spectra in $\mathrm{TiO} 2$ by full-potential XANES calculations," Journal of Synchrotron Radiation, vol. 6, no. 3, pp. 258-260, 1999.

[4] D. Cabaret, A. Bordage, A. Juhin, M. Arfaoui, and E. Gaudry, "First-principles calculations of X-ray absorption spectra at the $K$-edge of $3 d$ transition metals: an electronic structure analysis of the pre-edge," Physical Chemistry Chemical Physics, vol. 12, no. 21, pp. 5619-5633, 2010.

[5] M. O. J. Y. Hunault, W. Khan, J. Minár et al., "Local vs nonlocal states in $\mathrm{FeTiO}_{3}$ probed with 1s2pRIXS: implications for photochemistry," Inorganic Chemistry, vol. 56, no. 18, pp. 10882-10892, 2017.

[6] A. Juhin, F. M. F. de Groot, G. Vankó, M. Calandra, and C. Brouder, "Angular dependence of core hole screening in $\mathrm{LiCoO}_{2}$ : a DFT $+U$ calculation of the oxygen and cobalt $K$-edge x-ray absorption spectra," Physical Review B, vol. 81, no. 11, article 115115, 2010.

[7] G. Vankó, F. M. F. de Groot, S. Huotari, R. J. Cava, T. Lorenz, and M. Reuther, "Intersite $4 \mathrm{p}-3 \mathrm{~d}$ hybridization in cobalt oxides: a resonant x-ray emission spectroscopy study," 2008, https://arxiv.org/abs/0802.2744.

[8] T. E. Westre, P. Kennepohl, J. G. DeWitt, B. Hedman, K. O. Hodgson, and E. I. Solomon, "A multiplet analysis of Fe Kedge $1 \mathrm{~s} \rightarrow 3 \mathrm{~d}$ pre-edge features of iron complexes," Journal of the American Chemical Society, vol. 119, no. 27, pp. 62976314, 1997.

[9] P. Zimmermann, N. Bouldi, M. O. J. Y. Hunault et al., "1s2p resonant inelastic X-ray scattering magnetic circular dichroism as a probe for the local and non-local orbitals in $\mathrm{CrO}_{2}$," Journal of Electron Spectroscopy and Related Phenomena, vol. 222, pp. 74-87, 2018.

[10] S. DeBeer George, P. Brant, and E. I. Solomon, "Metal and ligand K-edge XAS of organotitanium complexes: metal $4 \mathrm{p}$ and $3 \mathrm{~d}$ contributions to pre-edge intensity and their contributions to bonding," Journal of the American Chemical Society, vol. 127, no. 2, pp. 667-674, 2005.

[11] T. Kroll, M. Lundberg, and E. I. Solomon, "X-ray absorption and RIXS on coordination complexes," in X-Ray Absorption and X-Ray Emission Spectroscopy: Theory and Applications, John Wiley \& Sons, Ltd, Chichester, UK, 2016.

[12] V. Vercamer, M. O. J. Y. Hunault, G. Lelong et al., "Calculation of optical and $K$ pre-edge absorption spectra for ferrous iron of distorted sites in oxide crystals," Physical Review B, vol. 94, no. 24 , article $245115,2016$.

[13] F. de Groot and A. Kotani, Core Level Spectroscopy of Solids, Advances in condensed matter science, CRC Press, Boca Raton, FL, USA, 2008.

[14] P. Glatzel and U. Bergmann, "High resolution 1s core hole Xray spectroscopy in $3 \mathrm{~d}$ transition metal complexes-electronic and structural information," Coordination Chemistry Reviews, vol. 249, no. 1-2, pp. 65-95, 2005.

[15] J. P. Rueff and A. Shukla, "A RIXS cookbook: five recipes for successful RIXS applications," Journal of Electron Spectroscopy and Related Phenomena, vol. 188, pp. 10-16, 2013.

[16] H. Ågren and F. Gel'mukhanov, "Kramers-Heisenberg and Weisskopf-Wigner descriptions of resonant X-ray Raman scattering," Journal of Electron Spectroscopy and Related Phenomena, vol. 110-111, pp. 153-178, 2000.

[17] F. M. F. de Groot, P. Glatzel, U. Bergmann et al., "1s2p resonant inelastic X-ray scattering of iron oxides," The Journal of Physical Chemistry B, vol. 109, no. 44, pp. 20751-20762, 2005.

[18] P. Glatzel, M. Sikora, and M. Fernández-García, "Resonant Xray spectroscopy to study $\mathrm{K}$ absorption pre-edges in 3d transition metal compounds," The European Physical Journal Special Topics, vol. 169, no. 1, pp. 207-214, 2009.

[19] J.-E. Rubensson, J. Luning, S. Eisebitt, and W. Eberhardt, "It's always a one-step process," Applied Physics A, vol. 65, no. 2, pp. 91-96, 1997.

[20] L. J. P. Ament, M. van Veenendaal, T. P. Devereaux, J. P. Hill, and J. van den Brink, "Resonant inelastic x-ray scattering studies of elementary excitations," Reviews of Modern Physics, vol. 83, no. 2, pp. 705-767, 2011.

[21] F. M. de Groot, "Multiplet effects in X-ray spectroscopy," Coordination Chemistry Reviews, vol. 249, no. 1-2, pp. 31-63, 2005.

[22] E. U. Condon and G. H. Shortley, The Theory of Atomic Spectra, Cambridge Press, Cambridge, UK, 1959.

[23] R. Xu and Z. Dai, "Alternative mathematical technique to determine LS spectral terms," Journal of Physics B: Atomic, Molecular and Optical Physics, vol. 39, no. 16, pp. 32213239, 2006.

[24] C. Brouder, "Angular dependence of x-ray absorption spectra," Journal of Physics: Condensed Matter, vol. 2, no. 3, pp. 701-738, 1990.

[25] A. Juhin, C. Brouder, and F. M. de Groot, "Angular dependence of resonant inelastic x-ray scattering: a spherical tensor expansion," Open Physics, vol. 12, no. 5, pp. 323-340, 2014.

[26] C. Brouder, A. Juhin, A. Bordage, and M.-A. Arrio, "Site symmetry and crystal symmetry: a spherical tensor analysis," Journal of Physics: Condensed Matter, vol. 20, no. 45, article 455205, 2008.

[27] B. T. Thole, R. D. Cowan, G. A. Sawatzky, J. Fink, and J. C. Fuggle, "New probe for the groundstate electronic structure of narrow-band and impurity systems," Physical Review B, vol. 31, no. 10, pp. 6856-6858, 1985.

[28] P. H. Butler, Point Group Symmetry Applications: Methods and Tables, Plenum Press, New York, NY, USA, 1981.

[29] R. D. Cowan, The Theory of Atomic Structure and Spectra, University of California Press, Berkeley, CA, USA, 1981.

[30] M. W. Haverkort, "Quanty for core level spectroscopy - excitons, resonances and band excitations in time and frequency domain," Journal of Physics: Conference Series, vol. 712, no. 1, article 012001, 2016.

[31] M. W. Haverkort, M. Zwierzycki, and O. K. Andersen, "Multiplet ligand-field theory using Wannier orbitals," Physical Review B, vol. 85, no. 16, pp. 165113-165120, 2012.

[32] P. Zimmermann, R. J. Green, M. W. Haverkort, and F. M. F. de Groot, "Quanty4RIXS: a program for crystal field multiplet 
calculations of RIXS and RIXS-MCD spectra using Quanty," Journal of Synchrotron Radiation, vol. 25, no. 3, pp. 899-905, 2018.

[33] E. Stavitski and F. M. F. de Groot, "The CTM4XAS program for EELS and XAS spectral shape analysis of transition metal L edges," Micron, vol. 41, no. 7, pp. 687-694, 2010.

[34] M. W. Haverkort, "Theory of resonant inelastic X-ray scattering by collective magnetic excitations," Physical Review Letters, vol. 105, no. 16, article 167404, 2010.

[35] F. M. F. de Groot, J. C. Fuggle, B. T. Thole, and G. A. Sawatzky, " $L_{2}, 3 \mathrm{X}$-ray-absorption edges of $d^{0}$ compounds: $\mathrm{K}^{+}, \mathrm{Ca}^{2+}, \mathrm{Sc}^{3+}$ and $\mathrm{Ti}^{4+}$ in $\mathrm{O}_{h}$ (octahedral) symmetry," Physical Review $B$, vol. 41, no. 2, pp. 928-937, 1990.

[36] F. M. F. de Groot, "X-ray absorption of transition metal oxides: an overview of the theoretical approaches," Journal of Electron Spectroscopy and Related Phenomena, vol. 62, no. 1-2, pp. 111130, 1993.

[37] T. Kroll, E. I. Solomon, and F. M. F. de Groot, "Final-state projection method in charge-transfer multiplet calculations: an analysis of Ti L-edge absorption spectra," The Journal of Physical Chemistry B, vol. 119, no. 43, pp. 13852-13858, 2015.

[38] P. Glatzel, T.-C. Weng, K. Kvashnina et al., "Reflections on hard X-ray photon-in/photon-out spectroscopy for electronic structure studies," Journal of Electron Spectroscopy and Related Phenomena, vol. 188, pp. 17-25, 2013.

[39] J. J. Kas, J. J. Rehr, J. A. Soininen, and P. Glatzel, "Real-space Green's function approach to resonant inelastic x-ray scattering," Physical Review B, vol. 83, no. 23, article 235114, 2011.

[40] P. Glatzel, U. Bergmann, W. Gu et al., "Electronic structure of $\mathrm{Ni}$ complexes by X-ray resonance Raman spectroscopy (resonant inelastic X-ray scattering)," Journal of the American Chemical Society, vol. 124, no. 33, pp. 9668-9669, 2002.

[41] A. Bordage, Propriétés Spectroscopiques et Structure Électronique du Vanadium dans des Matériaux Complexes: Implications Géologiques et Technologiques, [Ph.D. thesis], Universite Pierre et Marie Curie, Paris, 2009.

[42] J. Frommer, M. Nachtegaal, I. Czekaj, T.-C. Weng, and R. Kretzschmar, "X-ray absorption and emission spectroscopy of $\mathrm{Cr}^{\mathrm{III}}$ (Hydr)oxides: analysis of the K-pre-edge region," The Journal of Physical Chemistry A, vol. 113, no. 44, pp. 1217112178, 2009.

[43] P. Glatzel, U. Bergmann, J. Yano et al., "The electronic structure of $\mathrm{Mn}$ in oxides, coordination complexes, and the oxygen-evolving complex of photosystem II studied by resonant inelastic X-ray scattering," Journal of the American Chemical Society, vol. 126, no. 32, pp. 9946-9959, 2004.

[44] V. Vercamer, Spectroscopic and Structural Properties of Iron in Silicate Glasses, [Ph.D. thesis], Université Pierre et Marie Curie - Paris VI, Paris, France, 2016, https://tel.archivesouvertes.fr/tel-01458771/.

[45] M. Lundberg, T. Kroll, S. DeBeer et al., "Metal-ligand covalency of iron complexes from high-resolution resonant inelastic X-ray scattering," Journal of the American Chemical Society, vol. 135, no. 45, pp. 17121-17134, 2013.

[46] J. P. Rueff, L. Journel, P. E. Petit, and F. Farges, "Fe K pre-edges as revealed by resonant Xray emission," Physical Review B, vol. 69, no. 23, article 235107, 2004.

[47] M. Guo, E. Källman, L. K. Sørensen, M. G. Delcey, R. V. Pinjari, and M. Lundberg, "Molecular orbital simulations of metal $1 \mathrm{~s} 2 \mathrm{p}$ resonant inelastic X-ray scattering," The Journal of Physical Chemistry A, vol. 120, no. 29, pp. 5848-5855, 2016.

[48] N. Leidel, P. Chernev, K. G. V. Havelius, L. Schwartz, S. Ott, and M. Haumann, "Electronic structure of an [FeFe] Hydrogenase model complex in solution revealed by X-ray absorption spectroscopy using narrow-band emission detection," Journal of the American Chemical Society, vol. 134, no. 34, pp. 14142-14157, 2012.

[49] G. Vankó, A. Bordage, M. Pápai et al., "Detailed characterization of a nanosecond-lived excited state: X-ray and theoretical investigation of the quintet state in photoexcited $\left[\mathrm{Fe}(\text { terpy })_{2}\right]^{2+}$," The Journal of Physical Chemistry C, vol. 119, no. 11, pp. 5888-5902, 2015.

[50] R. Kurian, M. M. van Schooneveld, N. Zoltán, G. Vankó, and F. M. F. de Groot, "Temperature-dependent $1 \mathrm{~s} 2 \mathrm{p}$ resonant inelastic X-ray scattering of CoO," Journal of Physical Chemistry C, vol. 117, no. 6, pp. 2976-2981, 2013. 

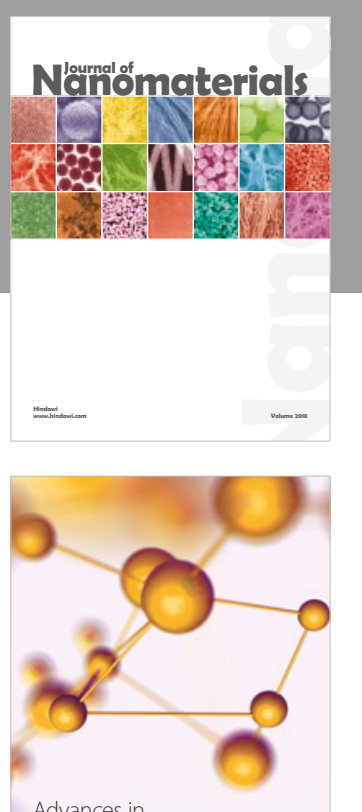

Physical Chemistry
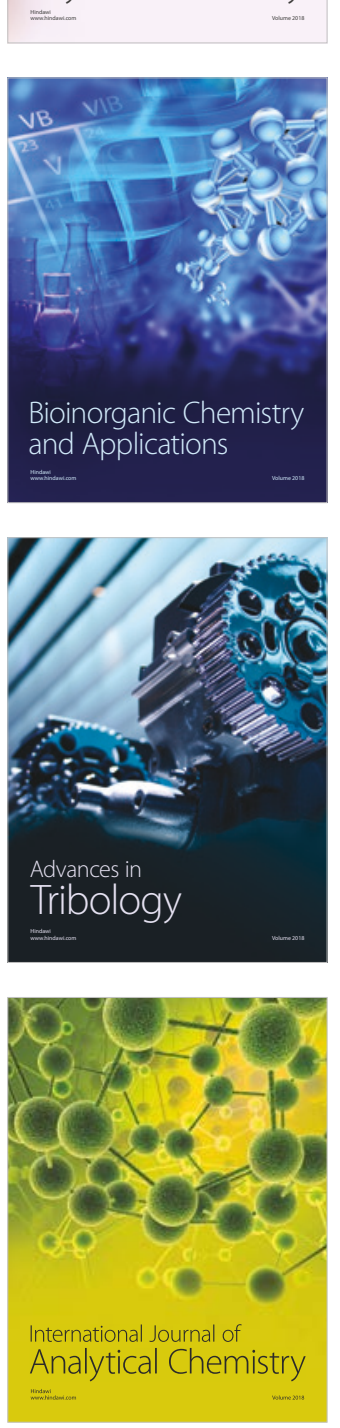

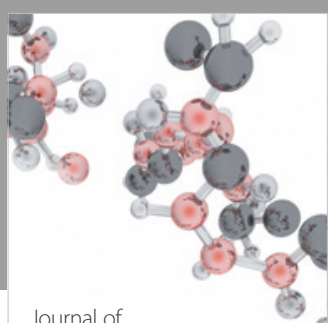

Analytical Methods

in Chemistry

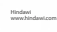

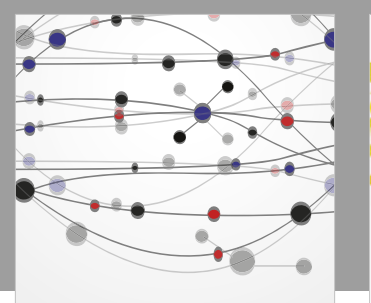

The Scientific World Journal

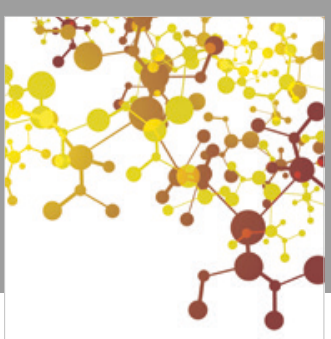

Journal of

Applied Chemistry
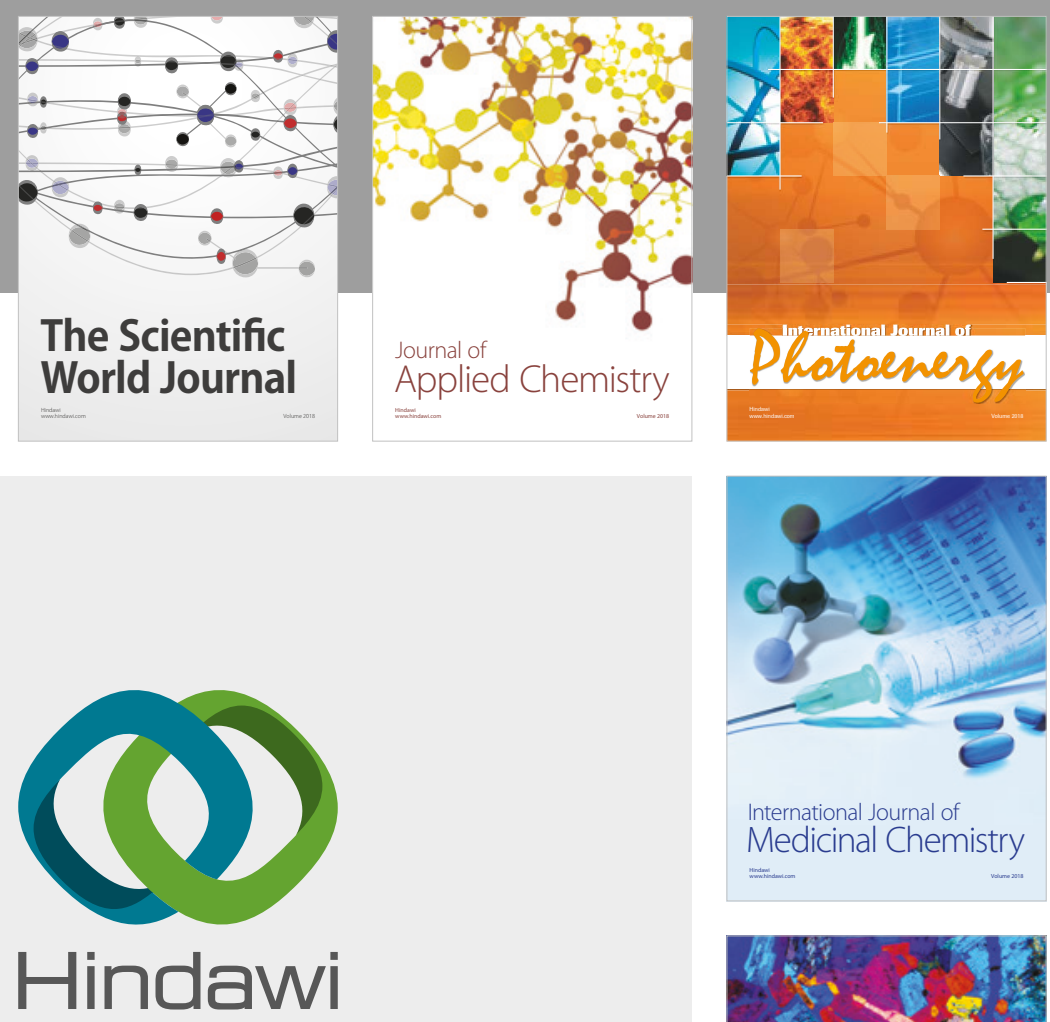

Submit your manuscripts at

www.hindawi.com
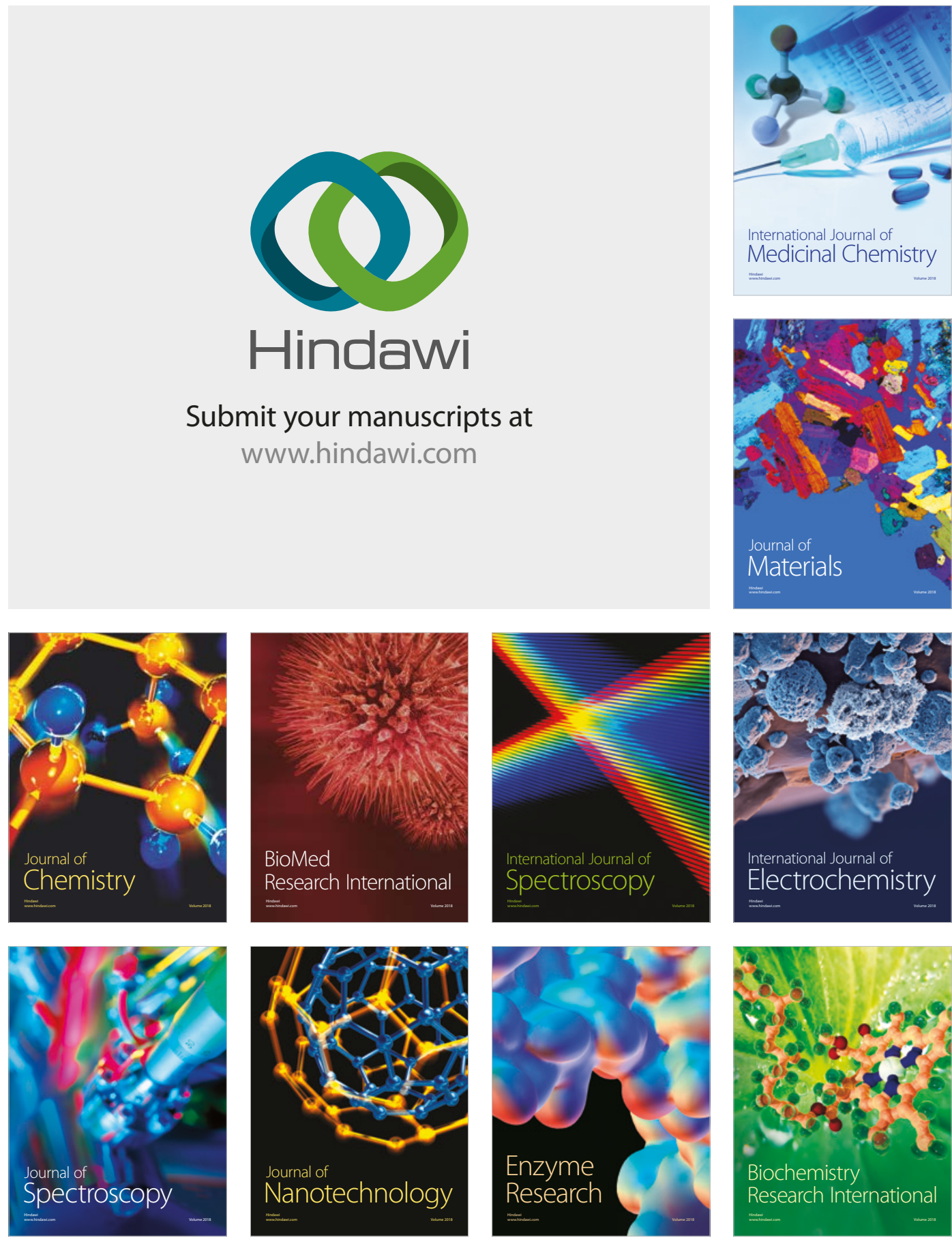
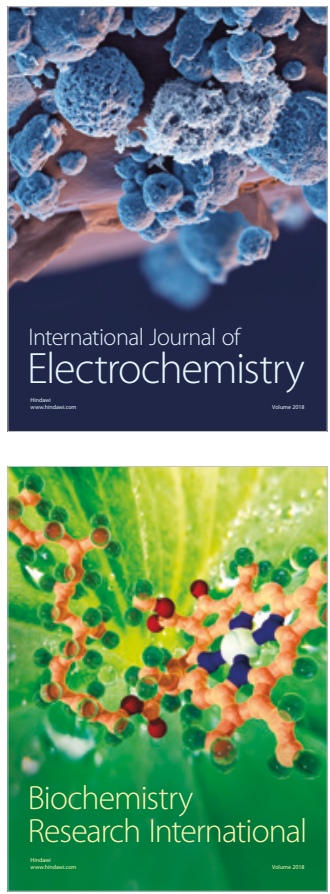